Research paper

\title{
Geologic implications of gas hydrates in the offshore of India: Results of the National Gas Hydrate Program Expedition 01
}

\author{
Timothy S. Collett a, *, Ray Boswell b , James R. Cochran c, Pushpendra Kumar ${ }^{\text {d }}$, \\ Malcolm Lall ${ }^{\mathrm{e}}$, Aninda Mazumdar ${ }^{\mathrm{f}}$, Mangipudi Venkata Ramana ${ }^{\mathrm{g}}$, \\ Tammisetti Ramprasad ${ }^{\mathrm{f}}$, Michael Riedel ${ }^{\mathrm{h}}$, Kalachand Sain ${ }^{\mathrm{i}}$, Arun Vasant Sathe ${ }^{\mathrm{j}}$, \\ Krishna Vishwanath ${ }^{\mathrm{e}}$, NGHP Expedition 01 Scientific Party \\ ${ }^{a}$ U.S. Geological Survey, Denver Federal Center, MS-939, Box 25046, Denver, CO 80225, USA \\ ${ }^{\mathrm{b}}$ U.S. Department of Energy, National Energy Technology Laboratory, 626 Cochran's Mill Rd., Pittsburgh, PA 15236, USA \\ ${ }^{\mathrm{c}}$ Lamont Doherty Earth Observatory, Columbia University, Palisades, NY 10964, USA \\ d Oil and Natural Gas Corporation Ltd., Institute of Engineering and Ocean Technology, ONGC Complex, Phase II, Panvel 410221, Navi Mumbai, India \\ e Directorate General of Hydrocarbons, Plot No 2, Sector 73, Noida, India \\ ${ }^{\mathrm{f}}$ CSIR-National Institute of Oceanography, Donapaula, Goa 403004, India \\ ${ }^{\mathrm{g}}$ Mauritius Oceanography Institute, Victoria Avenue, 230427 4434, Mauritius

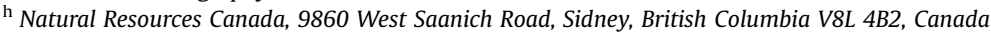 \\ ${ }^{\mathrm{i}}$ CSIR-National Geophysical Research Institute, Uppal Road, Hyderabad 500007, India \\ ${ }^{j}$ Oil and Natural Gas Corporation Ltd., KDM Inst. of Petroleum Exploration, 9 Kaulagarh Road, Dehradun 248195, Uttaranchal, India
}

\section{A R T I C L E I N F O}

\section{Article history:}

Received 19 July 2014

Accepted 22 July 2014

Available online 4 September 2014

\section{Keywords:}

Gas hydrate

Gas

Resources

India

Krishna-Godavari Basin

Mahanadi Basin

Andaman Sea

Kerala-Konkan Basin

\section{A B S T R A C T}

The Indian National Gas Hydrate Program Expedition 01 (NGHP-01) is designed to study the occurrence of gas hydrate along the passive continental margin of the Indian Peninsula and in the Andaman convergent margin, with special emphasis on understanding the geologic and geochemical controls on the occurrence of gas hydrate in these two diverse settings. The NGHP-01 expedition established the presence of gas hydrates in the Krishna-Godavari and Mahanadi Basins, and the Andaman Sea. The expedition discovered in the Krishna-Godavari Basin one of the thickest gas hydrate accumulations ever documented, in the Andaman Sea one of the thickest and deepest gas hydrate stability zones in the world, and established the existence of a fully developed gas hydrate petroleum system in all three basins.

The primary goal of NGHP-01 was to conduct scientific ocean drilling/coring, logging, and analytical activities to assess the geologic occurrence, regional context, and characteristics of gas hydrate deposits along the continental margins of India. This was done in order to meet the long-term goal of exploiting gas hydrate as a potential energy resource in a cost effective and safe manner. During its 113.5-day voyage, the D/V JOIDES Resolution cored and/or drilled 39 holes at 21 sites ( 1 site in Kerala-Konkan, 15 sites in Krishna-Godavari, 4 sites in Mahanadi, and 1 site in the Andaman deep offshore area), penetrated more than $9250 \mathrm{~m}$ of sedimentary section, and recovered nearly $2850 \mathrm{~m}$ of core. Twelve holes were logged with logging-while-drilling (LWD) tools and an additional 13 holes were wireline logged. The science team utilized extensive on-board laboratory facilities to examine and prepare preliminary reports on the physical properties, geochemistry, and sedimentology of all the data collected prior to the end of the expedition. Samples were also analyzed in additional post-expedition shore-based studies conducted in leading laboratories around the world.

One of the specific objectives of this expedition was to test gas hydrate formation models and constrain model parameters, especially those that account for the formation of concentrated gas hydrate accumulations. The necessary data for characterizing the occurrence of in situ gas hydrate, such as interstitial water chlorinities, core-derived gas chemistry, physical and sedimentological properties, thermal images of the recovered cores, and downhole measured logging data (LWD and/or conventional wireline log data), were obtained from most of the drill sites established during NGHP-01. Almost all of the drill sites yielded evidence for the occurrence of gas hydrate; however, the inferred in situ

\footnotetext{
* Corresponding author.

E-mail address: tcollett@usgs.gov (T.S. Collett).
} 
concentration of gas hydrate varied substantially from site to site. For the most part, the interpretation of downhole logging data, core thermal images, interstitial water analyses, and pressure core images from the sites drilled during NGHP-01 indicate that the occurrence of concentrated gas hydrate is mostly associated with the presence of fractures in the sediments, and in some limited cases, by coarser grained (mostly sand-rich) sediments.

Published by Elsevier Ltd.

\section{Editor's note}

This report is part one of a two-report series that summarizes the results of the Indian National Gas Hydrate Program Expedition 01 (NGHP-01). This first report (Collett et al., this issue) in this series summarizes the overall operational and scientific accomplishments of NGHP-01, while the second report reviews the occurrence and geologic controls on gas hydrates in the Krishna-Godavari Basin, the Mahanadi Basin, the Andaman Sea, and the Kerala-Konkan Basin (Kumar et al., this issue).

\section{Introduction}

Gas hydrate is a naturally occurring "ice-like" combination of natural gas and water that has the potential to serve as an immense resource of natural gas from the world's oceans and polar regions. Gas hydrate recovery is a scientific and a technical challenge and much remains to be learned about the geologic, engineering, and economic factors controlling the ultimate energy resource potential of gas hydrate. The estimate of natural gas contained in the world's gas hydrate accumulations is enormous, but these estimates are speculative and range over three orders of magnitude from about 1 to 100 trillion cubic meters of gas (reviewed by Boswell and Collett, 2011). Gas recovery from gas hydrate is made more difficult because the gas is in a solid form and because gas hydrate commonly occurs in remote Arctic and deep marine environments. Proposed methods of gas recovery from gas hydrate include disassociating or "melting" in situ gas hydrate by heating the reservoir beyond the temperature of gas hydrate formation, or decreasing the reservoir pressure below hydrate equilibrium. Recent production tests in Alaska (Schoderbek et al., 2012), northern Canada (Ashford et al., 2012), and offshore Japan (Yamamoto et al., 2014) have shown that natural gas can be produced from gas hydrates with existing conventional oil and gas production technology. Exploration efforts designed to confirm the occurrence of recoverable gas hydrate in marine settings have produced positive results in Japan (Tsuji et al., 2009) and the Gulf of Mexico (Boswell et al., 2012a), and the pace of gas hydrate energy-assessment projects continues to accelerate. Beyond a future energy resource, gas hydrate may, in some cases, represent a significant drilling and production hazard (reviewed by Collett et al., 2009; Boswell et al., 2012b). Russian, Canadian, and American researchers have described problems associated with the occurrence of gas hydrate in the Arctic, including well control difficulties and casing failures (Collett et al., 2009). Other studies have also attempted to determine if naturally destabilized gas hydrate may contribute to the buildup of atmospheric methane, a significant greenhouse gas (Ruppel, 2011).

With growing interest in natural gas hydrates as a source of energy, a geologic hazard, and a potential agent of climate change, it is becoming increasingly important to be able to understand the physical nature and the geologic controls on the formation and stability of gas hydrate in nature. Knowledge of naturally occurring gas hydrates is growing and it can be concluded that (as reviewed by Collett et al., 2009): (1) a large volume of natural gas is present in gas hydrates; (2) production of natural gas from gas hydrates is technically feasible with existing technology; (3) gas hydrates hold the potential for natural hazards associated with seafloor stability and release of methane to the oceans and atmosphere; and (4) gas hydrates disturbed during drilling and conventional petroleum production pose a potential safety problem.

The Indian National Gas Hydrate Program Expedition 01 (NGHP01 ) is designed to study the gas hydrate occurrences off the Indian Peninsula and along the Andaman convergent margin, with special emphasis on understanding the geologic and geochemical controls on the occurrence of gas hydrate in these two diverse settings (Rastogi et al., 1999; Ramana et al., 2006). An additional aspect of this expedition was to further develop and calibrate geophysical tools, including multi-channel seismic (MCS) data, to the presence and concentration of gas hydrates. Analysis of available industryacquired downhole $\log$ and seismic data from the Krishna-Godavari offshore region (acquired before NGHP-01) indicate that the distribution of gas hydrate can be highly variable (reviewed by Collett et al., 2009). Therefore, the development of a more complete understanding of the regional and local gas hydrate systems was another goal of this expedition.

This report is intended to provide an overview of the occurrence of gas hydrates in the offshore of India and summarize the operational and technical results of the NGHP-01 expedition. This report begins with a review of earlier gas hydrate research efforts in India that led to formation of the NGHP. The main body of the report reviews planning and operational phases of the NGHP-01 expedition, which is followed by detailed descriptions of the NGHP-01 drilling results and the evidence for the occurrence of gas hydrates in the Krishna-Godavari, the Mahanadi, and the Kerala-Konkan Basins, and the Andaman Sea.

\section{Gas hydrates in the offshore of India}

\subsection{Earlier investigations}

The first indication of the presence of gas hydrates in the offshore India dates to 1984, when the Oil and Natural Gas Corporation Limited (ONGC) (Chopra, 1985) identified bottom-simulating reflectors (BSRs), which are often attributed to the presence of gas hydrate, on seismic lines in the Andaman Island offshore area. In 1997, the National Institute of Oceanography (NIO) generated the first gas hydrate resource map of India, which depicts the gas hydrate stability zone (GHSZ) in the Indian offshore. A similar exercise was carried out by Rao (2001) (Fig. 1). By 1997, a large number of multi-channel seismic reflection surveys had been conducted by the oil and gas industry for exploration of hydrocarbons in the Indian deepwater basins beyond the $400 \mathrm{~m}$ isobath. These data were examined for geophysical evidence of gas hydrates and a map, based mostly on the presence of BSRs, was produced that showed areas with evidence for the presence of gas hydrates, covering about 80,000 square kilometers of the outer continental shelf of India (Chandra et al., 1998). During the same period, ONGC in collaboration with the University of Victoria, used reprocessed seismic lines to document prominent BSRs in the Krishna-Godavari Basin. The Directorate General of Hydrocarbons (DGH) also conducted gas hydrate-related studies using 


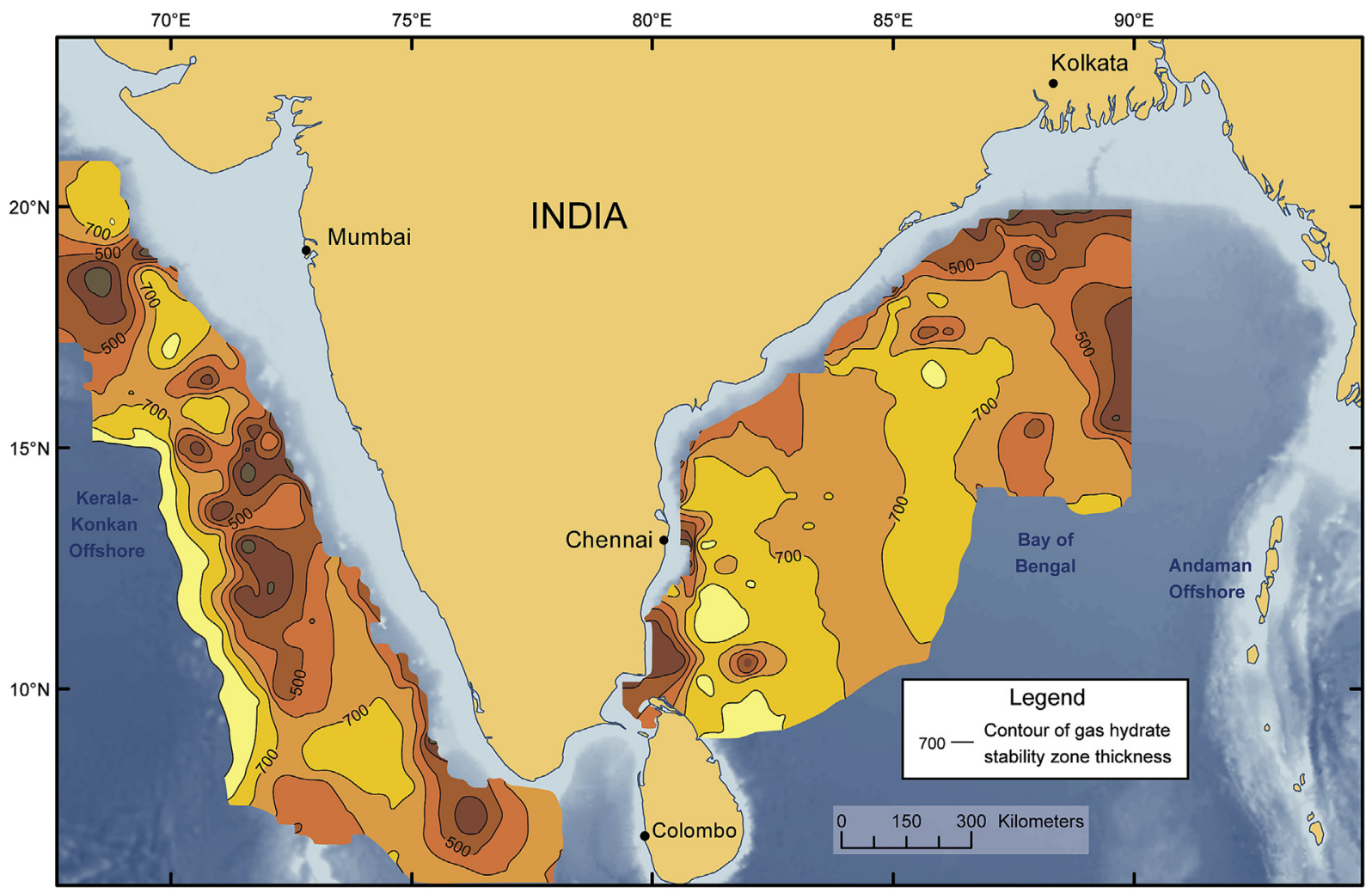

Figure 1. Gas (methane) hydrate stability zone thickness map along the continental margin of India (modified from Rao, 2001). Contour interval = 100 m.

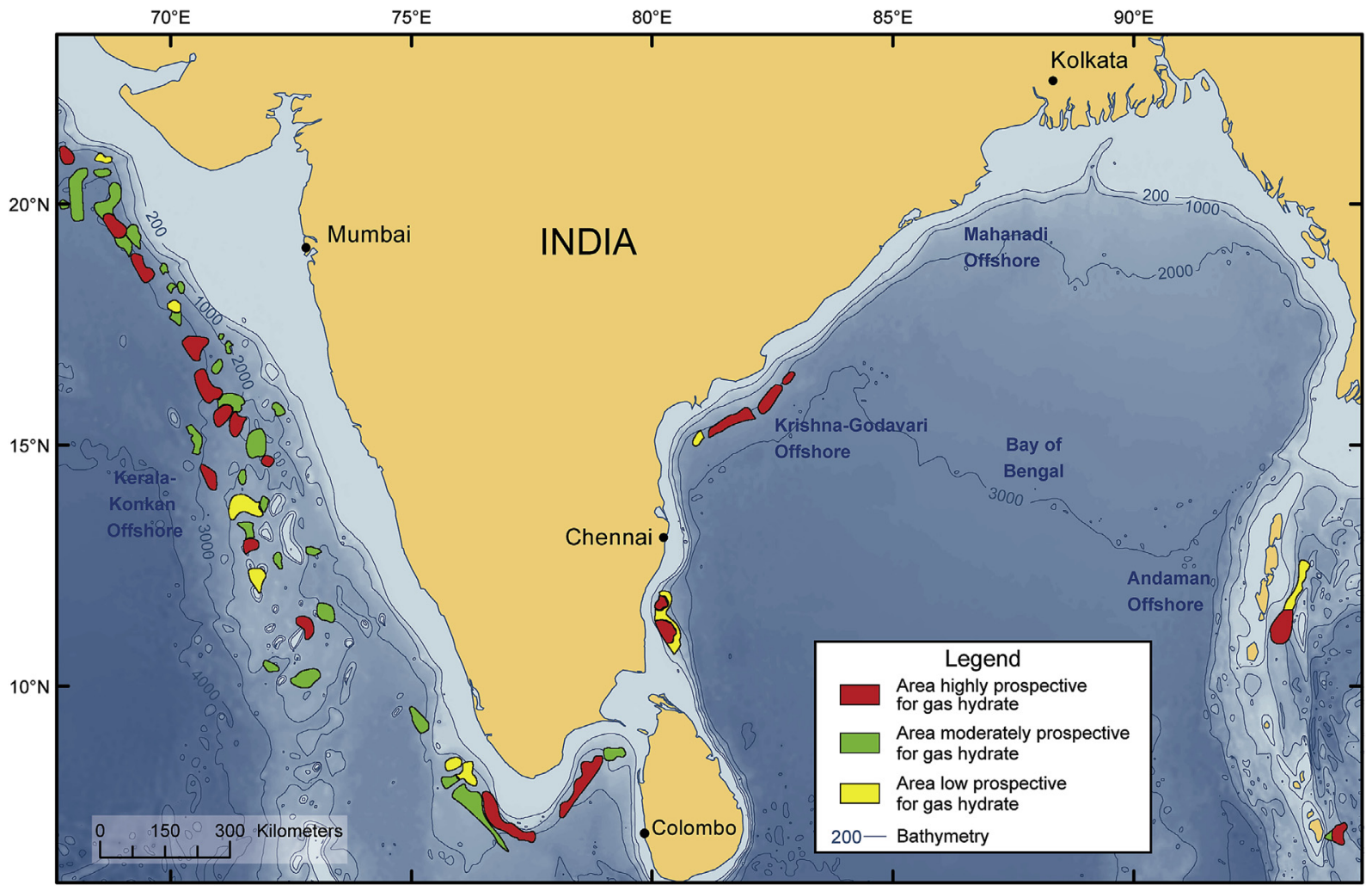

Figure 2. Map depicting prospective areas for gas hydrates along the Indian continental margins (modified from Chandra et al., 1998; Ramana et al., 2006). 
seismic data that had been acquired from the Andaman offshore area (Sethi et al., 2004). BSRs were observed cross-cutting several large anticlinal features and it was suggested that gas hydrate was acting as a "cap for underlying free-gas" accumulations. The Gas Authority of India Limited (GAIL), in partnership with NIO and the National Geophysical Research Institute(NGRI), conducted gas hydrate studies using $4300 \mathrm{~km}$ of multi-channel seismic data from the Kerala-Konkan Basin on the west coast of India, an area characterized by weak to strong BSRs. Finally, in 1998, Chandra et al. (1998) published a map that depicted the "prospective areas" for gas hydrates in the offshore of India (Fig. 2). A similar exercise and series of related studies were also conducted by NGRI (Rao, 1999, 2001; Rao et al., 1998, 2001a, 2001b, 2002).

\subsection{India National Gas Hydrate Program (NGHP)}

The Indian Geophysical Union (IGU) Conference held in 1995 at NGRI in Hyderabad, included a review session on the occurrence of gas hydrates in the offshore of India (Ramana et al., 2006). Subsequently, the Ministry of Petroleum \& Natural Gas (MOP\&NG) convened an expert committee to recommend the steps to be taken for exploration and development of gas hydrate resources in India. The National Gas Hydrate Program (NGHP) was established in 1997 from the recommendations of this committee. In the year 2000, the NGHP was reconstituted by the MOP\&NG under the overall coordination of DGH and monitored by a Steering Committee chaired by the Secretary of the MOP\&NG. The ultimate goal of the NGHP effort was to "achieve maximum self-sufficiency in hydrocarbons through tapping this unconventional source of energy." In addition to the NGHP Steering Committee, the NGHP includes a Technical Committee (with representatives from MOP\&NG, DGH, ONGC, GAIL, Oil India (OIL), and NIO and NGRI from the Council of Scientific and Industrial Research, National Institute of Ocean Technology (NIOT), and the Department of Defense) that is comprised of five Operational Subgroups: (1) Drilling, (2) Production, (3) Geosciences, (4) Environment, and (5) Transportation.

Based on the results of multi-disciplinary studies carried out in collaboration under the NGHP with DGH, ONGC, GAIL, and NIO, three offshore areas were identified for "further geoscience investigation": Krishna-Godavari Basin, Kerala-Konkan Basin, and the Andaman Island offshore area. Various geologic and geophysical surveys were conducted along the west and east coast of India in 2002-2003 (Ramana et al., 2006), which included the acquisition of bathymetry data, deep-tow digital side scan sonar images, chirp sonar, gravity and magnetic data, and marine gravity core-derived geochemical and microbiological data. These studies revealed a southwest to northeast trending positive topographic feature in the Krishna-Godavari Basin that rises from surrounding water depths of about $1400 \mathrm{~m}$ to as shallow as $900 \mathrm{~m}$. The study of gravity cores revealed evidence of methane gas enrichment along the crest of this feature, which further suggested the geology of the Krishna-Godavari Basin is highly conducive for the presence of gas hydrate. This theory was further strengthened by the discovery of hydrate-bearing layers in several oil and gas industry exploratory wells drilled in the basin (Collett et al., 2008a). ONGC also applied advanced acoustic inversion techniques to analyze a set of 12 seismic lines from the Krishna-Godavari Basin that allowed them to estimate that the area included in this study may contain as much as 350-900 billion cubic meters of gas in the form of gas hydrates (Varma, 2004). These studies eventually led to the selection of the Krishna-Godavari Basin as one of the areas included in the NGHP-01 drill site selection review. Multidisciplinary studies of the Kerala-Konkan Basin also revealed a noteworthy northwestsoutheast trending ridge that became a focus of the NGHP-01 pre-expedition site review process. The seismic evidence for the occurrence of gas hydrates in the Andaman Island offshore area led it to be selected for the NGHP-01 drill site selection review.

\subsection{India Nation Gas Hydrate Program Expedition 01 - planning and operations}

From an operational and scientific perspective, the NGHP-01 Expedition followed the general objectives for gas hydrate research drilling as proposed by the Ocean Drilling Program (ODP) Gas Hydrate Program Planning Group. These science objectives were further developed and modified from the NGHP gas hydrate research "road map" (Directorate General of Hydrocarbons 2014). The primary science objectives of this effort were to: (1) study the formation of natural gas hydrate in marine sediments; (2) determine the geologic controls on the formation and occurrence of gas hydrate in nature; (3) investigate gas-transport mechanisms and migration pathways from source to reservoir; (4) examine the effect of gas hydrate on the physical properties of the host sediments; (5) investigate the microbiology and geochemistry of gas hydrate formation and dissociation; and (6) calibrate geophysical and other predictive tools to the observed presence and concentration of gas hydrate. Even though the NGHP effort in India was founded to narrowly focus on assessing the energy resource potential of gas hydrates in the offshore of India, the objectives of the NGHP-01 expedition dealt with analysis of a broad set of science issues that serve as the foundation for the general study of gas hydrates in nature. These basic research objectives and scientific results make up the content of the contributions to this special issue of the Journal of Marine and Petroleum Geology.

NGHP-01 was planned and managed through a collaboration between the DGH under the MOP\&NG, the U.S. Geological Survey (USGS), and the Consortium for Scientific Methane Hydrate Investigations, led by Overseas Drilling Limited (ODL) and FUGRO McClelland Marine Geosciences (FUGRO). The platform for drilling operations was the research $D / V$ JOIDES Resolution, operated by ODL. Most of the drilling/coring equipment was provided by the Integrated Ocean Drilling Program (IODP) through a loan agreement with the U.S. National Science Foundation. Wireline pressure coring systems and supporting laboratories were provided by IODP/ Texas A\&M University, FUGRO, USGS, the U.S. Department of Energy (USDOE), and Geotek Ltd. Operational and technical support for downhole logging was provided by the Lamont-Doherty Earth Observatory of Columbia University.

The pre-expedition site review and selection process first focused on the occurrence of seismically identified BSRs that were inferred to indicate the occurrence of gas hydrate. In addition, the sedimentary section above the BSR was examined for evidence of potential gas hydrate occurrences. On-going studies of the use of seismic data to identify high-concentration gas hydrate deposits, in the Arctic (see Lee et al., 2009; Inks et al., 2009) and also in the marine environment (McConnell and Zhang, 2005), suggested good potential for applying existing hydrocarbon exploration approaches to prospect for marine gas hydrates (see Collett et al., 2009). In general, consensus was emerging that high-amplitude seismic reflectors of appropriate polarity within the interpreted gas hydrate stability zone can reveal the occurrence of relatively thick, highly saturated gas hydrate reservoirs. Thus, in the NGHP-01 site review process, attention was given to identifying high-amplitude reflectors and seismic-facies evidence for potential channelized deposits using available 2-D seismic data. One of the primary gas hydrate reservoir targets was a series of prominent cut-and-fill canyon-like features along the eastern margin of India that may contain coarser-grained channel-levee deposits. The preexpedition site review process also included a pre-drilling geohazard assessment of each planned drill site. A total of 19 potential 
drill sites were assessed and a geohazard report was provided by FUGRO Geoservices, Inc. (Wegner and Campbell, this issue).

The pre-expedition site review process yielded a total of 10 proposed drill sites, which exhibited variable geologic conditions and seismic responses indicative of gas hydrate-bearing sediments: one site in the Kerala-Konkan area on the west coast of India, eight sites on the east coast of India including six sites in the Krishna-Godavari Basin, and two sites in the Mahanadi Basin, and one drill site proposed for the convergent margin setting of the Andaman Islands. For the sites on the east coast of India, it was proposed to drill three holes at each, with the first hole dedicated to loggingwhile-drilling (LWD) operations in order to identify intervals to be targeted for later pressure coring. The second hole at each site would be dedicated for continuous conventional wireline coring. The third hole was to be used for special downhole tool measurements, with pressure coring at intervals of high interest as indicated in the LWD data and wireline logging.

For organizational purposes this project was divided into three phases:

Phase I - Expedition Planning and Mobilization: This project started with the mobilization of the scientific D/V JOIDES Resolution from Galveston, Texas to Mumbai, India, and with the staffing of the science team and the development of a project prospectus.

Phase II - Field Project Management, Operations, Core Analysis, and Well Log Data Characterization: The operational phase of NGHP-01 began with the arrival of the scientific crew in Mumbai, India on 28 April 2006 and ended 113.5 days later with the departure of ship from its final berth in Chennai on 19 August 2006. The expedition consisted of five separate "legs" as follows:

Leg 1 ( 5 May - 16 May): Sailed southwest from Mumbai to a location in the Kerala-Konkan Basin, Arabian Sea; conducted drilling, logging, and coring operations; then sailed around the southern tip of India to port in Chennai.

Leg 2 (19 May - 6 June): Conducted personnel and equipment transfers in Chennai, then sailed to ten sites in the Krishna-Godavari and Mahanadi Basins; conducted LWD operations; returned to Chennai for offloading of the LWD tools and engineers.

Leg 3A (9 June - 25 June): Informed with the LWD results, sailed to four selected sites within Krishna-Godavari Basin for drilling, coring, and wireline logging operations, before returning to Chennai for personnel and equipment transfers. Leg 3B ( 28 June - 17 July): Conducted additional drilling, coring, and logging operations at five sites within the Krishna-Godavari Basin.

Leg 4 (17 July - 17 August): After personnel transfers via helicopter, sailed east and cored and logged a site east of Little Andaman Island, then sailed northwest for two sites within the Mahanadi Basin, then sailed southwest to drill two additional sites within the Krishna-Godavari Basin, before sailing to Chennai. Drilling, coring, and logging operations were conducted at each site established during Leg 4. Phase III - Demobilization and Collaborative Post-Field Project Analysis of Geologic Data and Samples: The project included a wide range of collaborative post-field analysis of samples collected during the expedition and reporting of the geologic results of this effort. All of the primary data collected during the expedition are included in either the NGHP Expedition 01 Initial Reports (Collett et al., 2008a) or the NGHP Expedition 01 Downhole Log Data Report (Collett et al., 2008b), which were prepared by the USGS and published by the DGH on behalf the MOP\&NG. Phase III of this project also provided for the publication of the NGHP Expedition 01 Scientific Results Volume, which is represented by this Special Issue of the Journal of Marine and Petroleum Geology.

\subsection{India National Gas Hydrate Program Expedition 01 - drilling results and occurrence of gas hydrate}

\subsubsection{General remarks}

NGHP-01 began at $0552 \mathrm{~h}$ on 28 April 2006 with the arrival of the $D / V$ JOIDES Resolution in Mumbai, India, ending 113.5 days later in Chennai, India at 1912 h on 19 August (Figs. 3 and 4; Tables 1-4). Ultimately, 21 sites were occupied and five sites were revisited at least once. Thirty-nine holes were drilled. Of these, 12 holes were LWD drilled, 24 holes were cored, and 3 holes were drilled as dedicated wireline logging holes. Thirteen holes were wireline logged and temperature gradients were established in 11 holes.

This section of the expedition summary includes descriptions of the methods used by the Shipboard Science Party in the operation, downhole logging, sample collection and handling, shipboard laboratory analyses, and data analysis during the course of NGHP-01. This expedition, while not an Integrated Ocean Drilling Program (IODP) activity, was specifically designed to conform to IODP operational protocols.

\subsubsection{NGHP-01 drilling and coring operations}

Holes were produced through the use of a variety of standard coring systems and specialized pressure coring systems, as well as through standard drilling in the case of LWD and dedicated wireline logging holes. Conventional coring systems used included the IODP's Advanced Piston Corer (APC) and the Extended Core Barrel (XCB). Both systems collect cores approximately $9.6 \mathrm{~m}$ in length within $6.7 \mathrm{~cm}$ diameter clear plastic core liners. The typical approach to coring was to utilize the APC corer until first refusal (an incomplete core indicating failure to fully penetrate the sediment), then switch to XCB coring to the full well depth. Pressure coring systems were employed at regular intervals or to target specific zones of interest observed in previously drilled LWD holes (Leg 2) or on seismic data (Table 3). Pressure coring systems used include the Pressure Coring System (PCS), the Hyacinth Rotary Corer (HRC), and the Fugro Pressure Corer (FPC). Each of these systems collects cores approximately $1.0 \mathrm{~m}$ in length, with the following diameters: FPC $-5.7 \mathrm{~cm}$; HRC $-5.1 \mathrm{~cm}$; PCS $-4.3 \mathrm{~cm}$. Dedicated logging holes were typically drilled with the insertion of a center bit to the XCB system. In addition, in situ temperature measurements were made with a variety of devices (Table 4), including the Davis-Villinger Temperature Pressure probe (DVTP) when XCB coring and the APCT or APCT -3 when APC coring. During NGHP-01, drilling depths were measured initially in terms of meters below rig floor (mbrf). From these values, depths in meters below sea floor (mbsf) were calculated by subtracting the water depth.

Cores acquired during NGHP-01 were generally handled according to ODP/IODP procedures (Paull et al., 1996; Tréhu et al., 2004; Riedel et al., 2006). Modifications to these procedures, similar to those made in ODP Leg 204 and IODP Expedition 311 (also gas hydrate focused expeditions), were designed to enable quick identification of gas hydrate-bearing intervals, maintain aseptic conditions for microbiological sampling, and identify and safely manage hydrogen sulfide gas. For a complete description of the procedures used to handle and process all of the sediment cores acquired during NGHP-01 see the "Methods" section of the NGHP Expedition 01 Initial Reports (Collett et al., 2008a).

For most of the sites cored during NGHP-01, inspection of the cored sediment began immediately upon recovery with infrared 

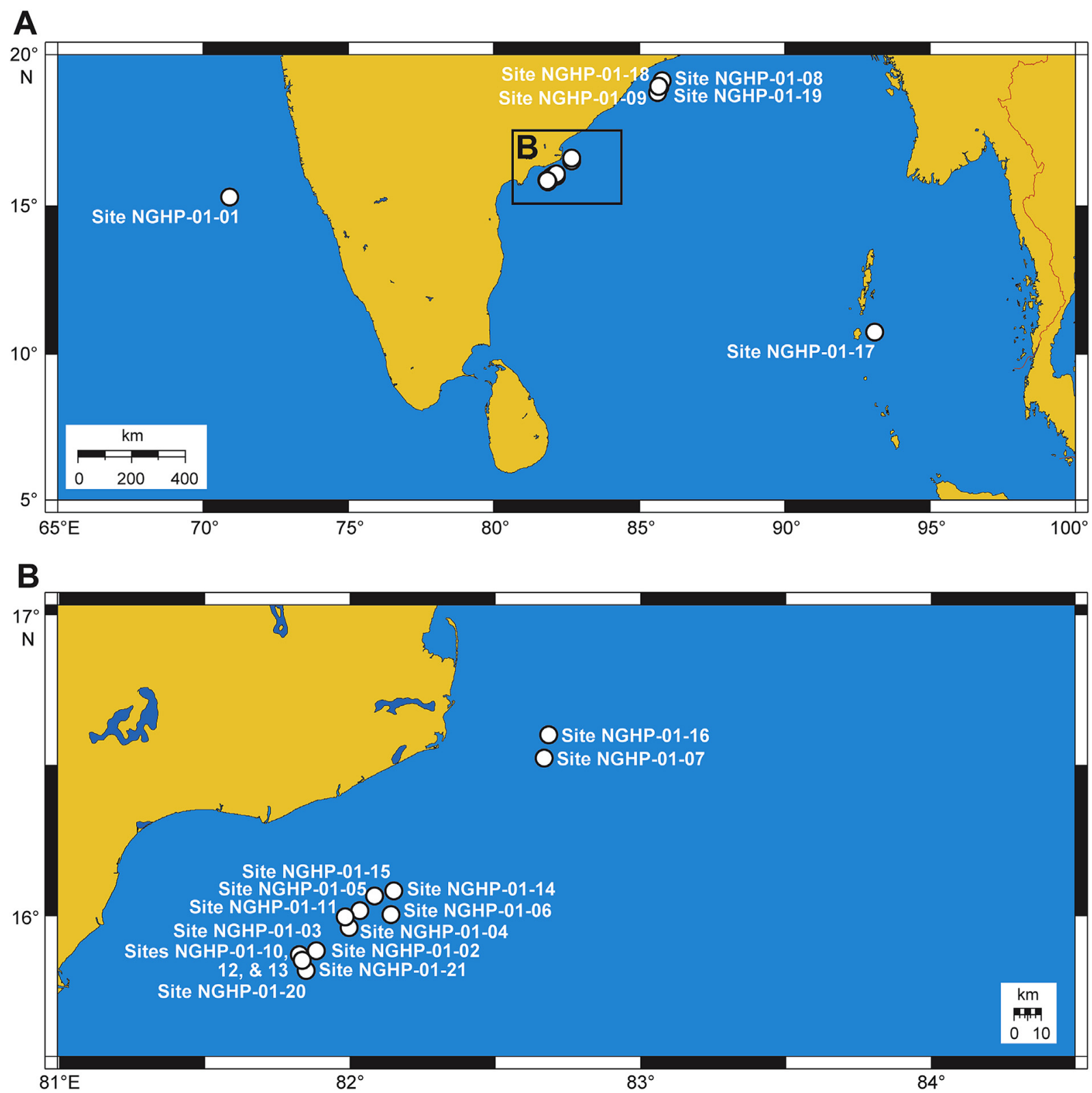

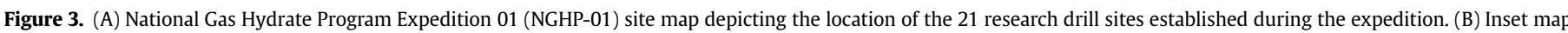
of the drill sites in the Krishna-Godavari Basin (modified from Collett et al., 2008a).

(IR) imaging of the core while still in the liner. These scans were used primarily to identify anomalously cold sections of liner that are indicative of recent or ongoing gas hydrate dissociation within the enclosed sediments (Tréhu et al., 2004). Many observed thermal anomalies were immediately cut from the core and either: (1) sent to the geochemistry lab for visual inspection and extraction of interstitial waters for geochemical analyses; (2) sampled and stored for later shore-based microbiological studies; or (3) bagged and preserved in liquid nitrogen-filled dewars or methane-charged pressure vessels. Visible gas hydrate samples obtained from the core catcher or otherwise extruded from the core liner were stored in liquid nitrogen. In most cases, a background sample (nonanomalous) was taken from each core for immediate geochemical analyses. Gas samples were routinely collected from void spaces within the cores and analyzed onboard in the geochemistry laboratory. The remainder of the core was then measured for sectioning and labeled. A suite of pre-planned samples were then cut, capped, labeled, and stored as appropriate for each sample type. The remaining core sections were transferred to the ship core processing laboratory and allowed to equilibrate to room temperature before further processing.

In the core processing laboratory, the remaining whole-round core sections were first tested for thermal conductivity, then passed through the multisensor core logger (MSCL) to obtain measurements of compressional-wave velocity, noncontact resistivity, gamma-ray attenuation bulk density, and magnetic susceptibility (Collett et al., 2008a). The cores were then split, from bottom to top, creating separate archive and working halves. The working half was then sampled for further physical properties testing (for example moisture and density, shear strength, splitcore acoustic velocity, and contact resistivity) (Collett et al., 2008a). The working half of the core was then transferred to the sampling table where it was further subsampled. Subsampling included the regular collection of background material for later sedimentological, X-ray diffraction, carbon dating, and paleomagnetism studies, as well as targeted sampling of notable features 


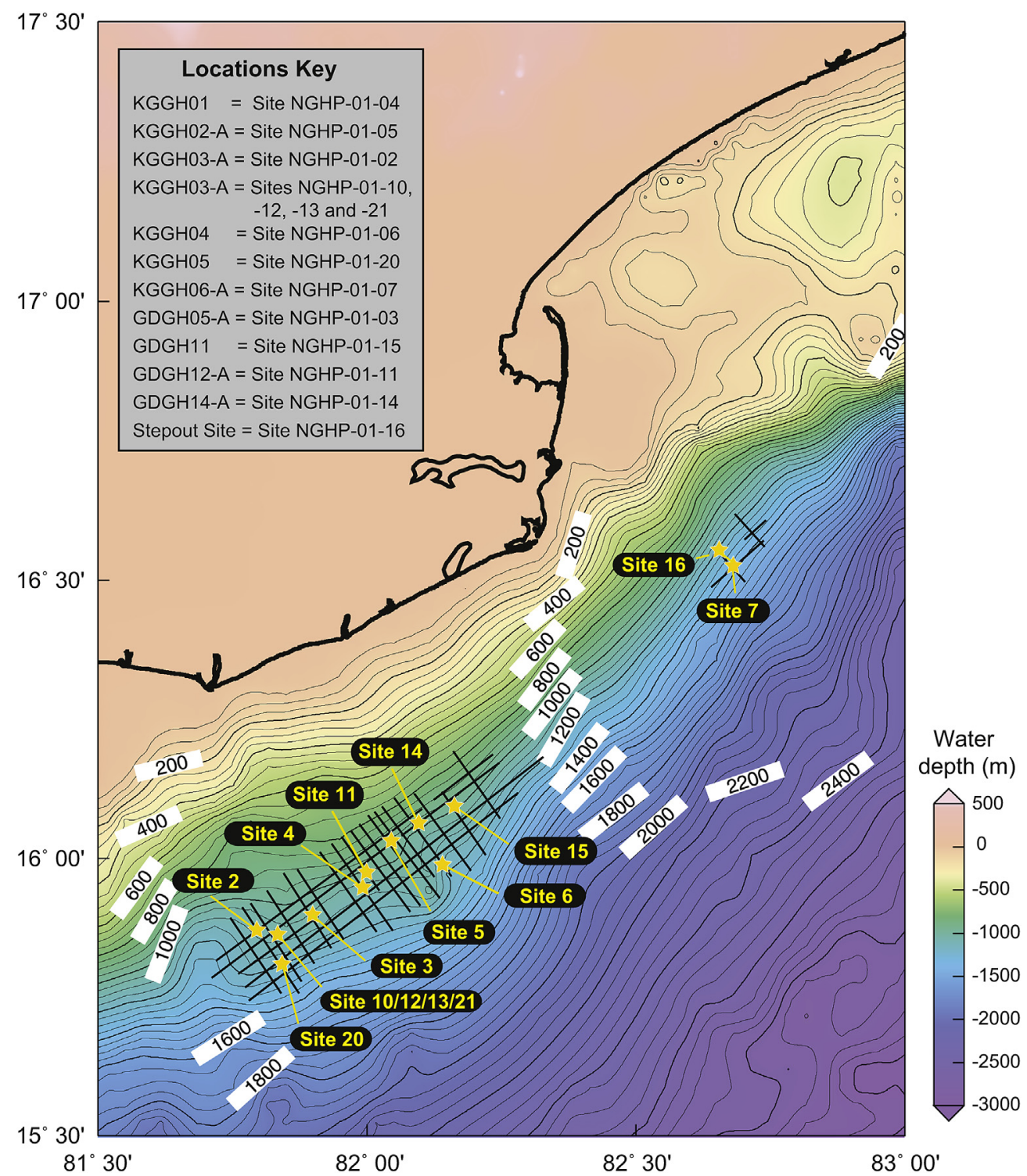

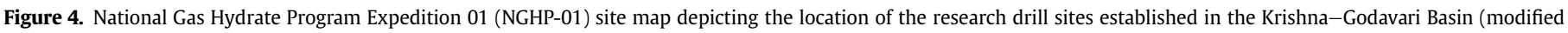
from Collett et al., 2008a).

such as carbonate nodules, volcanic ash layers, macrofossils, and other features.

The archive half-core sections were scanned on the digital imaging system (DIS). Visual core descriptions of the archive halves were prepared and augmented by microscopic analyses of smear slides. Digital close-up photographs were taken of particular features for illustrations, as requested by scientists. Both halves of the core were then placed in labeled plastic tubes and transferred to cold storage for the remainder of the expedition.

For pressure core processing and analyses, two 20-ft refrigerated containers were mounted on the vessel during NGHP-01. The lab stack van (also referred to as the PCS van) contained the PCS degassing manifold, and a horizontal multisensor core logger (MSCL). The core technician shop container (also referred to as the HYACINTH van) housed the HYACINTH transfer system and degassing manifold, as well as a pressure multisensor core logger (MSCL-P).

Pressure cores retrieved at or near in situ pressures were used to determine gas hydrate distribution and quantity using nondestructive measurement of the physical properties of the cores at in situ pressures, and destructive methane collection and mass balance measurements (Tables 2 and 3). The techniques used to collect and analyze the recovered pressure cores on NGHP-01 were similar to the procedures employed on IODP Expedition 311 (Riedel et al., 2006; Collett et al., 2008a), which have been further refined on subsequent marine gas hydrate expeditions as reviewed by Schultheiss et al. (2009). After a pressure core was retrieved, initial non-destructive measurements, including gamma density and compressional-wave velocity, as well as X-ray images were used to characterize the nature and distribution of gas hydrate within the sediment. Some cores were then depressurized on the ship to determine total methane composition and concentration in sediments, or stored at in situ pressure for further analyses on shore. All depressurized cores had non-destructive measurements taken while undergoing depressurization and again following depressurization to document gas evolution, gas hydrate dissociation, and other changes in the core. The released gas volume and nondestructive measurements were used to guide subsampling for interstitial water, physical properties, and other related analyses.

At the conclusion of the expedition, selected cores and samples were offloaded in Chennai in refrigerated containers and transported to the National Gas Hydrate Program Repository in Mumbai, 
Table 1

National Gas Hydrate Program Expedition 01 site summary data, including listing of pre-expedition prospectus site designation, leg number, site number, water depth as determined by drilling or coring, depth to the base of methane hydrate stability zone, and depth of the seismically identified bottom simulating reflector (BSR) (modified from Collett et al., 2008a). NA = not available, mbsf $=$ meters below seafloor.

\begin{tabular}{|c|c|c|c|c|c|}
\hline $\begin{array}{l}\text { Prospectus } \\
\text { designation }\end{array}$ & Leg & $\begin{array}{l}\text { Site } \\
\text { number }\end{array}$ & $\begin{array}{l}\text { Water } \\
\text { depth } \\
(\mathrm{m})\end{array}$ & $\begin{array}{l}\text { Depth to base of } \\
\text { methane hydrate } \\
\text { stability zone } \\
\text { (mbsf) }\end{array}$ & $\begin{array}{l}\text { BSR } \\
\text { depth } \\
\text { (mbsf) }\end{array}$ \\
\hline KKGH01 & 1 & NGHP-01-01 & 2663 & 360 & no BSR \\
\hline KGGH03-A & 2 & NGHP-01-02 & 1058 & NA & 170 \\
\hline GDGH05-A & 2 & NGHP-01-03 & 1076 & 203 & 209 \\
\hline KGGH01 & 2 & NGHP-01-04 & 1081 & NA & 182 \\
\hline KGGH02-A & 2 & NGHP-01-05 & 945 & 130 & $\sim 125$ \\
\hline KGGH04 & 2 & NGHP-01-06 & 1160 & NA & 210 \\
\hline KGGH06-A & 2 & NGHP-01-07 & 1285 & 198 & 188 \\
\hline MNGH01-1-A & 2 & NGHP-01-08 & 1689 & NA & 257 \\
\hline MNGH-01-2 & 2 & NGHP-01-09 & 1935 & NA & $\sim 290$ \\
\hline KGGH03-A (GD-3-1) & 2 & NGHP-01-10 & 1038 & 160 & $\sim 160$ \\
\hline GDGH12-A & 2 & NGHP-01-11 & 1007 & NA & 150 \\
\hline KGGH03-A (1st New Site) & $3 \mathrm{~A}$ & NGHP-01-12 & 1038 & NA & $\sim 160$ \\
\hline KGGH03-A (2nd New Site) & $3 \mathrm{~A}$ & NGHP-01-13 & 1038 & NA & $\sim 160$ \\
\hline GDGH14-A & $3 \mathrm{~B}$ & NGHP-01-14 & 895 & 150 & 109 \\
\hline GDGH11 & $3 \mathrm{~B}$ & NGHP-01-15 & 926 & 126 & 126 \\
\hline Stepout Site & $3 \mathrm{~B}$ & NGHP-01-16 & 1253 & 178 & 170 \\
\hline ANGH01 & 4 & NGHP-01-17 & 1344 & 620 & $\sim 608$ \\
\hline MNGH-REL 5 & 4 & NGHP-01-18 & 1374 & 210 & $\sim 210$ \\
\hline MNGH-Gap & 4 & NGHP-01-19 & 1422 & 220 & 205 \\
\hline KGGH05 & 4 & NGHP-01-20 & 1146 & NA & $\sim 220$ \\
\hline KGGH03-A (New FR1) & 4 & NGHP-01-21 & 1049 & NA & $\sim 160$ \\
\hline
\end{tabular}

India, or shipped to various researchers in India and elsewhere. The remainder of the cores and samples stayed on the vessel and were taken to Singapore, where they were shipped to a group of approved post-expedition laboratories in the United States, Canada, and Germany for further analysis. Five of the pressure cores were stored for further post-expedition analysis in Singapore (Collett et al., 2008a; Holland and Schultheiss, in this issue).

\subsubsection{NGHP-01 coring performance and assessment}

During NGHP-01, there were 494 conventional (non-pressurized) wireline cores cut and $2847.01 \mathrm{~m}$ of core recovered, which reflected 78.7 percent of the interval cored (Table 2). The APC coring system recovered 125 cores with $1116.74 \mathrm{~m}$ of core recovery, which is 101.6 percent of the formation penetrated (excess recovered core is because of core expansion during recovery). The XCB coring system recovered 272 cores with $1661.79 \mathrm{~m}$ of core recovery which is $68.6 \%$ of the formation penetrated. A total of 76 in situ temperature measurements were also attempted using three available wireline temperature tools (Table 4). The APCT was deployed 7 times, the APCT-3 a total of 27 times, and the DVTP 42 times. Using this data, formation temperature gradients were established for each site where wireline coring was conducted.

During NGHP-01, the three available pressure core systems were deployed 97 times and exhibited an overall success rate of $41 \%$ (Table 3). A "successful" pressure core was defined as a pressure core with $>30 \%$ recovery and retained $>50$ bar ( 725 psi) of pressure. "Successful" pressure cores provided a good assessment of the concentration of gas hydrate in the core, as the core was unlikely to have lost any significant constituents. Cores that provided the highest quality data about both gas hydrate morphology and distribution as well as the concentration of gas hydrate within the sediment were termed "excellent" pressure cores, which were defined as $>60 \%$ sediment recovery, $>70 \%$ in situ pressure, and $>50$ bar pressure. Out of 42 deployments, the PCS returned 12 successful cores (29\%), 8 of which were considered excellent cores (19\%). Overall, the PCS was very reliable in recovering full cores in most formations cored on this expedition. Out of 31 deployments, the FPC returned 12 successful cores (39\%), all of which were considered excellent cores. Out of 24 deployments, the HRC returned 16 successful cores (67\%), 10 of which were considered excellent cores (42\%). The pressure core success rate for all of the systems used during NGHP-01 steadily improved throughout the course of the expedition as experience with the tools continued to grow.

\subsubsection{NGHP-01 LWD and wireline operations}

Throughout NGHP-01, a variety of data were obtained through LWD, wireline logging in cored holes, and wireline logging in dedicated logging holes (Table 2). To facilitate the project schedule and budget, all LWD was conducted during one leg of the program (Leg 2). These pre-coring logging operations were critical to the development of the detailed, site-specific coring, core sampling, and pressure core deployment plans. The reported LWD and wireline well log depths were measured from the seafloor as identified through the drill pipe on the recorded gamma-ray logs in each hole.

Twelve LWD holes were drilled at ten different sites during Leg 2 (Table 2). Penetration depths varied from 50.3 mbsf to 350.0 mbsf, and $2995.8 \mathrm{~m}$ of formation was logged during the LWD leg. Schlumberger Drilling and Measurements provided the following tools under contract with the Lamont-Doherty Earth Observatory Borehole Research Group (LDEO-BRG): GeoVISION (RAB), EcoScope, SonicVISION, TeleSCOPE, and ProVISION (NMR). Figure 5 shows the configuration of the LWD bottom hole assembly (BHA).

During NGHP-01, three wireline logging tool strings were deployed (Fig. 6): (1) the triple combination (or Triple-Combo) string (resistivity, density, and porosity measurements), which consists of the Hostile Environment Gamma Ray Sonde (HNGS), the Phasor Dual Induction (DIT) tool, the Hostile Environment LithoDensity Tool (HLDT), and the Accelerator Porosity Sonde (APS); (2) the FMS-sonic tool string (or FMS-Sonic), which consists of the FMS, General Purpose Inclinometer Tool (GPIT), and Scintillation Gamma Ray (SGT) Tool, and the Dipole Sonic Imager (DSI); and (3) the Vertical Seismic Imager (or VSI). The standard "triple combo" $\log$ and FMS-sonic tool strings were deployed at most of the sites. Both compressional- and shear-wave velocity measurements were made using the DSI tool. Depth-to-seismic ties were accomplished by means of synthetic seismograms constructed from the wireline density and acoustic logs. High-resolution formation microscanner (FMS) electrical resistivity images complemented the LWD resistivity-at-bit (RAB) images and aided in the identification of thin beds, veins, and fractures in hydrate-bearing sediments. The wireline VSI tool was used to collect zero-offset vertical seismic profiles (VSPs) at most of the sites. Consistent with IODP protocols, all VSP operations were conducted during daylight hours and assisted by mammal watchers posted at both the bow and stern of the ship. No mammals were observed during any of the VSP operations.

\subsubsection{NGHP-01 operational summary}

For organizational purposes, NGHP-01 was divided into five operational legs (Table 2). The first leg consisted of a single hole cored and wireline logged in the Kerala-Konkan Basin off the western coast of India. The second leg was a transect comprised solely of LWD holes. During Leg 2,12 holes at 10 sites were drilled in the Krishna-Godavari and Mahanadi Basins off the east coast of India. Leg $3 \mathrm{~A}$ consisted of six cored holes and two dedicated wireline logging holes at four sites located in the Krishna-Godavari Basin. Two sites were re-occupied sites that were previously LWD drilled on Leg 2 and two sites were new. Leg 3B consisted of seven cored holes plus one hole that was abandoned due to hole 
Table 2

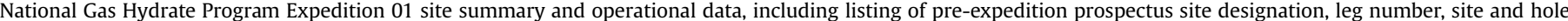

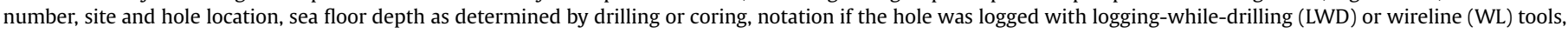

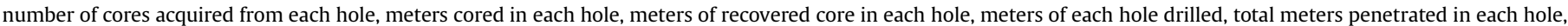
total depth of each hole, and the cumulative time working on each hole (modified from Collett et al., 2008a). mbrf = meters below rig floor.

\begin{tabular}{|c|c|c|c|c|c|c|c|c|c|c|c|c|c|c|c|c|c|}
\hline $\begin{array}{l}\text { Prospectus } \\
\text { designation }\end{array}$ & Leg & $\begin{array}{l}\text { Site/hole } \\
\text { no. }\end{array}$ & $\begin{array}{l}\text { Lati } \\
\text { (deg }\end{array}$ & $\begin{array}{l}\text { tude } \\
\text { / } / \mathrm{min})\end{array}$ & & $\begin{array}{l}\text { Longi } \\
\text { (deg/ }\end{array}$ & $\begin{array}{l}\text { itude } \\
\text { min) }\end{array}$ & & $\begin{array}{l}\text { Sea floor } \\
\text { depth } \\
\text { (mbrf) }\end{array}$ & $\begin{array}{l}\text { LWD/WL } \\
\text { logged }\end{array}$ & $\begin{array}{l}\text { No. of } \\
\text { cores }\end{array}$ & $\begin{array}{l}\text { Meters } \\
\text { cored }\end{array}$ & $\begin{array}{l}\text { Meters } \\
\text { recovered }\end{array}$ & $\begin{array}{l}\text { Meters } \\
\text { drilled }\end{array}$ & $\begin{array}{l}\text { Total } \\
\text { meters } \\
\text { penetration }\end{array}$ & $\begin{array}{l}\text { Total } \\
\text { depth } \\
\text { (mbrf) }\end{array}$ & $\begin{array}{l}\text { Time } \\
\text { on hole } \\
\text { (hours) }\end{array}$ \\
\hline \multirow[t]{2}{*}{ KKGH-01 } & 1 & NGHP-01-01A & $15^{\circ}$ & $18.366^{\prime}$ & $\mathrm{N}$ & $070^{\circ}$ & $54.192^{\prime}$ & $\mathrm{E}$ & 2674.2 & WL & 37 & 285.0 & 279.26 & 5.0 & 290.0 & 2964.2 & 93.00 \\
\hline & & & & & & & & & NGHP-01-01 TOTALS & & 37 & 285.0 & 279.26 & 5.0 & 290.0 & $\mathrm{~N} / \mathrm{A}$ & 93.00 \\
\hline \multirow[t]{3}{*}{ KGGH03-A } & 2 & NGHP-01-02A & $15^{\circ}$ & $52.1190^{\prime}$ & $\mathrm{N}$ & $081^{\circ}$ & $49.3583^{\prime}$ & $\mathrm{E}$ & 1069.0 & LWD & 0 & 0.0 & 0.00 & 50.3 & 50.3 & 1119.3 & 19.25 \\
\hline & 2 & NGHP-01-02B & $15^{\circ}$ & $52.1217^{\prime}$ & $\mathrm{N}$ & $081^{\circ}$ & $49.3583^{\prime}$ & $\mathrm{E}$ & 1069.0 & LWD & 0 & 0.0 & 0.00 & 250.0 & 250.0 & 1319.0 & 24.25 \\
\hline & & & & & & & & & NGHP-01-02 TOTALS & & 0 & 0.0 & 0.00 & 300.3 & 300.3 & $\mathrm{~N} / \mathrm{A}$ & 43.50 \\
\hline \multirow[t]{4}{*}{ GDGH05-A } & 2 & NGHP-01-03A & $15^{\circ}$ & $53.8919^{\prime}$ & $\mathrm{N}$ & $081^{\circ}$ & $53.9678^{\prime}$ & $\mathrm{E}$ & 1092.0 & LWD & 0 & 0.0 & 0.00 & 300.0 & 300.0 & 1392.0 & 30.00 \\
\hline & 3B & NGHP-01-03B & $15^{\circ}$ & $53.8881^{\prime}$ & $\mathrm{N}$ & $081^{\circ}$ & $53.9638^{\prime}$ & $\mathrm{E}$ & 1085.1 & & 39 & 300.0 & 255.33 & 0.0 & 300.0 & 1385.1 & 60.00 \\
\hline & 3B & NGHP-01-03C & $15^{\circ}$ & $53.8957^{\prime}$ & $\mathrm{N}$ & $081^{\circ}$ & $53.9718^{\prime}$ & $\mathrm{E}$ & 1086.0 & WL & 10 & 35.5 & 34.93 & 264.5 & 300.0 & 1386.0 & 70.25 \\
\hline & & & & & & & & & NGHP-01-03 TOTALS & & 49 & 335.5 & 290.26 & 564.5 & 900.0 & $\mathrm{~N} / \mathrm{A}$ & 160.25 \\
\hline \multirow[t]{2}{*}{ KGGH01 } & 2 & NGHP-01-04A & $15^{\circ}$ & $57.3794^{\prime}$ & $\mathrm{N}$ & $081^{\circ}$ & $59.4650^{\prime}$ & $\mathrm{E}$ & 1092.0 & LWD & 0 & 0.0 & 0.00 & 300.0 & 300.0 & 1392.0 & 27.50 \\
\hline & & & & & & & & & NGHP-01-04 TOTALS & & 0 & 0.0 & 0.00 & 300.0 & 300.0 & $\mathrm{~N} / \mathrm{A}$ & 27.50 \\
\hline \multirow[t]{6}{*}{ KGGH02-A } & 2 & NGHP-01-05A & $16^{\circ}$ & $01.7221^{\prime}$ & $\mathrm{N}$ & $082^{\circ}$ & $02.6773^{\prime}$ & $\mathrm{E}$ & 963.0 & LWD & 0 & 0.0 & 0.00 & 200.0 & 200.0 & 1163.0 & 25.50 \\
\hline & 2 & NGHP-01-05B & $16^{\circ}$ & $01.7264^{\prime}$ & $\mathrm{N}$ & $082^{\circ}$ & $02.6773^{\prime}$ & $\mathrm{E}$ & 952.0 & LWD & 0 & 0.0 & 0.00 & 200.0 & 200.0 & 1152.0 & 18.75 \\
\hline & $3 \mathrm{~A}$ & NGHP-01-05C & $16^{\circ}$ & $01.7172^{\prime}$ & $\mathrm{N}$ & $082^{\circ}$ & $02.6733^{\prime}$ & $\mathrm{E}$ & 957.2 & & 24 & 200.0 & 142.83 & 0.00 & 200.0 & 1157.2 & 38.50 \\
\hline & $3 \mathrm{~A}$ & NGHP-01-05D & $16^{\circ}$ & $01.7248^{\prime}$ & $\mathrm{N}$ & $082^{\circ}$ & $02.6813^{\prime}$ & $\mathrm{E}$ & 955.1 & & 12 & 43.9 & 39.75 & 157.1 & 201.0 & 1156.1 & 45.50 \\
\hline & $3 \mathrm{~A}$ & NGHP-01-05E & $16^{\circ}$ & $01.7248^{\prime}$ & $\mathrm{N}$ & $082^{\circ}$ & $02.6733^{\prime}$ & $\mathrm{E}$ & 955.1 & WL & 0 & 0.0 & 0.00 & 200.0 & 200.0 & 1155.1 & 41.50 \\
\hline & & & & & & & & & NGHP-01-05 TOTALS & & 36 & 243.9 & 182.58 & 757.1 & 1001.0 & $\mathrm{~N} / \mathrm{A}$ & 169.75 \\
\hline \multirow[t]{2}{*}{ KGGH04 } & 2 & NGHP-01-06A & $16^{\circ}$ & $00.0928^{\prime}$ & $\mathrm{N}$ & $082^{\circ}$ & $08.2767^{\prime}$ & $\mathrm{E}$ & 1171.0 & LWD & 0 & 0.0 & 0.00 & 350.0 & 350.0 & 1521.0 & 34.25 \\
\hline & & & & & & & & & NGHP-01-06 TOTALS & & 0 & 0.0 & 0.00 & 350.0 & 350.0 & $\mathrm{~N} / \mathrm{A}$ & 34.25 \\
\hline \multirow[t]{5}{*}{ KGGH06-A } & 2 & NGHP-01-07A & $16^{\circ}$ & $31.2785^{\prime}$ & $\mathrm{N}$ & $082^{\circ}$ & $40.8572^{\prime}$ & $\mathrm{E}$ & 1297.0 & LWD & 0 & 0.0 & 0.00 & 260.0 & 260.0 & 1557.0 & 25.75 \\
\hline & 3B & NGHP-01-07B & $16^{\circ}$ & $31.2747^{\prime}$ & $\mathrm{N}$ & $082^{\circ}$ & $40.8612^{\prime}$ & $\mathrm{E}$ & 1295.8 & & 31 & 211.6 & 180.28 & 0.0 & 211.6 & 1507.4 & 51.00 \\
\hline & 3B & NGHP-01-07C & $16^{\circ}$ & $31.2823^{\prime}$ & $\mathrm{N}$ & $082^{\circ}$ & $40.8532^{\prime}$ & $\mathrm{E}$ & 1295.8 & & 0 & 0.0 & 0.00 & 191.2 & 191.2 & 1487.0 & 15.25 \\
\hline & 3B & NGHP-01-07D & $16^{\circ}$ & $31.2823^{\prime}$ & $\mathrm{N}$ & $082^{\circ}$ & $40.8612^{\prime}$ & $\mathrm{E}$ & 1295.8 & WL & 4 & 28.8 & 32.65 & 231.2 & 260.0 & 1555.8 & 58.00 \\
\hline & & & & & & & & & NGHP-01-07 TOTALS & & 35 & 240.4 & 212.93 & 682.4 & 922.8 & $\mathrm{~N} / \mathrm{A}$ & 150.00 \\
\hline \multirow[t]{2}{*}{ MNGH01-1A } & 2 & NGHP-01-08A & $18^{\circ}$ & $57.4271^{\prime}$ & $\mathrm{N}$ & $085^{\circ}$ & $43.3793^{\prime}$ & $\mathrm{E}$ & 1700.0 & LWD & 0 & 0.0 & 0.00 & 350.0 & 350.0 & 2050.0 & 34.25 \\
\hline & & & & & & & & & NGHP-01-08 TOTALS & & 0 & 0.0 & 0.00 & 350.0 & 350.0 & $\mathrm{~N} / \mathrm{A}$ & 34.25 \\
\hline \multirow[t]{2}{*}{ MNGH01-2 } & 2 & NGHP-01-09A & $18^{\circ}$ & $46.0908^{\prime}$ & $\mathrm{N}$ & $085^{\circ}$ & $37.5022^{\prime}$ & $\mathrm{E}$ & 1946.0 & LWD & 0 & 0.0 & 0.00 & 330.0 & 330.0 & 2276.0 & 32.25 \\
\hline & & & & & & & & & NGHP-01-09 TOTALS & & 0 & 0.0 & 0.00 & 330.0 & 330.0 & $\mathrm{~N} / \mathrm{A}$ & 32.25 \\
\hline GD-3-1 & 2 & NGHP-01-10A & $15^{\circ}$ & $51.8609^{\prime}$ & $\mathrm{N}$ & $081^{\circ}$ & $50.0749^{\prime}$ & $\mathrm{E}$ & 1049.0 & LWD & 0 & 0.0 & 0.00 & 205.5 & 205.5 & 1254.5 & 26.75 \\
\hline & $3 \mathrm{~A}$ & NGHP-01-10B & $15^{\circ}$ & $51.8571^{\prime}$ & $\mathrm{N}$ & $081^{\circ}$ & $50.0789^{\prime}$ & $\mathrm{E}$ & 1049.4 & & 31 & 204.9 & 55.53 & 0.0 & 204.9 & 1254.3 & 61.25 \\
\hline & $3 \mathrm{~A}$ & NGHP-01-10C & $15^{\circ}$ & $51.8647^{\prime}$ & $\mathrm{N}$ & $081^{\circ}$ & $50.0709^{\prime}$ & $\mathrm{E}$ & 1050.4 & & 1 & 9.5 & 8.82 & 23.1 & 32.6 & 1083.0 & 1.50 \\
\hline & $3 \mathrm{~A}$ & NGHP-01-10D & $15^{\circ}$ & $51.8647^{\prime}$ & $\mathrm{N}$ & $081^{\circ}$ & $50.0709^{\prime}$ & $\mathrm{E}$ & 1050.4 & WL & 29 & 203.8 & 127.43 & 0.0 & 32.6 & 1083.0 & 77.25 \\
\hline & & & & & & & & & NGHP-01-10 TOTALS & & 61 & 418.2 & 191.78 & 228.6 & 475.6 & $\mathrm{~N} / \mathrm{A}$ & 166.75 \\
\hline GDGH12-A & 2 & NGHP-01-11A & $15^{\circ}$ & $59.4601^{\prime}$ & $\mathrm{N}$ & $081^{\circ}$ & $59.5290^{\prime}$ & $\mathrm{E}$ & 1018.0 & LWD & 0 & 0.0 & 0.00 & 200.0 & 200.0 & 1218.0 & 14.50 \\
\hline & & & & & & & & & NGHP-01-11 TOTALS & & 0 & 0.0 & 0.00 & 200.0 & 200.0 & $\mathrm{~N} / \mathrm{A}$ & 14.50 \\
\hline 1st New Site & $3 \mathrm{~A}$ & NGHP-01-12A & $15^{\circ}$ & $51.6335^{\prime}$ & $\mathrm{N}$ & $081^{\circ}$ & $50.2274^{\prime}$ & $\mathrm{E}$ & 1045.8 & & 13 & 62.1 & 50.23 & 88.8 & 150.9 & 1196.7 & 30.00 \\
\hline & & & & & & & & & NGHP-01-12 TOTALS & & 13 & 62.1 & 50.23 & 88.8 & 150.9 & $\mathrm{~N} / \mathrm{A}$ & 30.00 \\
\hline 2nd New Site & $3 \mathrm{~A}$ & NGHP-01-13A & $15^{\circ}$ & $51.7017^{\prime}$ & $\mathrm{N}$ & $081^{\circ}$ & $50.1816^{\prime}$ & $\mathrm{E}$ & 1044.0 & WL & 0 & 0.0 & 0.00 & 200.0 & 200.0 & 1244.0 & 29.00 \\
\hline & & & & & & & & & NGHP-01-13 TOTALS & & 0 & 0.0 & 0.00 & 200.0 & 200.0 & $\mathrm{~N} / \mathrm{A}$ & 29.00 \\
\hline GDGH14-A & 3B & NGHP-01-14A & $16^{\circ}$ & $03.5577^{\prime}$ & $\mathrm{N}$ & $082^{\circ}$ & $05.6218^{\prime}$ & $\mathrm{E}$ & 906.6 & WL & 23 & 180.0 & 152.76 & 0.0 & 180.0 & 1086.6 & 57.75 \\
\hline & & & & & & & & & NGHP-01-14 TOTALS & & 23 & 180.0 & 152.76 & 0.0 & 180.0 & $\mathrm{~N} / \mathrm{A}$ & 57.75 \\
\hline GDGH11 & 3B & NGHP-01-15A & $16^{\circ}$ & $05.6983^{\prime}$ & $\mathrm{N}$ & $082^{\circ}$ & $09.7467^{\prime}$ & E & 937.9 & WL & 25 & 200.0 & 170.61 & 0.0 & 200.0 & 1137.9 & 50.00 \\
\hline & & & & & & & & & NGHP-01-15 TOTALS & & 25 & 200.0 & 170.61 & 0.0 & 200.0 & $\mathrm{~N} / \mathrm{A}$ & 50.00 \\
\hline Stepout Site & 3B & NGHP-01-16A & $16^{\circ}$ & $35.5986^{\prime}$ & $\mathrm{N}$ & $082^{\circ}$ & $41.0070^{\prime}$ & $\mathrm{E}$ & 1264.5 & WL & 28 & 217.0 & 166.86 & 0.0 & 217.0 & 1481.5 & 59.50 \\
\hline & & & & & & & & & NGHP-01-16 TOTALS & & 28 & 217.0 & 166.86 & 0.0 & 217.0 & $\mathrm{~N} / \mathrm{A}$ & 59.50 \\
\hline ANGH01 & 4 & NGHP-01-17A & $10^{\circ}$ & $45.1912^{\prime}$ & $\mathrm{N}$ & $093^{\circ}$ & $06.7365^{\prime}$ & $\mathrm{E}$ & 1356.0 & & 83 & 691.6 & 563.55 & 0.0 & 691.6 & 2047.6 & 149.25 \\
\hline & 4 & NGHP-01-17B & $10^{\circ}$ & $45.1804^{\prime}$ & $\mathrm{N}$ & $093^{\circ}$ & $06.7365^{\prime}$ & $\mathrm{E}$ & 1356.0 & WL & 0 & 0.0 & 0.00 & 718.0 & 718.0 & 2074.0 & 72.25 \\
\hline & & & & & & & & & NGHP-01-17 TOTALS & & 83 & 691.6 & 563.55 & 718.00 & 1409.60 & $\mathrm{~N} / \mathrm{A}$ & 221.50 \\
\hline MNGH-REL 5 & 4 & NGHP-01-18A & $19^{\circ}$ & $09.1452^{\prime}$ & $\mathrm{N}$ & $085^{\circ}$ & $46.3758^{\prime}$ & $\mathrm{E}$ & 1386.1 & & 20 & 186.0 & 181.61 & 4.0 & 190.0 & 1576.1 & 37.50 \\
\hline & & & & & & & & & NGHP-01-18 TOTALS & & 20 & 186.0 & 181.61 & 4.0 & 190.0 & $\mathrm{~N} / \mathrm{A}$ & 37.50 \\
\hline MNGH-Gap S & 4 & NGHP-01-19A & $18^{\circ}$ & $58.6568^{\prime}$ & $\mathrm{N}$ & $085^{\circ}$ & $39.5202^{\prime}$ & $\mathrm{E}$ & 1434.2 & & 38 & 296.0 & 271.28 & 9.0 & 305.0 & 1739.2 & 71.75 \\
\hline & 4 & NGHP-01-19B & $18^{\circ}$ & $58.6532^{\prime}$ & $\mathrm{N}$ & $085^{\circ}$ & $39.5160^{\prime}$ & $\mathrm{E}$ & 1433.2 & WL & 3 & 26.3 & 25.68 & 253.7 & 280.0 & 1713.2 & 37.50 \\
\hline & & & & & & & & & NGHP-01-19 TOTALS & & 41 & 322.3 & 296.96 & 262.7 & 585.0 & $\mathrm{~N} / \mathrm{A}$ & 109.25 \\
\hline KGGH05 & 4 & NGHP-01-20A & $15^{\circ}$ & $48.5671^{\prime}$ & $\mathrm{N}$ & $081^{\circ}$ & $50.5760^{\prime}$ & $\mathrm{E}$ & 1157.9 & & 20 & 148.8 & 69.21 & 0.0 & 148.8 & 1306.7 & 33.75 \\
\hline & 4 & NGHP-01-20B & $15^{\circ}$ & $48.5709^{\prime}$ & $\mathrm{N}$ & $081^{\circ}$ & $50.5720^{\prime}$ & $\mathrm{E}$ & 1157.9 & & 4 & 38.5 & 3.67 & 148.8 & 187.3 & 1345.2 & 15.75 \\
\hline & & & & & & & & & NGHP-01-20 TOTALS & & 24 & 187.3 & 72.88 & 148.8 & 336.1 & $\mathrm{~N} / \mathrm{A}$ & 49.50 \\
\hline New FR1 & 4 & NGHP-01-21A & $15^{\circ}$ & $51.8531^{\prime}$ & $\mathrm{N}$ & $081^{\circ}$ & $50.0827^{\prime}$ & $\mathrm{E}$ & 1049.0 & & 13 & 43.1 & 30.36 & 48.4 & 91.5 & 1140.5 & 22.00 \\
\hline & 4 & NGHP-01-21B & $15^{\circ}$ & $51.8024^{\prime}$ & $\mathrm{N}$ & $081^{\circ}$ & $50.1333^{\prime}$ & $\mathrm{E}$ & 1050.0 & & 2 & 2.0 & 0.50 & 198.0 & 200.0 & 1250.0 & 33.00 \\
\hline & 4 & NGHP-01-21C & $15^{\circ}$ & $51.8492^{\prime}$ & $\mathrm{N}$ & $081^{\circ}$ & $50.0866^{\prime}$ & $\mathrm{E}$ & 1049.0 & & 4 & 4.0 & 3.88 & 74.0 & 78.0 & 1127.0 & 21.00 \\
\hline & & & & & & & & & NGHP-01-21 TOTALS & & 19 & 49.1 & 34.74 & 320.4 & 369.5 & $\mathrm{~N} / \mathrm{A}$ & 76.00 \\
\hline & & & & & $\mathrm{NC}$ & GHP-0 & 1 EXPEDIT & IION & N TOTALS: & & 494 & 3618.4 & 2847.01 & 5810.6 & 9257.8 & $\mathrm{~N} / \mathrm{A}$ & 1646.0 \\
\hline
\end{tabular}

conditions before any coring or logging could be initiated. Five sites were occupied including two that were re-occupied LWD sites and three that were new additions. All of the sites occupied during Leg 3B were located in the Krishna-Godavari Basin. The expedition was completed on Leg 4 with the drilling of two holes (one dedicated to wireline logging) at one site located in the Andaman Island area, three holes at two sites located in the Mahanadi Basin, and two holes at one site in the Krishna-Godavari Basin.

This section of the report includes a series of short descriptions of the coring and logging operations at each site established during NGHP-01. The operations at each site are also summarized in Tables 2-4. For a detailed review of the NGHP-01 operations, see 
the NGHP Expedition 01 Initial Report (Collett et al., 2008a) and the NGHP Expedition 01 Downhole Log Data Report (Collett et al., 2008b).

2.4.5.1. Leg 1. NGHP-01, Leg 1 began from Indira Dock 22, Mumbai, India, at $0700 \mathrm{~h}$ on 5 May 2006. The 246 nautical mile (NMI) transit from Mumbai to Site NGHP-01-01, in the Kerala-Konkan Basin, was completed in less than $24 \mathrm{~h}$.

Site NGHP-01-01 consisted of a single core hole (Hole NGHP-0101A). The seafloor depth for this hole was 2674.2 mbrf. Continuous coring was conducted with the standard APC/XCB coring systems to a depth of 290.0 mbsf. Six pressure cores were attempted using the PCS; only two were recovered under pressure. The last pressure core extended the hole to a depth of $290.0 \mathrm{mbsf}$. A total of six temperature measurements were acquired. Three wireline logging runs were made using the Triple-Combo, FMS-Sonic, and VSI tool strings. Operations at Site NGHP-01 ended at 0345 h on 10 May as the vessel got underway for Chennai, India. Leg 1 ended at $0624 \mathrm{~h}$ on 16 May at the Harbor Basin West Quay 4 in Chennai, India.

2.4.5.2. Leg 2. Leg 2 of NGHP-01 began at 1300 h on 19 May. This leg was completed 18.3 days later when the vessel arrived back in Chennai. Leg 2 was composed strictly of LWD drilling in the Krishna-Godavari and Mahanadi Basins off the East coast of India. No coring operations were undertaken during this leg. Twelve LWD holes were drilled at ten different sites (Table 2: Site NGHP-01-02 through Site NGHP-01-11). Leg 2 ended at 2145 h on 6 June with the first line ashore at the Harbor Basin East Quay.

2.4.5.3. Leg 3A. Leg $3 \mathrm{~A}$ began in Chennai, India (second Chennai port call) at $1745 \mathrm{~h}$ on 9 June in Harbor Basin East Quay after completing a 2.8-day re-supply port call. Eight holes were drilled on this leg at four different drill sites in the Krishna-Godavari Basin. Two sites were reoccupations of earlier sites and two were new. Leg 3A was completed 14.7 days later, when the vessel arrived back in Chennai for the third time.

This leg consisted of coring with standard APC/XCB systems (Table 2); pressure coring systems including the PCS, FPC, and HRC (Table 3); temperature measurements with the APCT-3, APCT, and DVTP (Table 4); and wireline logging with the Triple Combo, FMSSonic, and VSI tool suites.

The 203 NMI transit from Chennai to Site NGHP-01-05 was completed in just under $21 \mathrm{~h}$. Five holes were drilled at Site NGHP01-05. Two holes, NGHP-01-05A and NGHP-01-05B, were LWD holes drilled earlier on Leg 2. Holes NGHP-01-05C and NGHP-0105D were core holes, and Hole NGHP-01-05E was a drilled hole dedicated specifically for wireline logging. Operations at Site NGHP-01-05 ended at $2000 \mathrm{~h}$ on 15 June as the vessel proceeded to Site NGHP-01-10.

The 15.5 NMI transit from Site NGHP-01-05 to Site NGHP-01-10 was completed in $2.5 \mathrm{~h}$. Four holes were drilled at Site NGHP-01-10. The first hole, NGHP-01-10A, was an LWD hole drilled earlier on Leg 2. Holes NGHP-01-10B through NGHP-01-10D were cored on Leg 3A and Hole NGHP-01-10D was also wireline logged. Operations at Site NGHP-01-10 ended at 1835 h on 21 June. The move to the next site, Site NGHP-01-12, was completed at 2100 h on 21 June.

Site NGHP-01-12 was a new site (not previously LWD drilled) and consisted of a single cored hole. The original intent was to log Hole NGHP-01-12A; however, the premature abandonment of that hole precluded logging as an option. Operations at Site NGHP-01-12 were completed at 0035 h on 23 June. The move to Site NGHP-0113 was completed in $30 \mathrm{~min}$. Site NGHP-01-13 was added to the drilling schedule as a new site (not previously LWD drilled). This site was drilled for wireline logging operations. Hole NGHP-01-13A was spudded at $0130 \mathrm{~h}$ on 23 June and only a single wireline logging run was made. Operations at Site NGHP-01-13 ended at $0536 \mathrm{~h}$ on 24 June as the vessel got underway for Chennai. The 180 NMI transit from Site NGHP-01-13 to the Chennai pilot station was completed in $19.5 \mathrm{~h}$. Leg 3A ended at $0816 \mathrm{~h}$ on 25 June at Quay 3 South in Chennai, India.

2.4.5.4. Leg 3B. Leg 3B began at $1530 \mathrm{~h}$ on 28 June from the Chokhani International North Pier, after completing a 3.3 day re-supply port call. Leg 3B ended 19.0 days later at 1600 h on July 17, after completing the last hole of the leg at Site NGHP-01-16.

This leg included coring and wireline logging of eight holes at five sites (Table 2), all located in the Krishna-Godavari Basin. Two sites were reoccupations of earlier sites and three were new. The 192.0 NMI transit from Chennai to Site NGHP-01-03 was completed in $19 \mathrm{~h}$.

Three holes were drilled at Site NGHP-01-03. The first hole, NGHP-01-03A, was an LWD hole drilled earlier on Leg 2. Holes NGHP-01-03B and NGHP-01-03C were holes cored during Leg 3B. The latter was also wireline logged. Operations at Site NGHP-01-03 ended at $2045 \mathrm{~h}$ on 4 July as the vessel proceeded to Site NGHP-0107.

The 58.0-NMI transit from Site NGHP-01-03 to Site NGHP-01-07 was completed in 5.0 h. Four holes were drilled at Site NGHP-01-07. The first hole, NGHP-01-07A, was the LWD hole drilled earlier on Leg 2. Hole NGHP-01-07B was a cored hole while Holes NGHP-0107C and NGHP-01-07D were primarily designed as wireline logging holes with little or no coring attempted. Operations at Site NGHP01-07 ended at $0600 \mathrm{~h}$ on 10 July as the vessel proceeded to Site NGHP-01-14.

The 44.0 NMI transit from Site NGHP-01-07 to Site NGHP-01-14 was completed in $5.0 \mathrm{~h}$. Site NGHP-01-14 was one of the alternate sites listed in the prospectus and consisted of a single core hole (Hole NGHP-01-14A). Continuous coring was conducted with the standard APC/XCB coring systems to a depth of 180.0 mbsf. Four pressure cores were attempted using all three of the pressure coring systems available (PCS, FPC, and HRC). Three wireline logging runs were made using the Triple Combo, FMS-Sonic, and VSI tool strings. Operations at Site NGHP-01-14 ended at $2045 \mathrm{~h}$ as the vessel got underway for Site NGHP-01-15.

The 4.5 NMI move from Site NGHP-01-14 to Site NGHP-01-15 was completed in $1.3 \mathrm{~h}$. Site NGHP-01-15 was another alternate site listed in the prospectus and again consisted of a single core hole: Hole NGHP-01-15A. Continuous coring was conducted with the standard APC/XCB coring systems to a depth of 200.0 mbsf. Four pressure cores were attempted using only the IODP/TAMU PCS and the Fugro FPC pressure coring systems. Two wireline logging runs were made using the Triple-Combo and FMS-Sonic tool strings. The VSI tool was not deployed at this site due to the close proximity to Site NGHP-01-14, where a VSI survey had already been conducted. Operations at Site NGHP-01-15 ended at 0010 h on 15 July when the vessel proceeded to Site NGHP-01-16.

The 43.0 NMI transit from Site NGHP-01-15 to Site NGHP-01-16 was completed in $4.5 \mathrm{~h}$. Site NGHP-01-16 was a "step-out" site from Site NGHP-01-07. This site consisted of a single core hole (Hole NGHP-01-16A). Continuous coring was conducted with the standard APC/XCB coring systems to a depth of 217.0 mbsf. Four pressure cores were attempted using all three of the pressure coring systems available (PCS, FPC, and HRC). Two wireline logging runs were made using the Triple-Combo and FMS-Sonic tool strings. Operations at Site NGHP-01-16 and Leg 3B ended at $1600 \mathrm{~h}$ on 17 July as the vessel proceeded to Site NGHP-01-17.

2.4.5.5. Leg 4. After completing helicopter personnel exchanges and operations at Site NGHP-01-16, Leg 4 began at $1600 \mathrm{~h}$ on 17 
Table 3

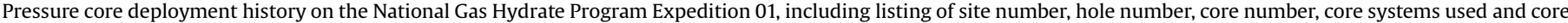

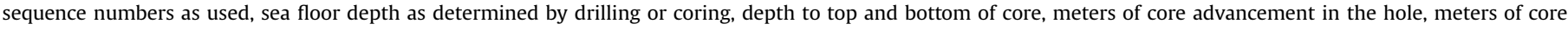

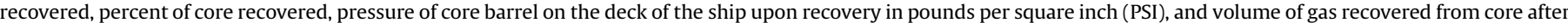

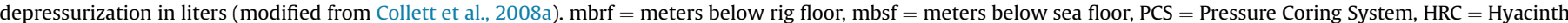
Rotary Corer, FPC = Fugro Pressure Corer, stored = notes that the core was stored and not depressurized.

\begin{tabular}{|c|c|c|c|c|c|c|c|c|c|c|c|}
\hline Site & $\begin{array}{l}\text { Hole } \\
\text { no. }\end{array}$ & $\begin{array}{l}\text { Core } \\
\text { no. }\end{array}$ & $\begin{array}{l}\text { Core } \\
\text { system \& } \\
\text { sequence }\end{array}$ & $\begin{array}{l}\text { Sea floor } \\
\text { depth } \\
\text { (mbrf) }\end{array}$ & $\begin{array}{l}\text { Depth } \\
\text { core top } \\
\text { (mbsf) }\end{array}$ & $\begin{array}{l}\text { Depth } \\
\text { core bottom } \\
\text { (mbsf) }\end{array}$ & $\begin{array}{l}\text { Meters } \\
\text { advanced }\end{array}$ & $\begin{array}{l}\text { Meters } \\
\text { of core } \\
\text { recovered }\end{array}$ & \% Recovered & $\begin{array}{l}\text { Deck pressure } \\
\text { (PSI) }\end{array}$ & Gas Rec (liters) \\
\hline \multirow[t]{6}{*}{ KKGH-01 } & \multirow[t]{6}{*}{$01 \mathrm{~A}$} & 06P & PCS-1 & 2674.2 & 42.3 & 43.3 & 1.0 & 0.91 & $91.0 \%$ & 1990 & 0.0 \\
\hline & & $12 \mathrm{P}$ & PCS-2 & 2674.2 & 91.8 & 92.8 & 1.0 & 0.00 & $0.0 \%$ & 2230 & 0.3 \\
\hline & & $20 P$ & PCS-3 & 2674.2 & 152.8 & 153.8 & 1.0 & 1.01 & $101.0 \%$ & 3730 & 0.0 \\
\hline & & $26 \mathrm{P}$ & PCS-4 & 2674.2 & 203.0 & 204.0 & 1.0 & 0.00 & $0.0 \%$ & 2010 & 0.6 \\
\hline & & $31 \mathrm{P}$ & PCS-5 & 2674.2 & 243.6 & 244.6 & 1.0 & 0.00 & $0.0 \%$ & 0 & 0.0 \\
\hline & & $37 \mathrm{P}$ & PCS-6 & 2674.2 & 289.0 & 290.0 & 1.0 & 0.00 & $0.0 \%$ & 0 & 0.0 \\
\hline \multirow[t]{12}{*}{ GDGH05-A } & \multirow[t]{5}{*}{ 03B } & $13 \mathrm{P}$ & PCS-7 & 1085.1 & 104.9 & 105.9 & 1.0 & 0.95 & $95.0 \%$ & 1014 & 1.2 \\
\hline & & $22 \mathrm{P}$ & PCS-8 & 1085.1 & 174.9 & 175.9 & 1.0 & 0.80 & $80.0 \%$ & 0 & 0.0 \\
\hline & & $23 \mathrm{Y}$ & FPC-1 & 1085.1 & 175.9 & 176.9 & 1.0 & 0.88 & $88.0 \%$ & 1544 & 5.0 \\
\hline & & $27 \mathrm{P}$ & PCS-9 & 1085.1 & 198.9 & 199.9 & 1.0 & 1.00 & $100.0 \%$ & 0 & 0.0 \\
\hline & & $28 \mathrm{E}$ & HRC-1 & 1085.1 & 199.9 & 200.9 & 1.0 & 0.00 & $0.0 \%$ & 0 & 0.0 \\
\hline & \multirow[t]{7}{*}{$03 C$} & $04 \mathrm{P}$ & PCS-10 & 1086.0 & 170.0 & 171.0 & 1.0 & 0.97 & $97.0 \%$ & 0 & 0.0 \\
\hline & & $05 Y$ & FPC-2 & 1086.0 & 175.0 & 176.0 & 1.0 & 0.87 & $87.0 \%$ & 0 & 0.0 \\
\hline & & $06 \mathrm{E}$ & HRC-2 & 1086.0 & 180.0 & 181.0 & 1.0 & 0.56 & $56.0 \%$ & 0 & 0.0 \\
\hline & & 07P & PCS-11 & 1086.0 & 184.0 & 185.0 & 1.0 & 0.95 & 0.95 & 1764 & 2.5 \\
\hline & & $08 \mathrm{Y}$ & FPC-3 & 1086.0 & 190.0 & 191.0 & 1.0 & 0.00 & $0.0 \%$ & 0 & 0.0 \\
\hline & & $09 Y$ & FPC-4 & 1086.0 & 195.0 & 196.0 & 1.0 & 0.85 & $85.0 \%$ & 0 & 0.0 \\
\hline & & $10 \mathrm{P}$ & PCS-12 & 1086.0 & 196.0 & 197.0 & 1.0 & 0.94 & $94.0 \%$ & 0 & 0.0 \\
\hline \multirow[t]{10}{*}{ KGGH02-A } & \multirow[t]{2}{*}{$05 C$} & $10 \mathrm{P}$ & PCS-13 & 957.2 & 78.3 & 79.3 & 1.0 & 0.36 & $36.0 \%$ & 426 & 1.0 \\
\hline & & $12 \mathrm{P}$ & PCS-14 & 957.2 & 86.0 & 87.0 & 1.0 & 0.85 & $85.0 \%$ & 706 & 3.0 \\
\hline & \multirow[t]{8}{*}{$05 \mathrm{D}$} & $5 \mathrm{P}$ & PCS-15 & 955.1 & 44.9 & 45.9 & 1.0 & 0.00 & $0.0 \%$ & 0 & 0.0 \\
\hline & & $6 P$ & PCS-16 & 955.1 & 76.9 & 77.9 & 1.0 & 1.00 & $100.0 \%$ & 603 & 15.0 \\
\hline & & $7 Y$ & FPC-5 & 955.1 & 84.9 & 85.9 & 1.0 & 0.88 & $88.0 \%$ & 1600 & 15.0 \\
\hline & & $8 \mathrm{E}$ & HRC-3 & 955.1 & 88.9 & 89.9 & 1.0 & 0.24 & $24.0 \%$ & 0 & 0.0 \\
\hline & & $9 \mathrm{P}$ & PCS-17 & 955.1 & 92.9 & 93.9 & 1.0 & 0.00 & $0.0 \%$ & 0 & 0.0 \\
\hline & & $10 \mathrm{E}$ & HRC-4 & 955.1 & 114.9 & 115.9 & 1.0 & 0.55 & $55.0 \%$ & 1470 & 1.0 \\
\hline & & $11 \mathrm{Y}$ & FPC-6 & 955.1 & 124.9 & 125.9 & 1.0 & 0.88 & $88.0 \%$ & 1470 & 2.0 \\
\hline & & $12 \mathrm{P}$ & PCS-18 & 955.1 & 200.0 & 201.0 & 1.0 & 1.00 & $100.0 \%$ & 103 & 2.0 \\
\hline \multirow[t]{7}{*}{ KGGH06-A } & 07B & $11 \mathrm{P}$ & PCS-19 & 1295.8 & 84.2 & 85.2 & 1.0 & 0.89 & $89.0 \%$ & 1338 & 1.0 \\
\hline & & $12 \mathrm{Y}$ & FPC-7 & 1295.8 & 85.2 & 86.2 & 1.0 & 0.90 & $90.0 \%$ & 0 & 0.0 \\
\hline & & $16 Y$ & FPC-8 & 1295.8 & 114.2 & 115.2 & 1.0 & 0.80 & $80.0 \%$ & 0 & 0.0 \\
\hline & & $21 P$ & PCS-20 & 1295.8 & 148.2 & 149.2 & 1.0 & 1.20 & $120.0 \%$ & 1470 & 4.0 \\
\hline & & $22 \mathrm{E}$ & HRC-5 & 1295.8 & 149.2 & 150.2 & 1.0 & 0.79 & $79.0 \%$ & 441 & 1.0 \\
\hline & & $28 \mathrm{P}$ & PCS-21 & 1295.8 & 193.4 & 194.4 & 1.0 & 0.92 & $92.0 \%$ & 1103 & 1.0 \\
\hline & & $29 Y$ & FPC-9 & 1295.8 & 194.4 & 195.4 & 1.0 & 0.70 & $70.0 \%$ & 0 & 0.0 \\
\hline GD-3-1 & $10 \mathrm{~B}$ & $5 P$ & PCS-22 & 1049.4 & 31.4 & 32.4 & 1.0 & 0.00 & $0.0 \%$ & 100 & 0.0 \\
\hline & & $8 Y$ & FPC-10 & 1049.4 & 50.2 & 51.2 & 1.0 & 0.90 & $90.0 \%$ & 1470 & Stored \\
\hline & & $11 \mathrm{E}$ & HRC-6 & 1049.4 & 70.0 & 71.0 & 1.0 & 0.17 & $17.0 \%$ & 0 & 0.0 \\
\hline & & $15 \mathrm{P}$ & PCS-23 & 1049.4 & 98.2 & 99.2 & 1.0 & 1.13 & $113.0 \%$ & 240 & 30.0 \\
\hline & & $18 \mathrm{Y}$ & FPC-11 & 1049.4 & 117.4 & 118.4 & 1.0 & 0.90 & $90.0 \%$ & 1735 & 52.0 \\
\hline & & $21 Y$ & FPC-12 & 1049.4 & 136.6 & 137.6 & 1.0 & 0.33 & $33.0 \%$ & 0 & 0.0 \\
\hline & & $27 \mathrm{E}$ & HRC-7 & 1049.4 & 174.1 & 175.1 & 1.0 & 0.42 & $42.0 \%$ & 0 & 0.0 \\
\hline & & $28 \mathrm{P}$ & PCS-24 & 1049.4 & 175.1 & 176.1 & 1.0 & 1.67 & $167.0 \%$ & 82 & 1.5 \\
\hline GD-3-1 & $10 \mathrm{D}$ & $6 \mathrm{P}$ & PCS-25 & 1050.4 & 40.1 & 41.1 & 1.0 & 0.29 & $29.0 \%$ & 0 & 0.0 \\
\hline & & $9 Y$ & FPC-13 & 1050.4 & 58.8 & 59.8 & 1.0 & 0.51 & $51.0 \%$ & 0 & 0.0 \\
\hline & & $12 \mathrm{E}$ & HRC-8 & 1050.4 & 77.8 & 78.8 & 1.0 & 1.09 & $109.0 \%$ & 1397 & 26.0 \\
\hline & & $15 \mathrm{P}$ & PCS-26 & 1050.4 & 97.0 & 98.0 & 1.0 & 0.92 & $92.0 \%$ & 0 & 0.0 \\
\hline & & $19 Y$ & FPC-14 & 1050.4 & 125.8 & 126.8 & 1.0 & 0.88 & $88.0 \%$ & 0 & 0.0 \\
\hline & & $22 \mathrm{E}$ & HRC-9 & 1050.4 & 145.1 & 146.1 & 1.0 & 1.05 & $105.0 \%$ & 1697 & 16.0 \\
\hline & & $25 \mathrm{P}$ & PCS-27 & 1050.4 & 164.4 & 165.4 & 1.0 & 1.22 & $122.0 \%$ & 1279 & 5.0 \\
\hline New Site & $12 \mathrm{~A}$ & $5 Y$ & FPC-15 & 1045.8 & 45.7 & 46.7 & 1.0 & 0.81 & $81.0 \%$ & 0 & 0.0 \\
\hline & & $6 \mathrm{E}$ & HRC-10 & 1045.8 & 46.7 & 47.7 & 1.0 & 1.08 & $108.0 \%$ & 1397 & 7.0 \\
\hline & & $7 P$ & PCS-28 & 1045.8 & 47.7 & 48.7 & 1.0 & 0.00 & $0.0 \%$ & 779 & 2.0 \\
\hline & & $9 Y$ & FPC-16 & 1045.8 & 93.3 & 94.3 & 1.0 & 0.88 & $88.0 \%$ & 1926 & 6.0 \\
\hline & & $10 \mathrm{E}$ & HRC-11 & 1045.8 & 94.3 & 95.3 & 1.0 & 0.00 & $0.0 \%$ & 0 & 0.0 \\
\hline & & $11 \mathrm{P}$ & PCS-29 & 1045.8 & 95.3 & 96.3 & 1.0 & 0.58 & $58.0 \%$ & 0 & 0.0 \\
\hline GDGH14-A & $14 \mathrm{~A}$ & $9 \mathrm{P}$ & PCS-30 & 906.6 & 71.0 & 72.0 & 1.0 & 0.79 & $79.0 \%$ & 0 & 0.0 \\
\hline & & $10 \mathrm{Y}$ & FPC-17 & 906.6 & 72.0 & 73.0 & 1.0 & 0.87 & $87.0 \%$ & 0 & 0.0 \\
\hline & & $14 \mathrm{P}$ & PCS-31 & 906.6 & 100.0 & 101.0 & 1.0 & 0.91 & $91.0 \%$ & 0 & 0.0 \\
\hline & & $15 \mathrm{E}$ & HRC-12 & 906.6 & 101.0 & 102.0 & 1.0 & 0.34 & $34.0 \%$ & 1250 & 1.7 \\
\hline GDGH-11 & $15 \mathrm{~A}$ & $10 \mathrm{P}$ & PCS-32 & 937.9 & 85.7 & 86.7 & 1.0 & 0.05 & $5.0 \%$ & 1029 & NA \\
\hline & & $11 \mathrm{Y}$ & FPC-18 & 937.9 & 86.7 & 87.7 & 1.0 & 0.84 & $84.0 \%$ & 1426 & 3.5 \\
\hline & & $15 \mathrm{P}$ & PCS-33 & 937.9 & 115.0 & 116.0 & 1.0 & 0.88 & $88.0 \%$ & 0 & 0.0 \\
\hline & & $16 Y$ & FPC-19 & 937.9 & 116.0 & 117.0 & 1.0 & 0.91 & $91.0 \%$ & 1470 & 2.0 \\
\hline 07 Stepout & $16 \mathrm{~A}$ & $13 \mathrm{P}$ & PCS-34 & 1264.5 & 104.2 & 105.2 & 1.0 & 0.92 & $92.0 \%$ & 853 & 1.0 \\
\hline & & $14 \mathrm{Y}$ & FPC-20 & 1264.5 & 105.2 & 106.2 & 1.0 & 0.68 & $68.0 \%$ & 0 & 0.0 \\
\hline & & $21 \mathrm{P}$ & PCS-35 & 1264.5 & 162.2 & 163.2 & 1.0 & 0.93 & $93.0 \%$ & 0 & 0.0 \\
\hline & & $22 \mathrm{E}$ & HRC-13 & 1264.5 & 163.2 & 164.2 & 1.0 & 0.43 & $43.0 \%$ & 1764 & 2.0 \\
\hline
\end{tabular}


Table 3 (continued)

\begin{tabular}{|c|c|c|c|c|c|c|c|c|c|c|c|}
\hline Site & $\begin{array}{l}\text { Hole } \\
\text { no. }\end{array}$ & $\begin{array}{l}\text { Core } \\
\text { no. }\end{array}$ & $\begin{array}{l}\text { Core } \\
\text { system \& } \\
\text { sequence }\end{array}$ & $\begin{array}{l}\text { Sea floor } \\
\text { depth } \\
\text { (mbrf) }\end{array}$ & $\begin{array}{l}\text { Depth } \\
\text { core top } \\
\text { (mbsf) }\end{array}$ & $\begin{array}{l}\text { Depth } \\
\text { core bottom } \\
\text { (mbsf) }\end{array}$ & $\begin{array}{l}\text { Meters } \\
\text { advanced }\end{array}$ & $\begin{array}{l}\text { Meters } \\
\text { of core } \\
\text { recovered }\end{array}$ & \% Recovered & $\begin{array}{l}\text { Deck pressure } \\
\text { (PSI) }\end{array}$ & Gas Rec (liters) \\
\hline \multirow[t]{10}{*}{ ANGH01 } & \multirow[t]{10}{*}{$17 \mathrm{~A}$} & $20 Y$ & FPC-21 & 1356.0 & 175.5 & 176.5 & 1.0 & 0.90 & $90.0 \%$ & 0 & 0.0 \\
\hline & & $37 \mathrm{P}$ & PCS-36 & 1356.0 & 328.0 & 329.0 & 1.0 & 0.93 & $93.0 \%$ & 794 & 1.7 \\
\hline & & $38 Y$ & FPC-22 & 1356.0 & 329.0 & 330.0 & 1.0 & 0.87 & $87.0 \%$ & 2455 & 1.9 \\
\hline & & $48 \mathrm{P}$ & PCS-37 & 1356.0 & 412.7 & 413.7 & 1.0 & 0.89 & $89.0 \%$ & 1544 & 2.5 \\
\hline & & $49 \mathrm{E}$ & HRC-14 & 1356.0 & 413.7 & 414.7 & 1.0 & 0.87 & $87.0 \%$ & 1838 & 1.9 \\
\hline & & $59 P$ & PCS-38 & 1356.0 & 499.6 & 500.6 & 1.0 & 0.60 & $60.0 \%$ & 0 & 0.0 \\
\hline & & $60 Y$ & FPC-23 & 1356.0 & 500.6 & 501.6 & 1.0 & 0.87 & $87.0 \%$ & 0 & 0.0 \\
\hline & & $70 P$ & PCS-39 & 1356.0 & 586.3 & 586.8 & 0.5 & 0.13 & $26.0 \%$ & 470 & 0.0 \\
\hline & & $71 \mathrm{E}$ & HRC-15 & 1356.0 & 586.8 & 587.8 & 1.0 & 0.00 & $0.0 \%$ & 2176 & 0.0 \\
\hline & & $81 \mathrm{E}$ & HRC-16 & 1356.0 & 672.3 & 673.3 & 1.0 & 0.42 & $42.0 \%$ & 2352 & 4.0 \\
\hline \multirow[t]{6}{*}{ MNGH - Gap Site } & \multirow[t]{6}{*}{$19 A$} & $15 \mathrm{P}$ & PCS -40 & 1434.2 & 126.0 & 127.0 & 1.0 & 0.98 & $98.0 \%$ & 1397 & 1.5 \\
\hline & & $16 \mathrm{Y}$ & FPC-24 & 1434.2 & 127.0 & 128.0 & 1.0 & 0.96 & $96.0 \%$ & 0 & 0.0 \\
\hline & & $17 \mathrm{E}$ & HRC-17 & 1434.2 & 128.0 & 129.0 & 1.0 & 0.96 & $96.0 \%$ & 1911 & 3.5 \\
\hline & & $25 \mathrm{P}$ & PCS-41 & 1434.2 & 193.3 & 194.3 & 1.0 & 0.85 & $85.0 \%$ & 0 & 0.0 \\
\hline & & $26 \mathrm{Y}$ & FPC-25 & 1434.2 & 194.3 & 195.3 & 1.0 & 0.87 & $87.0 \%$ & 0 & 0.0 \\
\hline & & $27 \mathrm{E}$ & HRC-18 & 1434.2 & 195.3 & 196.3 & 1.0 & 1.10 & $110.0 \%$ & 1911 & 8.0 \\
\hline GDGH05 & $20 \mathrm{~A}$ & $20 \mathrm{P}$ & PCS-42 & 1157.9 & 147.8 & 148.8 & 1.0 & 0.57 & $57.0 \%$ & 0 & $\mathrm{~N} / \mathrm{A}$ \\
\hline \multirow[t]{12}{*}{ New FR1 } & \multirow[t]{6}{*}{$21 \mathrm{~A}$} & $2 Y$ & FPC-26 & 1049.0 & 58.0 & 59.0 & 1.0 & 0.84 & $84.0 \%$ & 1617 & Stored \\
\hline & & $3 \mathrm{E}$ & HRC-19 & 1049.0 & 59.0 & 60.0 & 1.0 & 1.08 & $108.0 \%$ & 1544 & Stored \\
\hline & & $6 Y$ & FPC -27 & 1049.0 & 69.0 & 70.0 & 1.0 & 0.94 & $94.0 \%$ & 0 & 0.0 \\
\hline & & $7 \mathrm{E}$ & HRC-20 & 1049.0 & 70.0 & 71.0 & 1.0 & 1.09 & $109.0 \%$ & 1514 & 70.0 \\
\hline & & $10 \mathrm{Y}$ & FPC -28 & 1049.0 & 80.0 & 81.0 & 1.0 & 0.88 & $88.0 \%$ & 0 & 0.0 \\
\hline & & $11 \mathrm{E}$ & HRC-21 & 1049.0 & 81.0 & 82.0 & 1.0 & 0.47 & $47.0 \%$ & 1470 & Stored \\
\hline & \multirow[t]{2}{*}{$21 \mathrm{~B}$} & $1 \mathrm{E}$ & HRC-22 & 1050.0 & 55.0 & 56.0 & 1.0 & 0.50 & $50.0 \%$ & 1499 & Stored \\
\hline & & $2 Y$ & FPC-29 & 1050.0 & 56.0 & 57.0 & 1.0 & 0.00 & $0.0 \%$ & 0 & 0.0 \\
\hline & \multirow[t]{4}{*}{$21 C$} & $1 Y$ & FPC-30 & 1049.0 & 55.0 & 56.0 & 1.0 & 0.85 & $85.0 \%$ & 1764 & Stored \\
\hline & & $2 \mathrm{E}$ & HRC-23 & 1049.0 & 56.5 & 57.5 & 1.0 & 1.10 & $110.0 \%$ & 1544 & Stored \\
\hline & & $3 Y$ & FPC-31 & 1049.0 & 76.0 & 77.0 & 1.0 & 0.85 & $85.0 \%$ & 1646 & Stored \\
\hline & & $4 \mathrm{E}$ & HRC-24 & 1049.0 & 77.0 & 78.0 & 1.0 & 1.08 & $108.0 \%$ & 1544 & Stored \\
\hline
\end{tabular}

July. Leg 4 was completed 30.6 days later when the vessel arrived back in Chennai for the fourth and final time.

This leg consisted of coring with the standard APC/XCB systems (Table 2); pressure coring systems including the PCS, FPC, and HRC (Table 3); temperature measurements with the APCT-3 and DVTP (Table 4); and wireline logging with the Triple-Combo, FMS-Sonic, and VSI tool suites. Ten holes (Table 2) were drilled at five new drill sites located in the Andaman offshore area, the Mahanadi Basin, and the Krishna-Godavari Basin.

The 746.0 NMI transit from Site NGHP-01-16A to Site NGHP-0117 was completed in 3.0 days. Two holes were drilled at Site NGHP01-17. Hole NGHP-01-17A was the first hole of NGHP Expedition 01 drilled in the Andaman Sea and was the second deepest penetration drilled on NGHP-01. The seafloor depth for this hole was 1356.0 mbrf. Continuous coring was conducted with the standard $\mathrm{APC} / \mathrm{XCB}$ coring systems and ten pressure cores were attempted using all three pressure coring systems (PCS/FPC/HRC). The depth for this hole was $691.6 \mathrm{mbsf}$ (2047.6 mbrf) and overall core recovery was $563.55 \mathrm{~m}$ or $81.5 \%$. Eleven temperature measurements were taken in this hole. The vessel was offset $20 \mathrm{~m}$ south of Hole NGHP01-17A for the spudding of Hole NGHP-01-17B. This was a dedicated wireline logging hole and was the deepest hole drilled on NGHP-01. The hole was drilled to a total depth of $718.0 \mathrm{mbsf}$ using an XCB center bit. Three suites of wireline logging tools were deployed: the Triple-Combo and FMS-Sonic tool strings both reached the total depth of the hole. The third suite of VSI tools reached to $629.0 \mathrm{mbsf}$, or within $89.0 \mathrm{~m}$ of total depth. Operations at Site NGHP-01-17 ended at $2030 \mathrm{~h}$ on 29 July as the vessel proceeded to Site NGHP-01-18.

The 726.0 NMI transit from Site NGHP-01-17 was completed in $88.0 \mathrm{~h}$. The transit took 0.8 days longer than anticipated due to a low pressure cell that enveloped nearly the entire Bay of Bengal. Strong head winds (40-50 kt) and heavy seas/swells $(5-10 \mathrm{~m})$ were experienced during the last day of the transit, limiting the vessel's speed. The vessel reached Site NGHP-01-18 at 1234 h on 2 August.

Site NGHP-01-18 consisted of a single Hole NGHP-01-18A. After tripping the drill string to the seafloor, the vessel went into standby mode while $2.5 \mathrm{~h}$ were spent waiting on weather conditions. Coring proceeded using the APC coring system to a depth of $54.9 \mathrm{mbsf}$ (1431.5 mbrf) before coring was halted once again for another $1.5 \mathrm{~h}$ because of the weather. Coring with the APC was eventually completed to a depth of 115.9 mbsf. The XCB coring system was used to achieve the total depth of $190.0 \mathrm{mbsf}$. Operations at Site NGHP-01-18 ended at $0200 \mathrm{~h}$ on 4 August as the vessel proceeded to Site NGHP-01-19.

The 12.0 NMI transit from Site NGHP-01-18 to Site NGHP-01-19 was arduous. The large low pressure cell that enveloped a large portion of the Bay of Bengal led to strong winds (30-40 kt) and heavy seas/swells (5-10 m) throughout the transit. Originally, only one hole was scheduled for drilling at Site NGHP-01-19; however, due to hole instability problems in Hole NGHP-01-19A, a separate dedicated wireline logging hole, NGHP-01-19B, was required. Continuous coring was conducted in Hole NGHP-01-19A with the standard APC/XCB coring systems to a depth of $305.0 \mathrm{mbsf}$. There were six pressure cores attempted in Hole NGHP-01-19A using all three of the pressure-coring systems available (PCS, FPC, and HRC). Only one wireline logging run was made with the Triple-Combo tool string; however this string was unable to pass below 145.8 mbsf. Logs were obtained above this point and the decision was made to drill a dedicated logging hole to complete the wireline logging program.

The vessel was offset $10 \mathrm{~m}$ southwest of Hole NGHP-01-19A for the spudding of Hole NGHP-01-19B. Although this was to be a drilled hole dedicated to recovering a full suite of wireline logs, it was also used as an opportunity to recover three shallow APC cores for future high-resolution sedimentological studies. Three suites of wireline logging tools were deployed. Operations at Site NGHP-01- 
Table 4

Downhole temperature tool deployment history on the National Gas Hydrate Program Expedition 01, including listing of site number, hole number, associated core number, temperature system used to make measurement, and measurement point depth below seafloor (modified from Collett et al., 2008a). mbsf = meters below seafloor, APCT $=$ Advanced Piston Corer Temperature Probe, APCT-3 = Advanced Piston Corer Temperature Probe (third generation), DVTP $=$ Davis-Villinger Temperature Pressure probe.

\begin{tabular}{|c|c|c|c|c|}
\hline Site & Hole no. & Core no. & Temperature system & Depth (mbsf) \\
\hline \multirow[t]{6}{*}{ NGHP-01-01 } & \multirow[t]{6}{*}{$01 \mathrm{~A}$} & $4 \mathrm{H}$ & APCT & 42.3 \\
\hline & & $7 \mathrm{H}$ & APCT & 63.3 \\
\hline & & $9 \mathrm{H}$ & APCT & 82.3 \\
\hline & & $13 \mathrm{H}$ & APCT & 112.8 \\
\hline & & - & DVTP & 164.5 \\
\hline & & - & DVTP & 214.6 \\
\hline \multirow[t]{6}{*}{ NGHP-01-03 } & \multirow[t]{6}{*}{ 03B } & $3 \mathrm{H}$ & ACPT-3 & 33.9 \\
\hline & & $5 \mathrm{H}$ & ACPT-3 & 52.9 \\
\hline & & $7 \mathrm{H}$ & ACPT-3 & 71.7 \\
\hline & & - & DVTP & 151.7 \\
\hline & & - & DVTP & 220.3 \\
\hline & & - & DVTP & 300.0 \\
\hline \multirow[t]{7}{*}{ NGHP-01-05 } & \multirow[t]{5}{*}{$05 \mathrm{C}$} & $3 \mathrm{H}$ & APCT-3 & 30.8 \\
\hline & & $4 \mathrm{H}$ & APCT & 40.3 \\
\hline & & $6 \mathrm{H}$ & АРCТ-3 & 59.3 \\
\hline & & - & DVTP & 115.8 \\
\hline & & - & DVTP & 200.0 \\
\hline & \multirow[t]{2}{*}{ 05D } & $3 \mathrm{H}$ & APCT & 35.9 \\
\hline & & $4 \mathrm{H}$ & APCT & 44.9 \\
\hline \multirow[t]{6}{*}{ NGHP-01-07 } & \multirow[t]{5}{*}{ 07B } & $3 \mathrm{H}$ & APCT-3 & 31.2 \\
\hline & & $5 \mathrm{H}$ & АРCT-3 & 50.2 \\
\hline & & $7 \mathrm{H}$ & АРCТ -3 & 69.2 \\
\hline & & - & DVTP & 115.2 \\
\hline & & - & DVTP & 174.2 \\
\hline & 07D & - & DVTP & 260.0 \\
\hline \multirow[t]{6}{*}{ NGHP-01-10 } & \multirow[t]{3}{*}{$10 \mathrm{~B}$} & - & DVTP & 32.4 \\
\hline & & - & DVTP & 99.2 \\
\hline & & - & DVTP & 176.1 \\
\hline & \multirow[t]{3}{*}{$10 \mathrm{D}$} & - & DVTP & 59.8 \\
\hline & & - & DVTP & 126.8 \\
\hline & & - & DVTP & 203.8 \\
\hline NGHP-01-14 & $14 \mathrm{~A}$ & $3 \mathrm{H}$ & АРCТ-3 & 33.0 \\
\hline & & $5 \mathrm{H}$ & АРCТ-3 & 52.0 \\
\hline & & $7 \mathrm{H}$ & АРCТ -3 & 71.0 \\
\hline & & - & DVTP & 150.0 \\
\hline & & - & DVTP & 169.2 \\
\hline & & - & DVTP & 180.0 \\
\hline NGHP-01-15 & $15 \mathrm{~A}$ & $3 \mathrm{H}$ & АРCТ-3 & 38.1 \\
\hline & & $5 \mathrm{H}$ & АРCТ-3 & 57.1 \\
\hline & & $7 \mathrm{H}$ & АРCТ -3 & 76.1 \\
\hline & & - & DVTP & 136.2 \\
\hline & & - & DVTP & 193.8 \\
\hline NGHP-01-16 & $16 \mathrm{~A}$ & $3 \mathrm{H}$ & АРCT-3 & 35.5 \\
\hline & & $5 \mathrm{H}$ & АРCТ-3 & 54.5 \\
\hline & & $7 \mathrm{H}$ & АРCT-3 & 65.8 \\
\hline & & - & DVTP & 125.4 \\
\hline & & - & DVTP & 144.6 \\
\hline & & - & DVTP & 182.6 \\
\hline & & - & DVTP & 209.8 \\
\hline & & - & DVTP & 217.0 \\
\hline NGHP-01-17 & $17 \mathrm{~A}$ & $3 \mathrm{H}$ & АРCT-3 & 33.0 \\
\hline & & $5 \mathrm{H}$ & АРCT-3 & 52.0 \\
\hline & & $7 \mathrm{H}$ & АРCТ -3 & 71.0 \\
\hline & & - & DVTP & 203.9 \\
\hline & & - & DVTP & 330.0 \\
\hline & & - & DVTP & 366.8 \\
\hline & & - & DVTP & 414.7 \\
\hline & & - & DVTP & 501.6 \\
\hline & & - & DVTP & 587.8 \\
\hline & & - & DVTP & 625.0 \\
\hline & & - & DVTP & 662.7 \\
\hline NGHP-01-18 & $18 \mathrm{~A}$ & $3 \mathrm{H}$ & АРCT-3 & 35.9 \\
\hline & & $5 \mathrm{H}$ & АРCТ-3 & 54.9 \\
\hline & & $7 \mathrm{H}$ & АРCТ-3 & 73.9 \\
\hline & & - & DVTP & 135.2 \\
\hline & & - & DVTP & 190.0 \\
\hline NGHP-01-19 & $19 A$ & $3 \mathrm{H}$ & АРCТ-3 & 33.8 \\
\hline & & $5 \mathrm{H}$ & АРCТ-3 & 52.8 \\
\hline
\end{tabular}

Table 4 (continued)

\begin{tabular}{llllc}
\hline Site & Hole no. & Core no. & Temperature system & Depth (mbsf) \\
\hline & & $7 \mathrm{H}$ & APCT-3 & 66.3 \\
& & - & DVTP & 117.4 \\
& & - & DVTP & 157.9 \\
& & - & DVTP & 196.3 \\
& & - & DVTP & 300.0 \\
NGHP-01-20 & $20 \mathrm{~A}$ & - & DVTP & 305.0 \\
& & $3 \mathrm{H}$ & APCT-3 & 32.5 \\
& & - & DVTP & 70.9 \\
\hline
\end{tabular}

19 ended at $0354 \mathrm{~h}$ on 9 August as the vessel proceeded for a return visit to Site NGHP-01-18.

The vessel transited north to the previously occupied Site NGHP01-18 to recover the positioning beacon that was left at the site earlier. After recovering the beacon, the vessel departed for Site NGHP-01-20 at $0700 \mathrm{~h}$ on 9 August. The 301 NMI transit from Site NGHP-01-18 to Site NGHP-01-20 was completed in $32.3 \mathrm{~h}$.

Originally, only one hole was scheduled for drilling at Site NGHP-01-20. Severe hole instability was experienced in Hole NGHP-01-20A, which required a second hole to be drilled at this site (Hole NGHP-01-20B). Continuous coring was conducted in Hole NGHP-01-20A with the standard APC/XCB coring systems to a depth of $148.8 \mathrm{mbsf}$. There was only one pressure core attempted in this hole with the PCS, which recovered a small amount of core without pressure. Several attempts were made during the coring operation to condition and stabilize the hole. The decision was made to drill another hole hoping conditions would be better in the new hole. The vessel was offset $10 \mathrm{~m}$ northwest of Hole NGHP-0120A for the spudding of Hole NGHP-01-20B. This hole was abandoned at a depth of $187.3 \mathrm{mbsf}$ for the same reasons as Hole NGHP01-20A; poor core quality and recovery, generally poor hole conditions, and packing-off of the hole annulus. Operations at Site NGHP-01-20 ended at $1630 \mathrm{~h}$ on 12 August as the vessel proceeded to Site NGHP-01-21.

The 3.3 NMI transit from Site NGHP-01-20 to Site NGHP-01-21 was made in 1.0 h. Site NGHP-01-21 was a re-occupation of Site NGHP-01-10 (drilled earlier on Leg 3A). Site NGHP-01-10 had provided the richest accumulation of gas hydrate observed during the expedition. Therefore, Site NGHP-01-21 was established in close proximity to Site NGHP-01-10, to obtain additional samples of gas hydrate for preservation, and to enable further delineation of the Site NGHP-01-10 accumulation. Ultimately there were three cored holes established at this site.

Operations at Site NGHP-01-21 were completed at 2130 h on 15 August as the vessel proceeded to Chennai. The 188.0 NMI transit from Site NGHP-01-21 to Chennai, was completed in $21.2 \mathrm{~h}$. At $1845 \mathrm{~h}$ on 16 August the vessel arrived at the Chennai Harbour anchorage. At $0340 \mathrm{~h}$ the following morning the vessel was allowed to enter Harbour Basin (Ambedkar Dock) at 0510 h on 17 August 2006, ending NGHP-01, Leg 4 operations.

The primary activity for this fourth and final Chennai port call was the official demobilization of the vessel from the NGHP-01 gas hydrate project. All standard cores and all of the gas hydrate samples destined for India were offloaded (389 core boxes and 102 boxes of samples). In addition, 35 hydrate samples preserved in three liquid-nitrogen dewars were offloaded. DGH arranged for transportation by refrigerated truck from Chennai to Mumbai (where the cores and samples were to be stored). The vessel departed India on 19 August from the North Quay, Chennai at $1912 \mathrm{~h}$; ending the NGHP-01 expedition.

\subsubsection{NGHP-01 gas hydrate occurrence}

One of the specific sets of objectives of this expedition was to test gas hydrate formation models and constrain model 


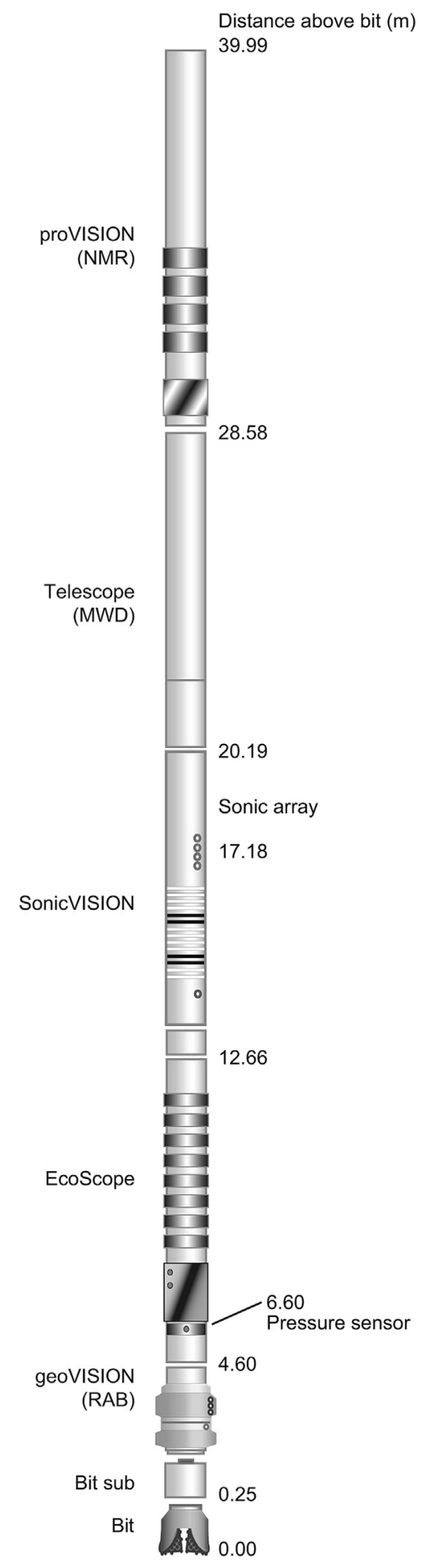

Figure 5. Bottom-hole assembly (BHA) used for logging-while-drilling operations during the National Gas Hydrate Program Expedition 01 (modified from Collett et al., 2008a, 2008b).

parameters, especially those that account for the formation of concentrated gas hydrate occurrences. The necessary data for characterizing the occurrence of in situ gas hydrate, such as interstitial water chlorinities, core-derived gas chemistry, core physical properties, IR thermal images of the recovered core, pressure core analysis, and downhole measured logging data (LWD and/or conventional wireline log data) were obtained from most of the research sites established during NGHP Expedition 01 (Fig. 3, Tables 1 and 2). Except for Site NGHP-01-01, most of the sites established during NGHP Expedition 01, yielded some evidence for the occurrence of gas hydrate. The inferred in situ concentration of gas hydrate varied substantially from site to site.

The evidence for the occurrence of gas hydrate at each of the sites established during NGHP-01 is summarized in this section of the report. The seismic, core, and well log evidence for the occurrence of gas hydrates has been tabulated in Table 5 for each of the drill sites established during NGHP-01, which links directly to a series of detailed "site summaries" included in Kumar et al., (this issue).

2.4.6.1. Seismic data analysis. The presence of gas hydrate in marine environments has been historically inferred mainly from anomalous seismic reflectors that most commonly derive from physical sediment changes associated with the transition from gas hydrate to free-gas at the base of the gas hydrate stability zone (BGHSZ) (Shipley et al., 1979). This reflector is commonly referred to as a bottom-simulating reflector or BSR as it is controlled primarily by depth-dependent phenomena (primarily temperature and pressure) and therefore parallels the seafloor without regard to the orientation of stratigraphic bedding. In less deformed areas, however, the BSR can be conformable to bedding and can be difficult to identify. BSRs have been mapped to depths as great as 200 to 400 mbsf along western and eastern margins of the Indian peninsula; whereas in the Andaman Sea BSRs have been identified at depths as great as 600 mbsf (Ramana et al., 2006).

In 2004, an extensive seismic data acquisition program followed by a multi-well drilling program in the Japan Nankai Trough (Tsuji et al., 2009) discovered no strong correlation between the occurrence of highly-concentrated gas hydrate accumulations in sandrich reservoirs and the presence or nature of BSRs. Based on the 2004 Nankai Trough drilling results, Saeki et al. (2008) recommended a gas hydrate exploration approach that integrates the evaluation of BSRs with other features, including geologic and geophysical indicators of gas hydrate in the form of strong positive seismic amplitude reflectors and elevated internal velocities, and geologic interpretation of sand-rich depositional facies. Exploration approaches that de-emphasizes BSRs as the primary indicators of gas hydrate accumulations in lieu of integrated geological and geophysical evaluations to identify and quantitatively assess sandhosted gas hydrate occurrences (Shelander et al., 2010, 2012) have been further developed and used in subsequent gas hydrate prospecting efforts, notably in the Gulf of Mexico (see papers included in Collett and Boswell, 2012).

For the NGHP-01 pre-drill site review process, there was a strong reliance on the use of BSRs as an indicator of gas hydrates. However, during the NGHP-01 expedition, the science team on the ship continued to adapt the drill site review and selection process to take advantage of the evolving understanding of the seismic manifestation of concentrated gas hydrate occurrences. In all but one of the sites established during NGHP-01 (i.e., NGHP-01-01), the pre-expedition interpreted BSRs were proven to be closely linked with the predicted base of the gas hydrate stability zone. For the most part, however, the concentration of gas hydrate and/or freegas associated with the drilled BSRs was shown to be low. Preexpedition concerns about the unusually deep nature and physical appearance of the BSR associated with Site NGHP-01-17 in the Andaman Sea was shown to be a product of a very low geothermal gradient. However, a suspect BSR interpreted for the Kerala-Konkan Basin was shown to be unrelated to gas hydrates and indicative of a lithologic change within the stratigraphic section. The pre-drill site seismic analysis of the Mahanadi Basin had defined the BGHSZ to be the top of a series of highly reflective 


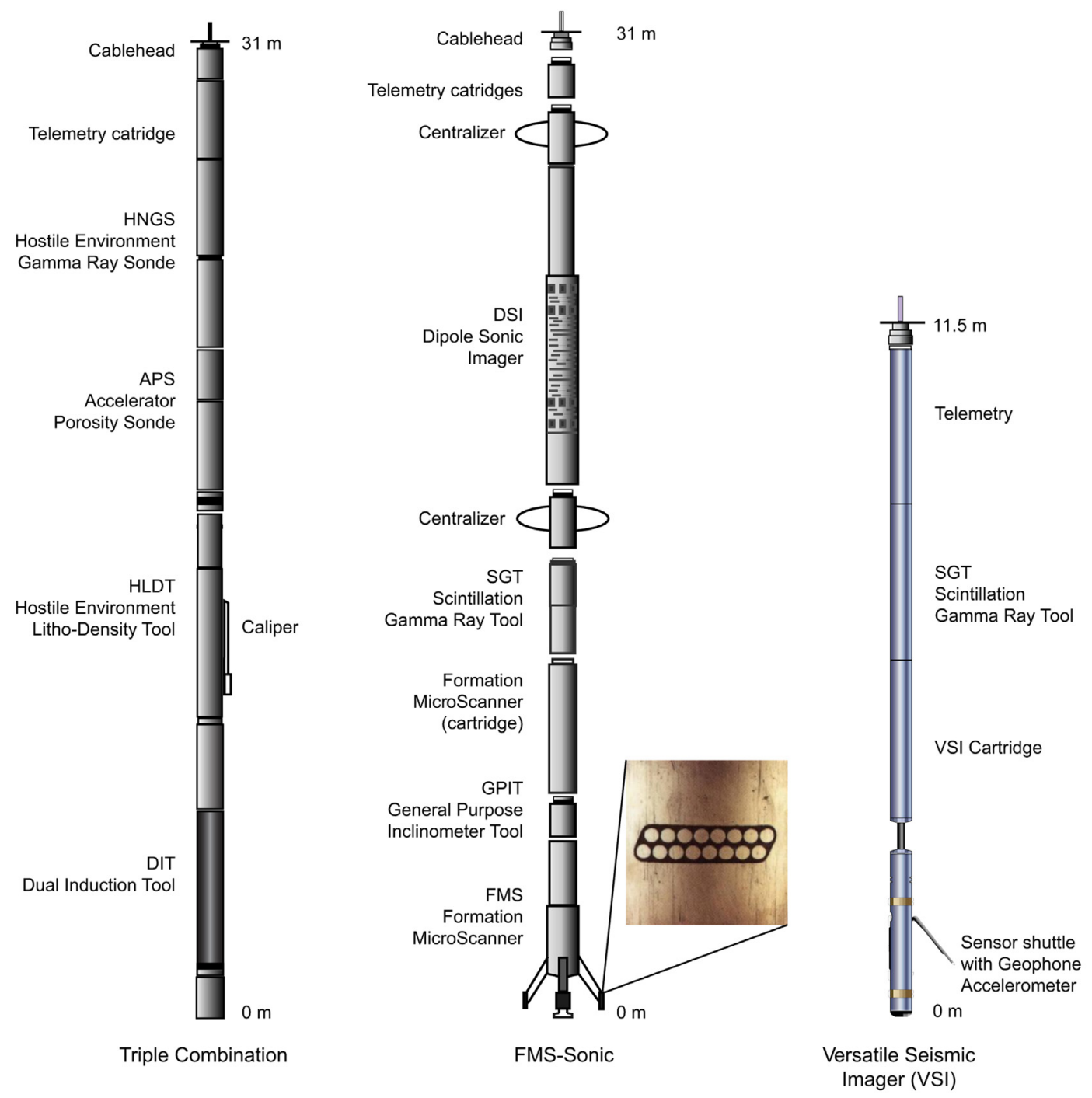

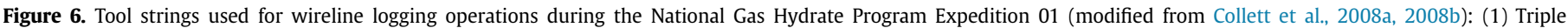
Combination; (2) FMS-Sonic, and (3) Vertical Seismic Imager.

channelized canyon-like features. Sites NGHP-01-18 and NGHP-0119 were designed as linked sites that targeted one of the seismic inferred canyon-like features in the Mahanadi Basin, which supported the interpretation that the highly reflective features did mark the top of a "reservoir controlled" low-saturation free-gas accumulations that were overlain by an equally low-saturation gas hydrate accumulation.

In several instances, pre-drill site surveys also revealed direct seismic indicators of gas hydrate in the form of strong positive seismic reflectors (i.e., high-velocity stratigraphic units). One of the best examples was documented at Site NGHP-01-07 in the Krishna-Godavari Basin, where two distinct seismic reflections (depths of $\sim 70 \mathrm{mbsf}$ and $\sim 130 \mathrm{mbsf}$ ) were identified within the sediment package overlying the BSR (Kumar et al., this issue). Pre-coring LWD and core data (Table 5: Core IR, interstitial water chemistry anomalies, and pressure-core-derived gas volumes) confirmed the occurrence of gas hydrate at the same depths of the two anomalous seismic reflections identified in the pre-drill site review.

Drilling and coring results at the NGHP-01-18 and NGHP-01-19 Mahanadi Basin sites also provided additional insight to the use and limits of seismic data as a direct indicator of gas hydrate. Lee et al. (2009) showed that sedimentary sections with relatively low free- gas saturations $(<20 \%)$ are usually characterized by strong negative amplitude seismic reflectors. A similar sedimentary section with relatively low gas hydrate saturations $(<20 \%)$ would have little to no seismic response. This relationship explains the seismic response of the canyon-like features in the Mahanadi Basin; the free-gas portion of the reservoir can be easily seen on the available seismic lines, but the gas hydrate-bearing portion of the canyon sediment fill exhibits no apparent seismic response.

2.4.6.2. Core temperature analysis. A variety of approaches have been used to determine gas hydrate occurrence through observation of core temperatures to take advantage of the thermal impact of gas hydrate dissociation. One of the early systematic approaches, applied during ODP Leg 164, used an array of digitally recorded thermocouple probes (Paull et al., 1996). Since those early efforts, digital thermal infrared (IR) imaging cameras that permit quantitative temperature estimates of recovered sediment cores have become available. Systematic IR thermal imaging of the surface of recovered core liners was first fully implemented during ODP Leg 204 (Tréhu et al., 2004).

For IODP Expedition 311 and NGHP-01, an improved IR track system was used where the IR camera traveled along the core on a 
Table 5

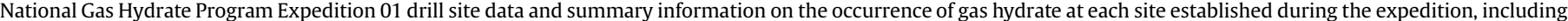

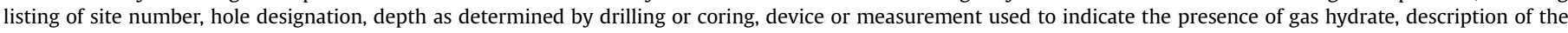

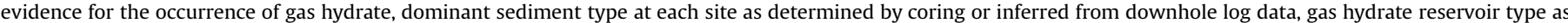

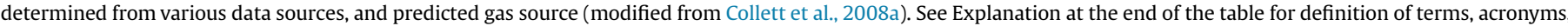
and measurement units used in this table.

\begin{tabular}{|c|c|c|c|c|}
\hline Site & Hole & Depth (mbsf) & Indicator & Description \\
\hline \multicolumn{5}{|c|}{$\begin{array}{l}\text { Krishna-Godavari Basin } \\
\text { NGHP-01-02 }\end{array}$} \\
\hline & $\bar{A}$ & $\begin{array}{l}170 \\
70-170\end{array}$ & $\begin{array}{l}\text { BSR } \\
\text { Well logs (resistivity) }\end{array}$ & $\begin{array}{l}\text { Slightly elevated resistivity ( } 1 \text { - } \\
2 \Omega-\mathrm{m} \text { ) over background; } \\
\text { Archie } \mathrm{Sh}<10 \%\end{array}$ \\
\hline
\end{tabular}

Dominant Gas hydrate Gas source

sediment type reservoir type

No core Possible No core

fracture-filling

and/or pore-

filling

-m) over background;

Archie Sh $<10 \%$

$\begin{array}{lll}\text { Clay with } & \text { Possible } & \text { Microbial } \\ \text { limited silt/ } & \text { fracture-filling } & \\ \text { sand beds } & \text { and/or pore- } & \\ & \text { filling }\end{array}$

BSR

Core water chemistry Slightly depleted $\mathrm{Cl}^{-}$

$\left(\mathrm{Cl}^{-}\right)$

concentrations

Core (pressure core) gas $\quad<1.0 \% \mathrm{Sh}$

volume

Core (pressure core) gas $\quad<1.0 \% \mathrm{Sh}$

volume

A $\quad 0-209$

Well logs (resistivity) Thinly bedded, slightly elevated resistivity $(\sim 0.5 \Omega-\mathrm{m})$ over background; archie $\mathrm{Sh}<20 \%$

NGHP-01-04

$\begin{array}{ll}- & 182 \\ \text { A } & 80-100\end{array}$

BSR

Well logs (resistivity)

Fracture, slightly elevated resistivity $(\sim 0.5 \Omega-\mathrm{m})$ over background; a Archie Sh $<25 \%$

NGHP-01-05

$\begin{array}{ll}- & 125 \\ \text { C/D } & 70-125 \\ & \\ \text { D } & 114.9,124.9 \\ & \\ \text { C/D } & 76.9,78.3,84.9, \\ & 86.0 \\ \text { A } & 55-94\end{array}$

IR associated core Depleted $\mathrm{Cl}^{-}$concentrations water chemistry $\left(\mathrm{Cl}^{-}\right) \quad$ with depth to the BSR anomaly

Core (pressure core) gas $\quad<1.0 \% \mathrm{Sh}$ volume

volume

Well logs (resistivity)

Series of gas hydrate filled fractures, elevated resistivity (2.0-5.0 $\Omega$-m) over

background; a Archie Sh ranging from 20 to $70 \%$

Well logs (resistivity)

feries of gas hydrate filled fractures, elevated resistivity (2.0-5.0 $\Omega$-m) over background; archie Sh ranging from 20 to $70 \%$

NGHP-01-06

$\bar{A}$
210

137-148

BSR

Well logs (resistivity)

Series of gas hydrate filled fractures, slightly elevated resistivity (0.5-1.0 $\Omega$-m) over background; a Archie Sh $<25 \%$

Clay with limited silt sand beds
Fracture-filling No core with possible pore-filling

Clay with limited silt/ sand beds

Fracture-filling with possible pore-filling

NGHP-01-07

$\begin{array}{ll}- & 188 \\ - & 70,130 \\ \text { B/D } & 15-188 \\ & \\ \text { B } & 148.2\end{array}$

BSR

High amplitude seismic reflector above BGHSZ

IR associated core water chemistry $\left(\mathrm{Cl}^{-}\right)$ anomaly

Core (pressure core) gas volume
No core

Fracture-filling with possible pore-filling
Depleted and elevated $\mathrm{Cl}^{-}$ concentrations with depth to the BSR 
Table 5 (continued)

\begin{tabular}{|c|c|c|c|c|c|c|c|}
\hline Site & Hole & Depth (mbsf) & Indicator & Description & $\begin{array}{l}\text { Dominant } \\
\text { sediment type }\end{array}$ & $\begin{array}{l}\text { Gas hydrate } \\
\text { reservoir type }\end{array}$ & Gas source \\
\hline & A & $70-90$ & Well logs (resistivity) & $\begin{array}{l}\text { Series of gas hydrate filled } \\
\text { fractures, elevated resistivity } \\
(0.5-2.0 \Omega-m) \text { over } \\
\text { background; archie } \text { Sh }<25 \%\end{array}$ & & & \\
\hline & A & $130-150$ & Well logs (resistivity) & $\begin{array}{l}\text { Series of gas hydrate filled } \\
\text { fractures, elevated resistivity } \\
(0.5-2.0 \Omega-m) \text { over } \\
\text { background; a Archie Sh }<25 \%\end{array}$ & & & \\
\hline \multirow{8}{*}{$\begin{array}{l}\text { NGHP-01-10 } \\
\text { NGHP-01-12 } \\
\text { NGHP-01-13 } \\
\text { NGHP-01-21 }\end{array}$} & & & & & Clay with & Fracture-filling & Microbial \\
\hline & & & & & limited silt/ & with possible & \\
\hline & & & & & sand beds & pore-filling & \\
\hline & & & & & & & \\
\hline & - & 160 & BSR & & & & \\
\hline & $\begin{array}{l}10 \mathrm{~B} / 10 \mathrm{D} / 12 \mathrm{~A} / \\
21 \mathrm{~A}\end{array}$ & $26-160$ & $\begin{array}{l}\text { IR associated core } \\
\text { water chemistry }\left(\mathrm{Cl}^{-}\right) \\
\text {anomaly }\end{array}$ & $\begin{array}{l}\text { Depleted and elevated } \mathrm{Cl}^{-} \\
\text {concentrations with depth to } \\
\text { the BSR }\end{array}$ & & & \\
\hline & $\begin{array}{l}10 \mathrm{~B} / 10 \mathrm{D} / 12 \mathrm{~A} / \\
21 \mathrm{~A}\end{array}$ & $\begin{array}{l}46.7,47.4,70.0 \\
77.8,93.3,98.2 \\
117.4,145.1 \\
164.4\end{array}$ & $\begin{array}{l}\text { Core (pressure core) gas } \\
\text { volume }\end{array}$ & $2.0-31.5 \% \mathrm{Sh}$ & & & \\
\hline & $10 \mathrm{~A}$ & $27-160$ & Well logs (resistivity) & 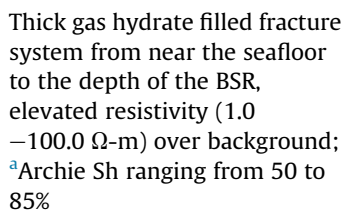 & & & \\
\hline \multirow[t]{4}{*}{ NGHP-01-11 } & & & & & No core & $\begin{array}{l}\text { Fracture-filling } \\
\text { with possible } \\
\text { pore-filling }\end{array}$ & No core \\
\hline & - & 150 & BSR & & & & \\
\hline & A & $95-113$ & Well logs (resistivity) & $\begin{array}{l}\text { Series of gas hydrate filled } \\
\text { fractures, elevated resistivity } \\
(0.5-1.5 \Omega-m) \text { over } \\
\text { background; a Archie Sh }<35 \%\end{array}$ & & & \\
\hline & A & $144-146$ & Well logs (resistivity) & $\begin{array}{l}\text { Gas hydrate filled fracture, } \\
\text { elevated resistivity }(0.5-3.5 \Omega- \\
\text { m) over background; }{ }^{\text {Aarchie }} \text { Sh } \\
<35 \%\end{array}$ & & & \\
\hline \multirow[t]{6}{*}{ NGHP-01-14 } & & & & & $\begin{array}{l}\text { Clay with } \\
\text { slightly more } \\
\text { abundant silt/ } \\
\text { sand beds }\end{array}$ & $\begin{array}{l}\text { Possible pore- } \\
\text { filling (highly } \\
\text { disseminated } \\
\text { gas hydrate) }\end{array}$ & Microbial \\
\hline & - & 109 & BSR & & & & \\
\hline & A & 20-109 & $\begin{array}{l}\text { IR associated core } \\
\text { water chemistry }\left(\mathrm{Cl}^{-}\right) \\
\text {anomaly }\end{array}$ & $\begin{array}{l}\text { Depleted } \mathrm{Cl}^{-} \text {concentrations } \\
\text { with depth to the BSR }\end{array}$ & & & \\
\hline & A & 101 & $\begin{array}{l}\text { Core (pressure core) gas } \\
\text { volume }\end{array}$ & $10.7 \% \mathrm{Sh}$ & & & \\
\hline & A & $67-72$ & Well logs (resistivity) & $\begin{array}{l}\text { Thinly bedded, slightly elevated } \\
\text { resistivity }(0.5-1.0 \Omega-\mathrm{m}) \text { over } \\
\text { background; Archie Sh }<20 \%\end{array}$ & & & \\
\hline & A & $82-87$ & Well logs (resistivity) & $\begin{array}{l}\text { Thinly bedded, slightly elevated } \\
\text { resistivity }(\sim 0.5 \Omega-\mathrm{m}) \text { over } \\
\text { background; Archie } \text { Sh }<20 \%\end{array}$ & & & \\
\hline \multirow[t]{5}{*}{ NGHP-01-15 } & & & & & $\begin{array}{l}\text { Clay with more } \\
\text { abundant silt/ } \\
\text { sand beds }\end{array}$ & $\begin{array}{l}\text { Pore-filling } \\
\text { (ranging from } \\
\text { disseminated to } \\
\text { one highly } \\
\text { saturated gas } \\
\text { hydrate sand } \\
\text { bed) }\end{array}$ & Microbial \\
\hline & - & 126 & BSR & & & & \\
\hline & A & $60-90$ & $\begin{array}{l}\text { IR associated core } \\
\text { water chemistry }\left(\mathrm{Cl}^{-}\right) \\
\text {anomaly }\end{array}$ & $\begin{array}{l}\text { Depleted } \mathrm{Cl}^{-} \text {concentrations } \\
\text { associated with disseminated } \\
\text { gas hydrate and one distinct } \\
\text { sand bed ( } 78.63-79.13 \mathrm{mbsf})\end{array}$ & & & \\
\hline & A & 86.7 & $\begin{array}{l}\text { Core (pressure core) gas } \\
\text { volume }\end{array}$ & $16.1 \% \mathrm{Sh}$ & & & \\
\hline & A & $75-81$ & Well logs (resistivity) & $\begin{array}{l}\text { Interbedded clay/silt/sand } \\
\text { section, elevated resistivity }(0.5 \\
-5.0 \Omega-\mathrm{m}) \text { over background; }\end{array}$ & & & \\
\hline
\end{tabular}


Table 5 (continued)

\begin{tabular}{|c|c|c|c|c|}
\hline Site & Hole & Depth (mbsf) & Indicator & Description \\
\hline & A & $90-110$ & Well logs (resistivity) & $\begin{array}{l}\text { Archie Sh ranging from } 25 \text { to } \\
50 \% \\
\text { Interbedded clay/silt/sand } \\
\text { section, elevated resistivity ( } 0.5 \\
-1.0 \Omega-\mathrm{m} \text { ) over background; } \\
\text { Archie Sh ranging from } 25 \text { to } \\
50 \%\end{array}$ \\
\hline
\end{tabular}

NGHP-01-16

$\begin{array}{llll}- & 170 & \text { BSR } & \\ \text { A } & 85-170 & \begin{array}{l}\text { IR associated core } \\ \text { water chemistry }\left(\mathrm{Cl}^{-}\right) \\ \text {anomaly } \\ \text { Core (pressure core) gas } \\ \text { volume }\end{array} & \begin{array}{l}\text { Depleted } \mathrm{Cl}^{-} \text {concentrations } \\ \text { with depth to the BSR }\end{array} \\ \text { A } & 163.1 & \text { Well logs (resistivity) } & \begin{array}{l}\text { Thinly bedded, slightly elevated } \\ \text { resistivity }(0.5-3.0 \Omega \text {-m) over } \\ \text { background; Archie Sh ranging } \\ \text { from } 10 \text { to } 50 \%\end{array} \\ & 90-155 & & \end{array}$

NGHP-01-20 from 10 to $50 \%$

$\begin{array}{ll}\begin{array}{ll}\text { Clay with more } \\ \text { abundant silt/ }\end{array} & \begin{array}{l}\text { Possible pore- } \\ \text { filling }\end{array} \\ \text { sand beds } & \begin{array}{l}\text { (disseminated } \\ \text { gas hydrate) }\end{array}\end{array}$

Dominant Gas hydrate Gas source
sediment type reservoir type

source Archie Sh ranging from 25 to $50 \%$ gas hydrate)

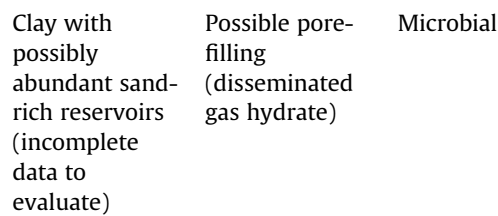

Local, discontinuous BSR?

IR associated core $\quad$ Depleted $\mathrm{Cl}^{-}$concentrations water chemistry $\left(\mathrm{Cl}^{-}\right)$with depth to the bottom of the anomaly

B hole. Possible significant sand reservoirs based on core recovery problems.

$\begin{array}{ll}\text { No core } & \begin{array}{l}\text { Possible pore- } \\ \text { filling and/or }\end{array}\end{array}$

BSR

Well logs (resistivity) Slightly elevated resistivity ( $0.2 \Omega-\mathrm{m}$ ) over background Archie Sh $<10 \%$ fracture-filling$$
\text { Archie Sh }<10 \%
$$

BSR

Well logs (resistivity) Slightly elevated resistivity

( $0.5 \Omega-\mathrm{m})$ over background; Archie Sh $<10 \%$

No core

Possible pore- $\quad$ No core filling and/or fracture-filling

\begin{tabular}{|c|c|}
\hline $\begin{array}{l}\text { Clay with } \\
\text { limited silt/ }\end{array}$ & $\begin{array}{l}\text { Possible pore- } \\
\text { filling }\end{array}$ \\
\hline
\end{tabular}
sand beds

BSR (top of highreflectivity zone anomaly

NGHP-01-19

$\begin{array}{ll}- & 205 \\ \text { A } & 125-130 \\ \text { A } & 170-205 \\ \text { A } & 128.0,195.3 \\ \text { A } & 105-120\end{array}$

BSR (top of highreflectivity zone) IR associated core water chemistry $\left(\mathrm{Cl}^{-}\right)$ anomaly

\section{IR associated core} water chemistry $\left(\mathrm{Cl}^{-}\right)$ anomaly

Core (pressure core) gas volume

Depleted $\mathrm{Cl}^{-}$concentrations with depth to the bottom of the hole

\section{Clay with limited silt/ sand beds} Possible pore- Microbial

Depleted $\mathrm{Cl}^{-}$concentrations

Depleted $\mathrm{Cl}^{-}$concentrations above BSR

$0.5-2.4 \% \mathrm{Sh}$

Thinly bedded, slightly elevated resistivity (0.5-1.0 $\Omega$-m) over background; Archie Sh ranging from 5 to $20 \%$ 
Table 5 (continued)

\begin{tabular}{|c|c|c|c|c|c|c|c|}
\hline Site & Hole & Depth (mbsf) & Indicator & Description & $\begin{array}{l}\text { Dominant } \\
\text { sediment type }\end{array}$ & $\begin{array}{l}\text { Gas hydrate } \\
\text { reservoir type }\end{array}$ & Gas source \\
\hline & A & $180-205$ & Well logs (resistivity) & $\begin{array}{l}\text { Thinly bedded, slightly elevated } \\
\text { resistivity }(0.5-1.0 \Omega \text {-m) over } \\
\text { background; Archie } \text { Sh }<20 \%\end{array}$ & & & \\
\hline \multicolumn{8}{|c|}{ Andaman Sea } \\
\hline \multirow[t]{6}{*}{$\mathrm{NGH}$} & & & & & $\begin{array}{l}\text { Clay/silt } \\
\text { (nannofossil } \\
\text { ooze) with } \\
\text { volcanic ash } \\
\text { beds }\end{array}$ & $\begin{array}{l}\text { Pore-filling } \\
\text { (ranging from } \\
\text { disseminated to } \\
\text { highly } \\
\text { saturated gas } \\
\text { hydrate in ash } \\
\text { beds) }\end{array}$ & $\begin{array}{l}\text { Microbial }+ \\
\text { Thermogenic }\end{array}$ \\
\hline & - & 608 & BSR (uncertain phase) & & & & \\
\hline & A & $250-608$ & $\begin{array}{l}\text { IR associated core } \\
\text { water chemistry }\left(\mathrm{Cl}^{-}\right) \\
\text {anomaly }\end{array}$ & $\begin{array}{l}\text { Depleted } \mathrm{Cl}^{-} \text {concentrations } \\
\text { associated with ash beds }\end{array}$ & & & \\
\hline & A & $328.0,412.7$ & $\begin{array}{l}\text { Core (pressure core) gas } \\
\text { volume }\end{array}$ & $0.1-0.6 \% \mathrm{Sh}$ & & & \\
\hline & A & 586.3 & $\begin{array}{l}\text { Core (pressure core) gas } \\
\text { volume }\end{array}$ & $7.6 \% \mathrm{Sh}$ & & & \\
\hline & B & $250-608$ & Well logs (resistivity) & $\begin{array}{l}\text { Slightly elevated resistivity ( } 0.5 \\
-2.0 \Omega-\mathrm{m} \text { ) over background; } \\
\text { Archie Sh ranging from } 5 \text { to } 40 \%\end{array}$ & & & \\
\hline \multicolumn{8}{|c|}{ Kerala-Konkan Basin } \\
\hline \multirow[t]{3}{*}{$\mathrm{NGH}$} & & & & & $\begin{array}{l}\text { Carbonate-rich } \\
\text { pelagic } \\
\text { sediments }\end{array}$ & $\begin{array}{l}\text { No evidence of } \\
\text { gas hydrate }\end{array}$ & $\begin{array}{l}\text { Below } \\
\text { detection }\end{array}$ \\
\hline & - & 217 & BSR (uncertain phase) & $\begin{array}{l}\text { Result of subtle changes in } \\
\text { formation density associated } \\
\text { with changes in lithology (not } \\
\text { related to the occurrence of gas } \\
\text { hydrate) }\end{array}$ & & & \\
\hline & A & - & $\begin{array}{l}\text { No log or core evidence } \\
\text { of gas hydrate }\end{array}$ & & & & \\
\hline
\end{tabular}

\section{Gas hydrate indicators}

Seismic: Bottom simulator reflectors (BSR).

Seismic: Direct indicators ("peak" leading high amplitude reflectors).

Core: Direct observations.

Core: Temperature from inferred scanning (IR).

Core: Interstitial water chemistry $\left(\mathrm{Cl}^{-}\right)$.

Core: Gas volume from pressure cores.

Well logs: Elevated resistivities and acoustic velocities.

Gas hydrate reservoir type

Pore-filling or grain-displacement.

Fracture-filling.

Dominant sediment type

Clay-silt-sand (ash).

$\mathrm{Sh}=$ Gas hydrate saturation.

mbsf $=$ meters below seafloor

a Archie analyses are known to overestimate gas hydrate saturations in fracture dominated reservoirs (Collett and Lee, 2012).

skate guided by a rail/belt system. This system automatically stitches images and produces a single IR image and temperature array for each core. The use of the IR core scanning system during NGHP-01 allowed for the rapid identification of gas hydrate for sampling from temperature anomalies on the surface of the core liner.

As summarized in Table 5 and described in the Kumar et al. (this issue), Krishna-Godavari and Mahanadi Basins, and Andaman Sea summary report, gas hydrate-bearing sediments within the recovered conventional cores were identified indirectly by cold IR anomalies before core sectioning and sub sampling. The core IR images for each of the sites cored during NGHP-01 are shown along with the core-derived interstitial water chlorinity analyses and pressure core-derived gas volumes in the basin summary report (Kumar et al., this issue). Because of the operational linkage of using IR imaging to guide the interstitial water sampling program, there is a strong connection between these two data sets as reviewed below in this report. The core IR images from seven of the eight sites cored in the Krishna-Godavari Basin exhibited anomalous cold core sections that were attributed to the dissociation of gas hydrates and were usually sampled for the analysis interstitial water chemistry (as discussed below). The IR core images from both sites cored in the Mahanadi Basin and the one site cored in the Andaman Sea also revealed anomalous cold core sections that were targeted for subsampling and interstitial water analysis.

2.4.6.3. Interstitial water chlorinity analysis. During most marine gas hydrate coring programs, interstitial water samples are obtained from cores to identify and quantify the occurrence of gas hydrate in recovered cores as one of the goals of the core geochemistry sampling program. In the case of NGHP-01, cold spots in the core IR images were interpreted to represent dissociating or dissociated gas hydrate, which were used to select interstitial water, headspace gas, void gas, and other samples. The selected thermally-anomalous core samples and additional selected background core samples (i.e., core sections with no thermal anomaly or evidence of gas hydrate) were taken to the chemistry lab on the ship for processing and analysis. 
The dissociation of gas hydrate during core recovery releases methane and fresh water, thus pore water that is fresher than the assumed background salinity or chlorinity level is commonly taken as an indication of the presence of gas hydrate. This process has been widely used to estimate gas hydrate concentrations (Kastner et al., 1995; Paull et al., 1996). For NGHP-01, a smoothed interstitial water chlorinity reference profile was determined from the general trend of measurements taken on background samples for each site to represent the non-hydrate-bearing portion of the cored section. Local low-chlorinity anomalies were used to identify intervals where gas hydrate has dissociated upon recovery and to provide estimates of the potential magnitude of in situ gas hydrate saturation.

Interstitial water chlorinity anomalies for the sites cored during NGHP-01 are noted in Table 5 and described in detail in Kumar et al. (this issue), which also provides the interstitial water chlorinity profiles, core IR images, and pressure core-derived gas volumes for each site cored during NGHP-01.

The integrated analysis of the IR core images and interstitial water chlorinity profiles show that the occurrence and concentration of gas hydrate varies considerably between sites cored in each basin during NGHP-01. All of the sites cored in the Krishna-Godavari Basin, the Mahanadi Basin, and the Andaman Sea exhibited interstitial water chemistry anomalies indicative of the presence of gas hydrate. The interstitial water chlorinity data from Site NGHP-01-1 in the Kerala-Konkan Basin does not reveal any evidence for the occurrence of gas hydrate (Kumar et al., this issue). Only Site NGHP-01-10 in the Krishna-Godavari Basin exhibited chloride concentrations exceeding that of seawater, with all of the other sites characterized by chloride concentrations and salinities lower than that of seawater.

The type of gas hydrate distribution in the Krishna-Godavari Basin (Kumar et al., this issue) has been subdivided into four groups based on the analysis of the interstitial water chlorinity profiles and core IR images (with additional input from the analysis of downhole well log data images, to be discussed later in this report) (Table 5): (1) sites with possible fracture-filling and/or pore-filling gas hydrate within discrete intervals with low pore space occupancy (Sites NGHP-01-03, -07, -14, -16 and -20); (2) sites with thick intervals of fracture-filling and/or pore-filling gas hydrate at moderate concentrations (Site NGHP-01-05); (3) sites with pore-filling gas hydrate within discrete coarser-grained sediment intervals at high gas hydrate saturations (Site NGHP-01-15); and (4) sites that contain massive gas hydrate associated with high-angle fractures in clay-dominated sediments (Sites NGHP-01-10, -12, and -21). In the Krishna-Godavari Basin, the highest chlorinity inferred gas hydrate concentrations appear to be associated with fracture systems in clay-rich sediments and several coarser-grained stratigraphic units, with the chlorinity inferred pore volume occupancy on the order of $<1 \%$ to a maximum of $\sim 61 \%$ at the three massive gas hydrate related fracture dominated sites that are all closely associated with Site NGHP-01-10 (i.e., Sites NGHP-01-10, -12 and -21). As discussed above, Sites NGHP-01-18 and NGHP-10-19 in the Mahanadi Basin were established as paired sites to test the sedimentary section overlying an apparent channelized free-gas accumulation. The interstitial water chlorinity profiles and core IR images from these two sites (Table 5) indicate the presence of minor quantities of disseminated gas hydrate throughout the sediments above the seismic-imaged channelized free-gas accumulation. At Site NGHP01-17 in the Andaman Sea, the occurrence of gas hydrate is primarily associated with volcanic ash horizons, with the chlorinity inferred gas hydrate concentrations ranging from $<1 \%$ to a maximum of $\sim 76 \%$. Overall, the gas hydrate saturation estimates based on pressure core-derived gas concentrations (discussed below) and core-derived chloride concentrations are similar.
2.4.6.4. Pressure core analysis. On NGHP-01, pressure cores retrieved at in situ pressures were used to determine gas hydrate distribution and quantity using both non-destructive measurement of the physical properties of the cores at in situ pressures and destructive methane gas collection and mass balance measurements (Table 3) (Collett et al., 2008a). The techniques used were similar to those developed on IODP Expedition 311 (Riedel et al., 2006). After a pressure core was retrieved, initial non-destructive measurements, including gamma density and compressionalwave velocity, as well as X-ray images were used to characterize the nature and distribution of gas hydrate within the sediment. Most of the cores were then depressurized on the ship to determine the total methane composition and concentration in the sediments. In some instances the cores were stored at conditions suitable for gas hydrate stability for further analyses in laboratories on shore.

During NGHP Expedition 01, the bulk of pressurized sediments were available for onboard depressurization experiments. Controlled release of pressure from pressure cores through a manifold was used to estimate the in situ abundance of gas hydrate (or free gas for cores acquired outside the GHSZ) based on mass balance calculations, methane solubility, and gas hydrate stability considerations as reported in Collett et al. (2008a). For cores acquired from within the inferred GHSZ, all the recovered gas was assumed to be in the form of gas hydrate or as dissolved gas in pore water.

Thirty-seven of the 97 pressure cores attempted during NGHP01 were recovered at sufficient pressure to assess the total concentration of methane and hence the predicted amount of gas hydrate. The methane concentrations calculated from the NGHP01 recovered pressure cores are shown in a series of core data images in Kumar et al., (this issue). The pressure core-derived gas hydrate saturations listed in Table 5 are commonly less than $1 \%$. Notable exceptions include a saturation value of $16.1 \%$ for a gas hydrate-bearing sand-silt reservoir section at Site NGHP-01-15 and a value of $31.5 \%$ in the fractured-reservoir system at Site NGHP-0121.

The many gas hydrate-bearing sediment samples observed during NGHP-01, both visually in cores on the catwalk and from the $\mathrm{X}$-ray images of pressure cores, provide insight into gas hydrate morphology and distribution in sediments. The catwalk visual observations from APC and XCB cores are limited to the more massive forms of gas hydrate, as the finer structures will have dissociated. However, the preserved morphologies in the pressure cores provided a unique opportunity to examine the in situ nature of gas hydrate from the non-destructive measurements (compressionalwave velocity and gamma density) as well as from the X-ray images.

Figure 7A, B shows the complete X-ray images of two wellpreserved FPC pressure cores from Hole NGHP-01-10B (Cores NGHP-01-10B-08Y and NGHP-01-10B-18Y) along with enlarged sections to illustrate the different gas hydrate morphologies observed. Both cores were retrieved near full in situ pressure. Core NGHP-01-10B-18Y X-ray image is characterized by greater lithological variability, with regions of dark and light intensity with sharp boundaries; whereas Core NGHP-01-10B-08Y shows only gradual changes in overall image intensity. In the more homogenous core (Core NGHP-01-10B-08Y) the gas hydrate takes the form of nodules (Fig. 7A - insert A), distinct horizontal veins (Fig. 7A insert $C$ ), and many thin wispy vertical/subvertical hydrate structures (Fig. 7A - inserts A, B, and C). In Core NGHP-01-10B-18Y, there are more distinct lithological changes and the only visible gas hydrate occurrence in horizontal layers (Fig. 7B), no vertical veins of gas hydrate are visible in this core.

Calculations show that only a small amount of the gas hydrate in Core NGHP-01-10B-18Y (299 mL) can be accounted for by the thin 

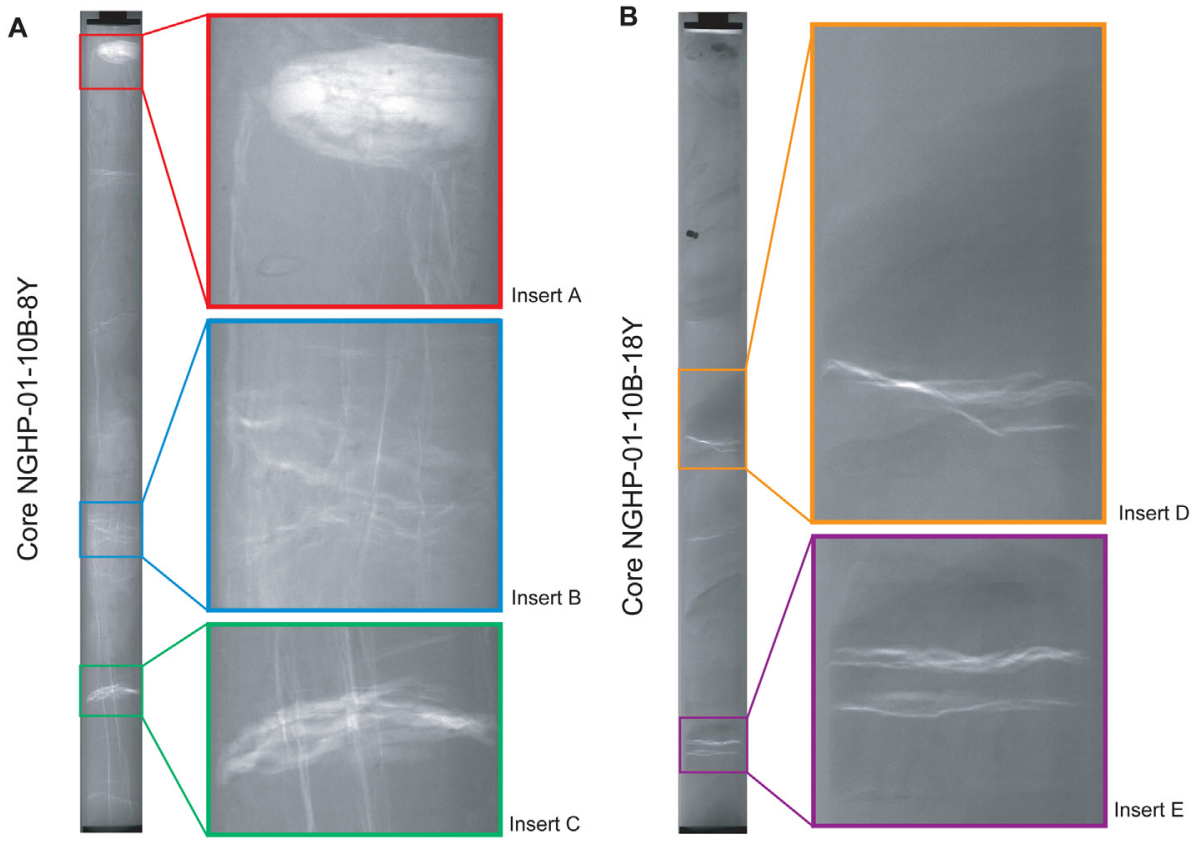

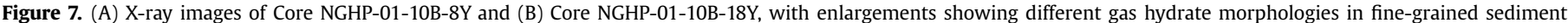
(modified from Collett et al., 2008a).

wispy mostly horizontal layers of gas hydrate that are visible on the X-ray (Collett et al., 2008a). A single layer $0.5 \mathrm{~cm}$ thick would only contain $13 \mathrm{~mL}$ of gas from gas hydrate. The bulk of the gas hydrate in this core must be in a more distributed form that is below the resolution of the X-ray images. This distributed gas hydrate could take two quite different forms:

1. Disseminated gas hydrate that replaces pore water, but does not substantially displace the sediment grain matrix. In this case, no substantial reduction in overall bulk density would occur and no change would be visible in the X-ray image. However, some increase in matrix rigidity could occur, resulting in increased $\mathrm{P}$ wave velocities.

2. Finely distributed grain-displacing gas hydrate, where fine grains of gas hydrate displace the sediment matrix and lower the overall density of the sediment, which should be visible in the gamma density data and X-ray image. Finely distributed grain-displacing gas hydrate may also have a "cementing" effect which could cause an increase in P-wave velocity.

It can be concluded that the low-density zones with diffuse boundaries in Core NGHP-01-10B-18Y, seen in the X-ray images in Figure $7 \mathrm{~B}$, contain some form of grain-displacing gas hydrate. The darker layers in the X-ray images of Core NGHP-01-10B-18Y correlated with local maxima in P-wave velocities, which further supports the interpretation that the intervals may have contained disseminated or grain-displacing gas hydrate in some form. However, X-ray images of Core NGHP-01-10B-08Y (Fig. 7A) and X-ray computed tomography scans of pressure cores performed postcruise (see Collett et al., 2008a) showed that all cores examined from Site NGHP-01-10 and nearby Site NGHP-01-21 contained pervasive, millimeter-scale veins of gas hydrate that were not visible on the original X-rays. These thin veins sometimes showed preferred orientations, which could affect the density and velocity profiles of the cores. Further studies of the pressure cores acquired during NGHP-01 (Dai and Santamarina, this issue), however, have suggested that the X-ray imaged vertical vein features seen in many of the NGHP-01 pressure cores may be a product of gas hydrate dissociation due to mechanical extension, negative pore pressure generation during unloading (Mandel-Cryer effect), and secondary hydrate formation in the form of the observed vertical vein features. The natural and/or core handling factors that may control the occurrence of the X-ray-imaged hydrate-filled vertical vein-like features is uncertain at this time.

The pressure cores acquired during NGHP-01 also provide additional insight into the occurrence of gas hydrate in silt- and sand-dominated reservoir systems. As summarized in Table 5 and documented in the drill site reviews in Kumar et al., (this issue), gas hydrates have been shown to occur in a limited number of sand reservoir systems in the offshore of India. One of the better developed, but relatively thin, hydrate-bearing sand reservoirs was discovered at Site NGHP-01-15 in the Krishna-Godavari Basin (Collett et al., 2008a; Riedel et al., 2011). Pressure Core NGHP-01$15 \mathrm{~A}-11 \mathrm{Y}$ and conventional Core NGHP-01-15A-09X reflect the range of gas hydrate occurrence at Site NGHP-01-15. Core NGHP01-15A-09X was associated with a distinct thermal anomaly and low pore-water chlorinity, which was acquired just above the NGHP-01-15A-11Y pressure core. Both cores were shown to contain gas hydrate in distinct sand layers, but many other sand layers in this hole did not appear to contain gas hydrate. Two sand layers within Core NGHP-01-15A-11Y (pressure core) (Fig. 8) were examined for the presence of gas hydrate, though the two layers were less than $50 \mathrm{~cm}$ apart. One showed a gas hydrate saturation of $15-20 \%$ of pore space and the other showed no gas hydrate presence at all, based on chlorinity measurements and methane mass balance calculations as described in Collett et al. (2008a). The mechanism by which gas hydrate was distributed between these closely spaced layers is not well understood. One-dimensional, vertical models of gas hydrate formation cannot explain such distributions of gas hydrate. More complex lateral transport and geologic models must be considered when examining the occurrence of gas hydrate at various scales.

2.4.6.5. Well $\log$ analysis. Advanced wireline and LWD tools are routinely used to examine the petrophysical properties of gas hydrate reservoirs and the distribution and concentration of gas 
Core NGHP-01-15A-11Y Depressurization

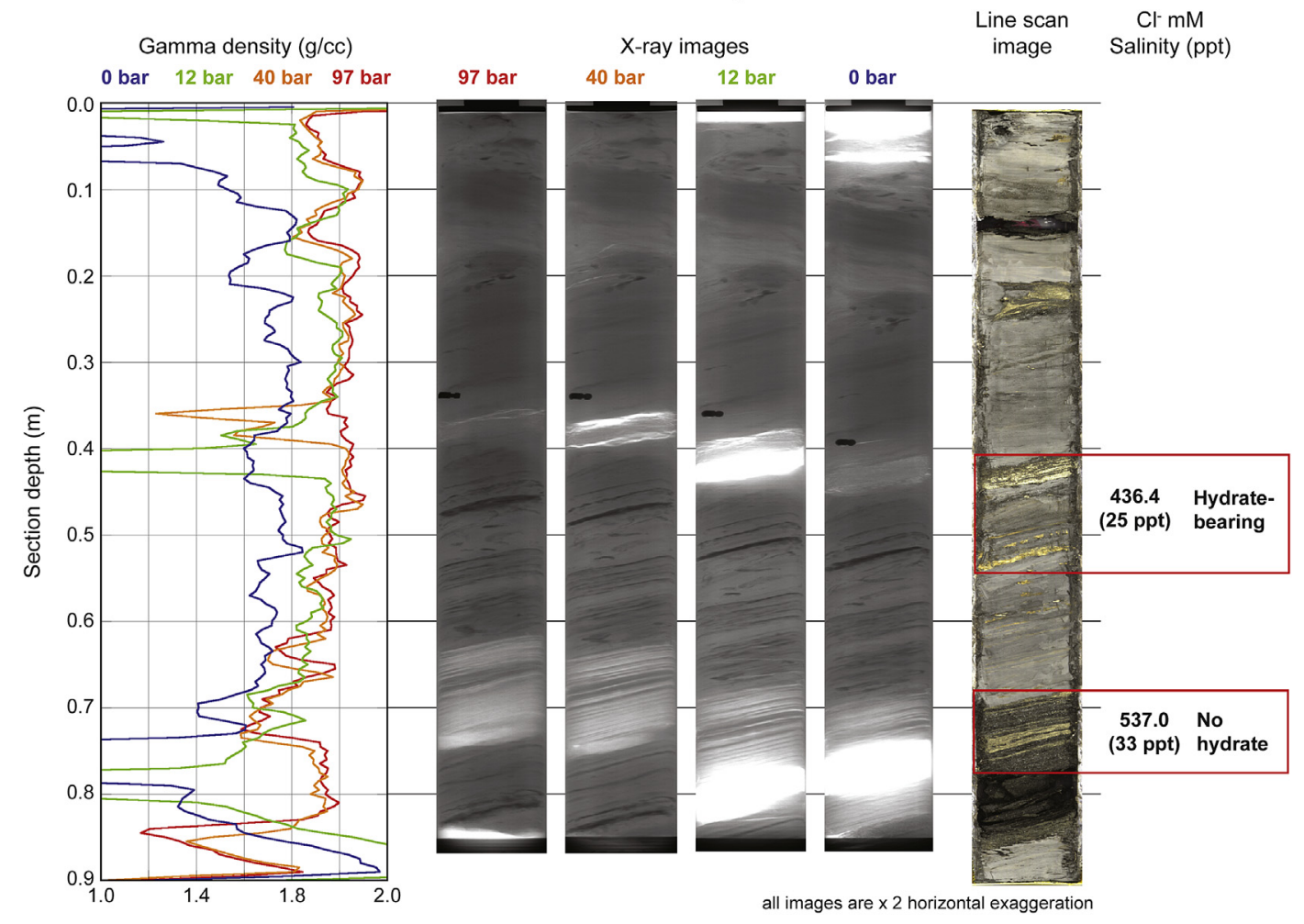

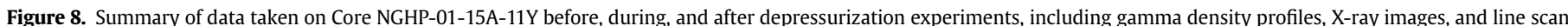

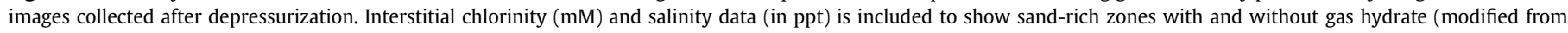
Collett et al., 2008a).

hydrates within various reservoir systems. The most established and well-known use of well logs in gas hydrate research are those that provide electrical resistivity and acoustic velocity data (both compressional- and shear-wave data) to estimate gas hydrate content (i.e., gas hydrate pore-volume saturation) in various types of reservoirs (Collett and Lee, 2012). Recent integrated sediment coring and well log studies have confirmed that electrical resistivity and acoustic velocity data can yield accurate gas hydrate saturations in sediment grain-supported (isotropic) systems such as sand reservoirs, but more advanced log-analysis models are required to characterize gas hydrate in fractured (anisotropic) reservoir systems (Lee and Collett, 2008, 2009; Cook et al., 2010). New well logging tools designed to make directionally oriented acoustic and propagation resistivity log measurements provide the data needed to analyze the acoustic and electrical anisotropic properties of both highly interbedded and fracture-dominated gas hydrate reservoirs.

The downhole logging program during NGHP-01 was specifically designed to assess the presence and concentration of gas hydrates. NGHP-01 acquired well logs have provided robust information about complex gas hydrate reservoir systems containing both pore- and fracture-filling gas hydrate (Collett et al., 2008b; Lee and Collett, 2008, 2009; Cook et al., 2010; Shankar and Riedel, 2011; this issue). As discussed above and noted in Table 2, 12 LWD holes were drilled at 10 of the sites established during Leg 2 of NGHP-01. An additional 11 holes were wireline logged during NGHP-01, including 1 in the Kerala-Konkan Basin, 8 in the Krishna-Godavari Basin, 1 in the Mahanadi Basin, and 1 in the Andaman Sea.

Well log displays for each of LWD holes drilled in the Krishna-Godavari (Sites NGHP-01-2, -03, -04, -05, -06, -07, -10, and -11) and the Mahanadi (Sites NGHP-01-08 and -09) Basins, as well as displays of the wireline log data acquired from holes in the Krishna-Godavari Basin (Sites NGHP-01-14, -15, and -16), the Mahanadi Basin (Site NGHP-01-19), the Andaman Sea (Site NGHP-01-17), and the Kerala-Konkan Basin (Site NGHP-01-1), are included in Kumar et al., (this issue). Each of the LWD and wireline log displays include gamma-ray, formation density, neutron and density-derived porosity, and resistivity log data traces. In the case of the LWD holes, each log display also includes resistivity-at-bit (RAB) electrical resistivity image of the borehole as obtained by the geoVISION tool. Each LWD and wireline log display also includes plots of the Archie-derived (Archie, 1942; Collett et al., 2008a) gas hydrate saturations (the percent of pore space occupied by gas hydrate) assuming isotropic reservoir conditions.

All eight of the LWD sites established during Leg 2 of NGHP-01 in the Krishna-Godavari Basin exhibited a range of resistivity log values, some of which are greater than the background measurements, which may be indicative of the presence of hydrocarbons. When such resistivity anomalies are noted within the inferred GHSZ and are associated with sediment with background or greater log-derived porosity values, gas hydrate occurrence can be inferred (Table 5) (Kumar et al., this issue). The LWD resistivity log data and associated Archie-derived gas hydrate saturations from Sites NGHP-01-02, and -03 do not reveal any evidence of significant occurrence of gas hydrates other than several possible fracturefilling and/or pore-filling gas hydrate occurrences within discrete intervals with gas hydrate saturations generally $<20 \%$. The LWD resistivity log data from Sites NGHP-01-04, -05, -06, -07, and -11 in the Krishna-Godavari Basin are characterized by thick intervals with elevated resistivities (ranging from 0.5 to $5.0 \Omega-\mathrm{m}$ ) that are interpreted to be fracture-filling and/or pore-filling gas hydrate- 
bearing sections at moderate saturations (ranging as high as 30-50\%). ${ }^{1}$ The wireline-acquired log data from Sites NGHP-01-14 and -16 in the Krishna-Godavari Basin also exhibit distinct intervals with elevated resistivities (ranging from 0.5 to $5.0 \Omega-\mathrm{m}$ ) that are interpreted to be fracture-filling and/or pore-filling gas hydrate-bearing sections at moderate saturations (ranging as high as $25-50 \%)^{1}$ All saturation estimates presented in this report use the Archie formulation assuming isotropic hydrocarbon distribution.

Of the four holes drilled at Site NGHP-01-10 in the Krishna-Godavari Basin, LWD data were collected in Hole NGHP-01-10A, and Hole NGHP-01-10D was wireline logged (Kumar et al., this issue). Gas hydrate was inferred from the LWD data at Site NGHP01-10 within the depth interval from $\sim 25$ to $\sim 160 \mathrm{mbsf}$. The analysis of RAB borehole electrical resistivity images and the pressure core X-ray scans confirm the fracture-filling nature of the gas hydrate occurrence at Site NGHP-01-10 (Riedel et al., 2010). The standard Archie's resistivity log-derived gas hydrate saturations are about $85 \%$ in the interval $27-90$ mbsf $^{1}$ and decrease slightly to $50-75 \%$ in the interval $90-160$ mbsf. $^{1}$ But it is critical to note that resistivity-derived gas hydrate saturations in fracture-dominated reservoirs are often higher than that obtained from pressure-core degassing (Hadley et al., 2008), which are considered the most reliable in situ saturation measurement. These concerns are dealt with in greater detail in Lee and Collett (2009) and later in this report.

One of the more distinct well log and core inferred pore-filling gas hydrate within a discrete coarser-grained sedimentary section was found in the Krishna-Godavari Basin at Site NGHP-01-15 (Riedel et al., 2010). The relatively high wireline log-measured resistivities (ranging from 0.5 to $5.0 \Omega-\mathrm{m}$ ) throughout most of the stratigraphic section above the projected depth of the BSR (126 mbsf) suggests that some gas hydrate is present throughout most of the logged interval. The zone with the highest resistivity values at depth of 75-81 mbsf correspond to the section inferred to be gas hydrate-bearing based on the presence of a prominent core IR temperature anomaly and diluted interstitial pore-water chlorinities. Archie-derived gas hydrate saturations reach a maximum around $50 \%$ near a depth of $80 \mathrm{mbsf}$.

The resistivity log data from the two Mahanadi Basin LWD drilled sites (Site NGHP-01-08 and -09) yielded only limited evidence of gas hydrates, with resistivity values elevated only $\sim 0.2 \Omega$ $\mathrm{m}$ over background equating to an Archie-derived gas hydrate saturations of $<10 \%$ (Table 5) (Kumar et al., this issue; Shankar and Riedel, this issue). The wireline log data from Site NGHP-01-19, however, reveals a distinct trend of increasing resistivity log values with depth toward the BGHSZ and the underlying seismicinferred channelized free-gas accumulation.

Gas hydrate within the ash beds and ash-rich stratigraphic units at Site NGHP-01-17 in the Andaman Sea, as inferred from numerous core IR and pore-water $\mathrm{Cl}^{-}$anomalies in the depth interval from $\sim 250$ mbsf to the BSR at 608 mbsf, also exhibit slightly elevated resistivity log values (ranging from 0.5 to $2.0 \Omega$-m over background) on the wireline log data acquired in Hole NGHP-01-17B (Table 5) (Kumar et al., this issue). The Archie-derived gas hydrate saturations are as much as $40 \%$ in several of the thicker ash-rich units near the depth of the BSR at Site NGHP-01-17.

The wireline log data from Site NGHP-01-1 in the Kerala-Konkan Basin did not provide any evidence for the presence of gas hydrate (Table 5) (Kumar et al., this issue).

\footnotetext{
${ }^{1}$ Archie analyses are known to overestimate gas hydrate saturations in fracturedominated reservoirs (Collett and Lee, 2012).
}

As noted above and reviewed in Lee and Collett $(2008,2009)$ and Cook et al. (2010, this issue), RAB borehole electrical resistivity images and pressure core X-ray scans can be used to effectively differentiate pore-filling gas hydrate occurrences from fracture-filling gas hydrates. For the most part, resistivity logderived gas hydrate saturations (using traditional Archie calculations that assume isotropic reservoir conditions) in the sandrich isotropic reservoirs compare favorably with gas hydrate saturations derived from pressure core gas volumetric analysis and core-derived interstitial water chlorinity freshening. However, resistivity-derived gas hydrate saturations vary greatly from core-derived gas hydrate saturations in the fracturedominated gas hydrate reservoirs (Hadley et al., 2008; Collett and Lee, 2012).

Pressure cores from Site NGHP-01-10, as described above, shows thatthe occurrence of gas hydrate at this site ranges from a disseminated or grain-displacing form to a pervasive small-scale fracture- and vein-fill material. But at a larger scale, Lee and Collett (2008) and Riedel et al. (2010) have shown that the prominent gas hydrate occurrence at Site NGHP-01-10 in the Krishna-Godavari Basin is primarily controlled by the presence of a larger scale through-going near-vertical fracture system. Assuming the resistivity of gas hydrate-bearing sediments is isotropic, the conventional Archie analysis using the LWD resistivity log from this site yields gas hydrate saturations greater than $50 \%$ (as high as $85 \%$ ) of the pore space for the depth interval between 27 and 160 mbsf. However, gas hydrate saturations estimated from pressure cores from nearby wells were less than $26 \%$ of the pore space. The primary cause of the saturation difference is attributed to the anisotropic nature of the reservoir due to gas hydrate in high-angle fractures. Lee and Collett (2008) showed that the Archie relationship can be used to estimate gas hydrate saturations in an anisotropic reservoir, with additional information such as elastic velocities to constrain various Archie parameters, which compare more favorably with gas hydrate saturations derived from other sources. This type of advanced analysis for every well logged during NGHP-01 is beyond the scope of this summary or the associated basin summary report. However, it is noted in Table 5 and each of the associated well log displays in the basin summary report the Archie-derived gas hydrate saturations that may have been impacted by the anisotropic reservoir properties associated with gas hydrate-filled vertical fractures.

2.4.6.6. Nature of gas hydrate occurrence - summary. As previously described in this report, summarized in Table 5, and reviewed in Kumar et al., (this issue), gas hydrate at the drill sites established during NGHP-01 occurred mostly as fracture-filling material associated with large-scale fault systems or within local, smallscale fractures often of limited size. Gas hydrate also occurred as pore-filling in mostly fine-grained sediment within zones of variable thickness. There were only a limited number of discrete coarser-grained sediment units (i.e., coarse silts, fine sands, and ash beds) that exhibited relatively high gas hydrate saturations. In the case of gas hydrate-bearing sandy- and ash-rich sediments, cold IR anomalies were only found within the coarse-grained portions of the recovered cores. This type of pore-filling gas hydrate shows a direct proportional increase in the gas hydrate saturation with increases in sediment grain size, which is consistent with previous findings from IODP Expedition 311 (Torres et al., 2008) and in the Ulleung Basin offshore Korea (Bahk et al., 2013).

Gas hydrates in the Krishna-Godavari Basin occur over a wide range of conditions, from highly disseminated or distributed occurrences at low saturations over thick intervals of the wells drilled 
in the basin to distinct isolated fracture-filling and strata-bound pore-filling gas hydrate occurrences (Kumar et al., this issue). The massive gas hydrate occurrences associated with high-angle fractures in the clay-dominated sediments at Site NGHP-01-10 (and associated Sites NGHP-01-12 and -21) was a unique discovery and appears to be the result of a specific combination of faulting and gas migration from a deeper gas source (Riedel et al., 2010). However, there are similar features throughout the basin. Additional detailed analysis of $\mathrm{RAB}$ electrical resistivity boreholes images in the Krishna-Godavari Basin (Cook and Goldberg, 2008) also reveal the presence of abundant gas hydrate-filled fractures of limited extent, with the imaged fractures extending only a few meters. Because these types of gas hydrate filled fractures appear to be unconnected to the base of the GHSZ, it was suggested by Cook and Goldberg (2008) that these small planar fractures may be generated by gas formed locally by microbes.

The occurrence of gas hydrate in the Mahanadi Basin appears to be mostly controlled by the lithostratigraphic history of the basin, which is characterized by numerous large canyon cut-and-fill features along the upper slope-rise portion of the basin as drilled during NGHP-01 (Kumar et al., this issue). The pre-drill analysis of seismic data from the basin revealed what appeared to be highamplitude gas charged cut-and-fill canyon features, the top of which correlated to the base of the expected GHSZ. Analysis of recovered core and well log data showed that the high-amplitude portion of the canyon cut-and-fill features contained mostly finegrained sediments with relatively low free-gas saturations $(<20 \%)$ and the canyon cut-and-fill features that extended into the overlying methane hydrate stability zone also contained mostly finegrained sediments with relatively low gas hydrate saturations $(<20 \%)$.

Gas hydrate was also discovered at the only NGHP-01 site established along the convergent margin setting in the Andaman Sea (Kumar et al., this issue). Well log and core data from Site NGHP-01-17 revealed the occurrence of pore-filling gas hydrate in volcanic ash beds and ash-rich zones (Shankar and Riedel, 2011). In this unique geologic setting, the relatively coarse-grained nature of the volcanic ash provided connected pore space in which gas hydrate could be concentrated with peak saturations exceeding $50 \%$ of the available pore space.

As previously reviewed in this report, neither the well log nor the core data from Site NGHP-01-1 in the Kerala-Konkan Basin, along the west coast of India, exhibited any evidence for the presence of gas hydrate (Table 5) (Kumar et al., this issue).

\section{Future research and direction}

The occurrence of gas hydrate in mostly fracture-dominated systems in the offshore of India as documented during NGHP-01 is somewhat troubling when considering their energy resource potential. The prospects for economic recovery of natural gas from fracture-filling forms of gas hydrate occurring in mostly finegrained sediments is likely limited with current technologies, and major improvements in extraction methods are required to enable commercial exploitation of such deposits (as reviewed by Collett et al., 2009; Boswell et al., 2014).

It is well established that intrinsic reservoir permeability, relative permeability, fluid distribution, porosity, and hydrocarbon saturation typically control fluid flow in conventional gas reservoirs. Gas hydrate adds complexity to the controls on flow in the reservoir. For gas to flow from the reservoir into a producing well, it first has to be released from the hydrate structure. Laboratory and modeling studies along with field tests have shown that depressurization within a permeable reservoir may be the most promising technique for the production of gas hydrates. One of the key factors expected to control the efficiency of hydrate dissociation by depressurization and gas flow to the well include the intrinsic and relative permeability of the hydrate-bearing reservoirs. It has been shown that initial intrinsic and post production permeability of fine-grained fracture-dominated gas hydrate occurrences are very low (as reviewed by Moridis et al., 2008); thus, limiting the effectiveness of depressurization on production of the gas hydrate from these types of systems.

Although detailed information on the production characteristics of gas hydrate is rather limited, there is enough information to assume that the more promising gas hydrate production targets (as reviewed by Moridis et al., 2008) are those in high-quality (high porosities and intrinsic permeabilities) sand-rich reservoirs with high gas hydrate saturations. It is anticipated that future NGHP efforts will likely include drilling, coring, and field production testing. Recent NGHP research activities have included the addition of a new site review effort, which has focused on the potential for sand-rich drilling targets and gas hydrate prospects in deeper water. It has been proposed that in 2015 or possibly 2016, NGHP Expedition 02 may be constituted to drill, log, and core several promising gas hydrate prospects in the offshore of India.

\section{Summary}

NGHP-01 was among the most complex and comprehensive gas hydrates field ventures yet conducted. The successful recovery of a wealth of log and core data over a range of geologic environments has provided invaluable information on the geologic controls on the occurrence of gas hydrate in nature. The NGHP-01 expedition was an excellent example of the international spirit of collaboration that continues to infuse gas hydrates research.

The operational highlights of NGHP-01 included the following:

- 113.5 days of operation without any reportable injury or incident.

- Only $1 \%$ of total operation time was down time due to equipment malfunction or weather.

- Examination of 9,250 m of sedimentary section at 39 locations within 21 sites located in four geologically distinct settings.

- Collected LWD log data in 12 holes at 10 sites.

- Collected wireline log data at 13 sites.

- Collected vertical seismic profile data at six sites.

- Collected 494 conventional cores, encompassing about $2,850 \mathrm{~m}$ of sediment, from 21 holes (79\% overall recovery).

- Collected detailed, shallow geochemical profiles at 13 sites.

- Established temperature gradients at 11 sites.

- Extensive sample collection to support a wide range of postcruise analyses, including:

- Collected about 6800 whole-round core samples for examination of interstitial water geochemistry, microbiology, and other analyses.

- Collected more than 12,500 core subsamples for paleomagnetic, mineralogical, paleontological, and other analyses.

- Collected about 140 gas hydrate-bearing sediment samples for storage in liquid nitrogen.

- Conducted 97 deployments of advanced pressure coring devices, resulting in the collection of 49 cores that contain virtually undisturbed gas hydrate in host sediments at near in situ pressures.

- Collected five 1-m-long gas hydrate-bearing pressure cores for analysis of the physical and mechanical properties of gas hydrate-bearing sediment.

- Collected 21 re-pressurized cores (nine representing subsamples from gas hydrate-bearing pressure cores). 
The key scientific highlights of NGHP-01 included the following:

- Conducted comprehensive analyses of gas hydrate-bearing marine sediments in passive continental margins and marine accretionary wedge settings.

- The calculated depth to the base of the methane hydrate stability zone, as derived from downhole temperature measurements, closely matches the calculated depth of the seismically identified bottom simulating reflector (BSR) at most of the sites established during this expedition.

- Discovered gas hydrate in numerous complex geologic settings and collected an unprecedented number of gas hydrate-bearing cores.

- Most of the recovered gas hydrate was characterized as a fracture-filling material in fine-grained (mostly clay-rich) sediments. A lesser volume of gas hydrate was found as pore-filling particles disseminated in coarser-grain sediments.

- The occurrence of concentrated gas hydrate is mostly controlled by the presence of fractures and/or permeable, coarser-grained sediments.

- Gas hydrate was found occurring in "combination reservoirs" consisting of horizontal or subhorizontal coarse-grained permeable sediments (silts for the most part) and apparent vertical to subvertical fractures that provide the conduits for gas migration.

- Delineated and sampled one of the thickest marine gas hydrate accumulations yet discovered (Site NGHP-01-10 in the Krishna-Godavari Basin).

- Discovered one of the deepest gas hydrate occurrences known at the time (offshore of the Andaman Islands, Site NGHP-01-17) which revealed gas hydrate-bearing volcanic ash layers as deep as $600 \mathrm{~m}$ below the seafloor.

- Established the existence of a fully developed gas hydrate system in the Mahanadi and the Krishna-Godavari Basins in the Bay of Bengal.

- Most of the gas hydrate occurrences discovered during this expedition contain mostly methane which was generated by microbial processes. However, there is also evidence of a thermal origin for a portion of the gas within the hydrates in the Andaman Sea offshore area.

- In at least one case, gas hydrate in the Krishna-Godavari Basin appears to be closely associated with large-scale structural features, in which the flux of gas through local fracture systems, generated by the regional stress regime, controls the occurrence of gas hydrate.

\section{Acknowledgment}

The editors wish to thank those that contributed to the success of the Indian National Gas Hydrate Program Expedition 01 (NGHP01). NGHP-01 was planned and managed through collaboration between the Directorate General of Hydrocarbons (DGH) under the Ministry of Petroleum and Natural Gas (India), the U.S. Geological Survey (USGS), and the Consortium for Scientific Methane Hydrate Investigations (CSMHI) led by Overseas Drilling Limited (ODL) and FUGRO McClelland Marine Geosciences (FUGRO). The platform for the drilling operation was the research $D / V$ JOIDES Resolution, operated by ODL. Much of the drilling/coring equipment used was provided by the Integrated Ocean Drilling Program (IODP) through a loan agreement with the U.S. National Science Foundation. Wireline pressure coring systems and supporting laboratories were provided by IODP/Texas A\&M University (TAMU), FUGRO, USGS, U.S. Department of Energy (USDOE) and HYACINTH/GeoTek. Downhole logging operational and technical support was provided by Lamont-Doherty Earth Observatory (LDEO) of Columbia University. The financial support for the NGHP-01, from the Oil
Industry Development Board, Oil and Natural Gas Corporation, GAIL, and OIL India is gratefully acknowledged. We also acknowledge the support extended by all the participating organizations of the NGHP: MOP\&NG, DGH, ONGC, GAIL, OIL, NIO, NIOT, and RIL. The authors and editors are thankful to the Management of the National Gas Hydrate Program, DGH and ONGC for permission to publish this report in the Journal of Marine and Petroleum Geology.

This report was funded in part by the U.S. Geological Survey Energy Resources Program and the U.S. Department of Energy under Interagency Agreement No. DE-AI21-92MC29214. Any use of trade, product, or firm names is for descriptive purposes only and does not imply endorsement by the U.S. Government.

\section{References}

Archie, G.E., 1942. The electrical resistivity $\log$ as an aid in determining some reservoir characteristic. J. Pet. Technol. 5, 1-8.

Ashford, D.I., Mizuta, T., Dallimore, S.R., Yamamoto, K., Nixon, F.M., Imasato, Y., Wright, J.F., Taylor, A.E., Applejohn, A., 2012. Overview of engineering and operations activities conducted as part of the JOGMEC/NRCan/Aurora Mallik 20072008 Gas hydrate production research well program, Part A: 2007 field program, in scientific results from the JOGMEC/NRCan/Aurora Mallik 2007-2008 Gas hydrate production research well program, Mackenzie Delta, northwest Territories, Canada. Geol. Surv. Can. Bull. 601, 25-51.

Bahk, J.-J., Kim, D.-H., Chun, J.-H., Son, B.-K., Kim, J.-H., Ryu, B.-J. Torres, M. Riedel, M., Schultheiss, P., 2013. Gas hydrate occurrences and their relation to host sediment properties: results from second Ulleung Basin gas hydrate drilling expedition, East Sea, Korea. J. Mar. Pet. Geol. 47, 21-29.

Boswell, R., Collett, T.S., 2011. Current perspectives on gas hydrate resources. Energy Environ. Sci. 4, 1206-1215.

Boswell, R., Collett, T.S., Dallimore, S., Frye, M., 2012b. Geohazards Associated with Naturally-occurring Gas Hydrate. In: Fire-In-The-Ice Methane Hydrate Newsletter, vol. 12, no. 1. National Energy Technology Laboratory, U.S. Department of Energy, pp. 11-15.

Boswell, R., Collett, T., Frye, M., Shedd, W., McConnell, D., Shelander, D., 2012a. Subsurface gas hydrates in the northern Gulf of Mexico. Mar. Pet. Geol. 34, 4-30.

Boswell, R., Yamamoto, K., Lee, S.-R., Collett, T.S., Kumar, P., Dallimore, S., 2014. Chapter 8, methane hydrates. In: Letcher, T.M. (Ed.), Future Energy. Elsevier Publishing, pp. 159-178.

Chandra, K., Singh, R.P., Julka, A.C., 1998. Gas hydrate potential of Indian offshore area. In: Proceedings of the 2nd Conference and Exposition on Petroleum Geophysics (SPG '98), January 19-21, 1998, Hotel Taj Coromandel, Chennai, India, $5 \mathrm{p}$.

Chopra, N.N., 1985. Gas hydrate an unconventional trap in forearc regions of the Andaman offshore. Bull. Oil Nat. Gas Corp. 22 (1), 41-54.

Collett, T., Boswell, R. (Eds.), 2012. Resource and Hazard Implications of Gas Hydrates in the Northern Gulf of Mexico: Results of the 2009 Joint Industry Project Leg II Drilling Expedition. Marine Pet. Geol. vol. 34, 1-209.

Collett, T.S., Boswell, R., Cochran, J.R., Kumar, P., Lall, M., Mazumdar, A. Ramana, M.V., Ramprasad, T., Riedel, M., Sain, K., Sathe, A.V., Vishwanath, K., the NGHP Expedition 01 Scientific Party, 2014. Geologic implications of gas hydrates in the offshore of India: results of the National Gas Hydrate Program Expedition 01. J. Mar. Pet. Geol. (in this issue).

Collett, T.S., Johnson, A., Knapp, C., Boswell, R., 2009. Natural Gas hydrates - a review. In: Collett, T., Johnson, A., Knapp, C., Boswell, R. (Eds.), Natural Gas Hydrates - Energy Resource Potential and Associated Geologic Hazards, American Association of Petroleum Geologists Memoir 89, 68 p.

Collett, T.S., Lee, M.W., October 2012. Well log characterization of natural gas hydrates. Petrophysics 53 (5), 348-367.

Collett, T., Riedel, M., Cochran, J., Boswell, R., Presley, J., Kumar, P., Sathe, A., Sethi, A. Lall, M., Siball, V., the NGHP Expedition 01 Scientific Party, 2008a. Indian National Gas Hydrate Program Expedition 01 Initial Reports. Prepared by the U.S. Geological Survey and Published by the Directorate General of Hydrocarbons. Ministry of Petroleum \& Natural Gas, India, 1 DVD.

Collett, T., Riedel, M., Cochran, J., Boswell, R., Presley, J., Kumar, P., Sathe, A., Sethi, A., Lall, M., Siball, V., Guerin, G., Malinverno, A., Mrozewski, S., Cook, A. Sarker, G., Broglia, C., Goldberg, D., the NGHP Expedition 01 Scientific Party, 2008b. Indian National Gas Hydrate Program Expedition 01 Downhole Log Data Report. Prepared by the U.S. Geological Survey and Published by the Directorate General of Hydrocarbons. Ministry of Petroleum \& Natural Gas, India, 2 DVD set.

Cook, A.E., Anderson, B.I., Malinverno, A., Mrozewski, S., Goldberg, D.S., 2010. Electrical anisotropy due to gas hydrate-filled fractures. Geophysics 75 (6), F173-F185.

Cook, A.E., Goldberg, D., 2008. Extent of gas hydrate-filled fracture planes: implications for in situ methanogenesis and resource potential. Geophys. Res. Lett. 35, L15302.

Cook, A.E., Goldberg, D.S., Malinverno, A., 2014. Natural gas hydrates occupying fractures: a focus on non-vent sites on the Indian continental margin and the northern Gulf of Mexico. J. Mar. Pet. Geol. (in this issue). 
Dai, S., Santamarina, J.C., 2014. Sampling natural hydrate-bearing sediments: pressure core technology used in the Krishna-Godavari Basin. J. Mar. Pet. Geol. (in this issue).

Directorate General of Hydrocarbons, 2014. Gas Hydrate: Road Map. Directorate General of Hydrocarbons, Noida, India, 2 p. http://www.dghindia.org/ NonConventionalEnergy.aspx?tab $=0$.

Hadley, C., Peters, D., Vaighan, A., 2008. Gumusut-Kakap Project: geohazard characterization and impact on field development plans. In: Proceedings of the International Petroleum Technology Conference, December 3-5, 2008, Kuala Lumpur, Malaysia, 15 p.

Holland, M., Schultheiss, P.J., 2014. Comparison of methane mass balance and X-ray computed tomography for calculation of gas hydrate content of pressure cores. J. Mar. Pet. Geol. (in this issue).

Inks, T.L., Lee, M.W., Agena, W.F., Taylor, D.J., Collett, T.S., Hunter, R.B., Zyrianova, M.V., 2009. Seismic prospecting for gas hydrate and associated freegas prospects in the Milne Point area of northern Alaska. In: Collett, T., Johnson, A., Knapp, C., Boswell, R. (Eds.), Natural Gas Hydrates - Energy Resource Potential and Associated Geologic Hazards, American Association of Petroleum Geologists Memoir 89, pp. 555-583.

Kastner, M., Kvenvolden, K.A., Whiticar, M.J., Camerlenghi, A., Lorenson, T.D., 1995. Relation between pore fluid chemistry and gas hydrate associated with bottomsimulating reflectors at the Cascadia Margin, Sites 889 and 892. In: Proceedings of the ODP, Scientific Results, 146 (Part 1). Ocean Drilling Program, College Station, TX, pp. 175-187.

Kumar, P., Collett, T.S., Boswell, R., Cochran, J.R., Lall, M., Mazumdar, A., Ramana, M.V., Ramprasad, T., Riedel, M., Sain, K., Sathe, A.V., Vishwanath, K., the NGHP Expedition 01 Scientific Party, 2014. Geologic implications of gas hydrates in the offshore of India: Krishna-Godavari Basin, Mahanadi Basin, Andaman Sea, Kerala-Konkan Basin. J. Mar. Pet. Geol. (in this issue).

Lee, M.W., Collett, T.S., 2008. Integrated analysis of well logs and seismic data at the Keathley Canyon, Gulf of Mexico, for estimation of gas hydrate concentrations. J. Mar. Pet. Geol. 25, 924-931.

Lee, M.W., Collett, T.S., 2009. Gas hydrate saturations estimated from fractured reservoir at site NGHP-01-10, Krishna-Godavari Basin, India. J. Geophys. Res. 114, 1-13. B07102.

Lee, M.W., Collett, T.S., Inks, T.L., 2009. Seismic attribute analysis for gashydrate and free-gas prospects on the North Slope of Alaska. In: Collett, T., Johnson, A. Knapp, C., Boswell, R. (Eds.), Natural Gas Hydrates - Energy Resource Potential and Associated Geologic Hazards, American Association of Petroleum Geologists Memoir 89, pp. 541-554.

McConnell, D., Zhang, Z., 2005. Using Acoustic Inversion to Image Buried Gas Hydrate Distribution. In: Fire-In-The-Ice Methane Hydrate Newsletter, vol. 5, no. 4. National Energy Technology Laboratory, U.S. Department of Energy, pp. 3-5.

Moridis, G.J., Collett, T.S., Boswell, R., Kurihara, M., Reagan, M.T., Sloan, E.D., Koh, C., 2008. Toward production from gas hydrates: assessment of resources and technology and the role of numerical simulation. In: Proceedings of the 2008 SPE Unconventional Reservoirs Conference, Keystone, Colorado, February, 10-12, 2008, SPE 114163, 45 p.

Paull, C.K., Matsumoto, R., Wallace, P.J. (Eds.), 1996. Initial Reports-Gas Hydrate Sampling on the Blake Ridge and Carolina Rise. Proceedings of the Ocean Drilling Program, Prepared by the Ocean Drilling Program, Texas A\&M University, College Station, Texas, vol. 164, 623 p.

Ramana, M.V., Ramprasad, T., Desa, M., Sathe, A.V., Sethi, A.K., 2006. Gas hydraterelated proxies inferred from multidisciplinary investigations in the Indian offshore areas. Curr. Sci. 91, 183-189.

Rao, Y.H., 1999. C- program for the calculation of gas hydrate stability zone thickness. Comput. Geosci. 25, 705-707.

Rao, Y.H., 2001. Gas Hydrate Investigations Along the Continental Margins of India (Ph.D. dissertation). Osmania University, Hyderabad, India, 234 p.

Rao, Y.H., Reddy, S.I., Khanna, Ramesh, Rao, T.G., Thakur, N.K., Subrahmanyam, C., 1998. Potential distribution of methane hydrates along the Indian continental margins. Curr. Sci. 74 (5), 466-468.

Rao, Y.H., Subrahmanyam, C., Rastogi, A., Deka, B., 2001a. Anomalous seismic reflections related to gas/gas hydrate occurrences along the western continental margin of India. Geo-Marine Lett. 21, 1-8.

Rao, Y.H., Subrahmanyam, C., Rastogi, A., Deka, B., 2002. Slope failures along the western continental margin of India: a consequence of gas-hydrate dissociation, rapid sedimentation rate, and seismic activity. Geo-Marine Lett. 22, 162-169.

Rao, Y.H., Subrahmanyam, C., Sharma, S.R., Rastogi, A.A., Deka, B., 2001b. Estimates of geothermal gradients and heat flow from BSRs along the Western Continental Margin of India. Geophys. Res. Lett. 28 (2), 355-358.
Rastogi, A., Deka, B., Budhiraja, I.L., Agarwal, G.C., 1999. Possibility of large deposits of gas hydrates in deeper water of India. Mar. Georesources Geotechnol. 17, 49-63.

Riedel, M., Collett, T.S., Kumar, P., Sathe, A.V., Cook, A., 2010. Seismic imaging of a fractured gas hydrate system in the Krishna-Godavari Basin offshore India. J. Mar. Pet. Geol. 27, 1476-1493.

Riedel, M., Collett, T.S., Malone, M.J., the Expedition 311 Scientists, 2006. Cascadia Margin Gas Hydrates, Expedition 311, Sites U1325-u1329, 28 August - 28 October, 2005, vol. 311. Integrated Ocean Drilling Program Management International, Inc., for the Integrated Ocean Drilling Program, p. 247. http://iodp. tamu.edu/publications/exp311/311 title.htm.

Riedel, M., Collett, T.S., Shankar, U., 2011. Documenting channel features associated with gas hydrates in the Krishna-Godavari Basin, offshore India. Mar. Geol. 279 $(1-4), 1-11$.

Ruppel, C.D., 2011. Methane hydrates and contemporary climate change. Nat. Educ Knowl. 3 (10), 29 p.

Saeki, T., Fujii, T., Inamori, T., Kobayashi, T., Hayashi, M., Nagakubo, S., Tokano, O. 2008. Extraction of Methane Hydrate Concentrated Zone for Resource Assessment in the Eastern Nankai Trough. Japan: OTC-19311, 8 p.

Schoderbek, D., Martin, K., Howard, J., Silpngarmlert, S., Hester, K., 2012. North Slope hydrate field trial: CO2/CH4 exchange. In: Proceedings Arctic Technology Conference, December 3-5, 2012, Houston, Texas, 17 p.

Sethi, A.K., Sathe, A.V., Ramana, M.V., 2004. Potential natural gas hydrate resources in Indian offshore areas. In: Proceedings of the American Association of Petroleum Geologists Hedberg Research Conference on Natural Gas Hydrates. Energy Resources Potential and Associated Geologic Hazards, Vancouver, British Columbia, Canada. September 12-16, 2004, 6 p.

Schultheiss, P.J., Holland, M., Humphrey, G., 2009. Wireline coring and analysis under pressure: recent use and future developments of the HYACINTH system. Sci. Drill. 7, 44-50.

Shankar, U., Riedel, M., 2011. Gas hydrate saturation in the Krishna-Godavari Basin from p-wave velocity and electrical resistivity logs. J. Mar. Pet. Geol. 28 (10), $1768-1778$.

Shankar, U., Riedel, M., 2014. Assessment of gas hydrate saturation in marine sediments from resistivity and compressional-wave velocity log measurements in the Mahanadi Basin, India. JMPG-D-13-00214 J. Mar. Pet. Geol. (in this issue).

Shelander, D., Dai, J., Bunge, G., 2010. Predicting saturation of gas hydrates using pre-stack seismic data, Gulf of Mexico. J. Mar. Geophys. 31 (1-2), 39-57.

Shelander, D., Dai, J., Bunge, G., Singh, S., Eissa, M., Fisher, K., 2012. Estimating saturation of Gas hydrates using conventional 3D seismic data, Gulf of Mexico Joint Industry project leg II. J. Mar. Pet. Geol. 34, 96-110.

Shipley, T., Houston, M., Buffler, R., Shaub, F., McMillen, K., Ladd, J., Worze, J., 1979. Seismic evidence for widespread possible gas hydrate horizons on continental slopes and rises. Am. Assoc. Pet. Geol. Bull. 63, 2204-2213.

Torres, M.E., Tréhu, A.M., Cespedes, N., Kastner, M., Wortmann, U.G., Kim, J.H., Long, P., Malinverno, A., Pohlman, J.W., Riedel, M., Collett, T.S., 2008. Methane hydrate formation in turbidite sediments of northern Cascadia, IODP Expedition 311. Earth Planet. Sci. Lett. 271, 170-180.

Tréhu, A.M., Bohrmann, G., Rack, F.R., Torres, M.E., et al., 2004. Volume 204 initial reports, drilling Gas hydrates on hydrate Ridge, Cascadia Continental margin. In: Proceedings of the Ocean Drilling Program, vol. 204. Ocean Drilling Program. http://www-odp.tamu.edu/publications/204_IR/front.htm.

Tsuji, Y., Fujii, T., Hayashi, M., Kitamura, R., Nakamizu, M., Ohbi, K., Saeki, T., Yamamoto, K., Namikawa, T., Inamori, T., Oikawa, N., Shimizu, S., Kawasaki, M., Nagakubo, S., Matsushima, J., Ochiai, K., Okui, T., 2009. Methane-hydrate occurrence and distribution in the eastern Nankai trough, Japan: findings of the Tokai-oki to Kumano-nada methane-hydrate drilling program. Natural Gas Hydrates - A Review, in Collett, T., Johnson, A., Knapp, C., Boswell, R., eds. In: Collett, T., Johnson, A., Knapp, C., Boswell, R. (Eds.), Natural Gas Hydrates Energy Resource Potential and Associated Geologic Hazards, American Association of Petroleum Geologists Memoir 89, pp. 228-246.

Varma, B.K., 2004. Studies in Gas Hydrate - ONGC Approach (unpublished report). KDMIPE, Oil and Natural Gas Commission, Dehradun, India, 3 p.

Wegner, S.A., Campbell, K.J., 2014. Drilling hazard assessment for hydrate bearing sediments including drilling through the BSRs. J. Mar. Pet. Geol. (in this issue).

Yamamoto, K., Terao, Y., Fujii, T., Terumichi, I., Seki, M., Matsuzawa, M., Kanno, T., 2014. Operational overview of the first offshore production test of methane hydrates in the Eastern Nankai Trough. In: Proceeding of the Offshore Technology Conference, OTC-25243, Houston, Texas, 15 p. 